AEI-2011-049

\title{
ELLIPTIC HYPERGEOMETRY OF SUPERSYMMETRIC DUALITIES II. ORTHOGONAL GROUPS, KNOTS, AND VORTICES
}

\author{
V. P. SPIRIDONOV AND G. S. VARTANOV
}

\begin{abstract}
We consider Seiberg electric-magnetic dualities for $4 d \mathcal{N}=1 \mathrm{SYM}$ theories with $S O(N)$ gauge group. For all such known theories we construct superconformal indices (SCIs) in terms of elliptic hypergeometric integrals. Equalities of these indices for dual theories lead both to proven earlier special function identities and new conjectural relations for integrals. In particular, we describe a number of new elliptic beta integrals associated with the $s$-confining theories with the spinor matter fields. Reductions of some dualities from $S P(2 N)$ to $S O(2 N)$ or $S O(2 N+1)$ gauge groups are described. Interrelation of SCIs and the Witten anomaly is briefly discussed. Possible applications of the elliptic hypergeometric integrals to a twoparameter deformation of $2 d$ conformal field theory and related matrix models are indicated. Connections of the reduced SCIs with the state integrals of the knot theory, generalized AGT duality for $(3+3) d$ theories, and a $2 d$ vortex partition function are described.
\end{abstract}

Dedicated to D.I. Kazakov on the occasion of his 60th birthday

\section{CONTENTS}

1. Introduction

2. Reduction of $\mathcal{N}=1$ dualities from symplectic to orthogonal gauge groups

2.1. Dualities without spinor matter

2.2. Connection to the Witten anomaly

2.3. $S O / S P$ gauge group theories with small number of flavors

3. $S$-confining theories with the spinor matter

3.1. Confinement for $S O(7)$ gauge group

3.2. $G=S O(8)$

3.3. $G=S O(9)$

3.4. $G=S O(10)$

3.5. $G=S O(11)$

3.6. $G=S O(12)$

3.7. $G=S O(13)$

3.8. $G=S O(14)$

4. Self-dual theories with the spinor matter

5. Seiberg dualities for $S O(N)$ gauge group with the spinor matter

5.1. $G=S O(5)$ and $F=S U\left(N_{f}\right) \times S O(4) \times U(1)$

5.2. $S O(7)$ gauge group with $N_{f}$ fundamentals

5.3. $G=S O(7)$ and $F=S U\left(N_{f}\right) \times U(1)$

5.4. $G=S O(7)$ and $F=S U\left(N_{f}\right) \times S U(2) \times U(1)$

5.5. $G=S O(8)$ and $F=S U\left(N_{f}\right) \times U(1)$

5.6. $G=S O(8)$ and $F=S U\left(N_{f}\right) \times U(1)_{1} \times U(1)_{2}$

5.7. $G=S O(9)$ and $F=S U\left(N_{f}\right) \times U(1)$

5.8. $G=S O(10)$ and $F=S U\left(N_{f}\right) \times U(1)$

6. Matrix models and an elliptic deformation of $2 d$ CFT 
6.1. Elliptic Selberg integral 40

6.2. $q$-Selberg integral $\quad 40$

6.3. Reduction to the Selberg integral $\quad 41$

6.4. A higher order elliptic Selberg integral 42

7. Connection to the knot theory $\quad 42$

7.1. The figure-eight knot $\quad 43$

7.2. The trefoil knot $\quad 46$

7.3. Some other integrals $\quad 47$

8. Reduction to the $2 d$ vortex partition function 48

9. Conclusion $\quad 50$

\begin{tabular}{ll|} 
Appendix A. Characters of representations of orthogonal groups & 52 \\
\hline
\end{tabular}

References

\section{INTRODUCTION}

Gauge field theories play a crucial role in the modern theory of elementary particles. A generalization of the notion of electric-magnetic duality from electrodynamics to non-abelian gauge theories was suggested in the fundamental work of Goddard, Nuyts, and Olive [54]. In the asymptotically free theories the spectrum of elementary excitations in the high energy region is found from the free lagrangian. In the infrared region the interaction becomes strong and one has to pass to the description in terms of collective degrees of freedom (in the usual quantum chromodynamics one should describe formation of the hadrons out of quarks and gluons). The electric-magnetic duality relates these two energy scales and is also referred to as the strongweak coupling duality transformation. To the present moment consistent consideration of such transformations in $4 d$ space-time has been given only in the maximally extended $\mathcal{N}=4$ [91, $\mathcal{N}=2$ [106], and $\mathcal{N}=1$ [103, 104] supersymmetric field theories. In comparison to the dualities for $\mathcal{N}>1$ there exists a whole zoo of different Seiberg dualities for $\mathcal{N}=1$ SYM theories (see, e.g., surveys [67, 108]). The problem of their classification using some group-theoretical approach is still open. For a survey of the current status of development of supersymmetric gauge theories, see [105].

Highly nontrivial generalizations of the Witten index called superconformal indices (SCIs) were proposed recently by Kinney et al [76] and Römelsberger [99, 100]. SCIs count BPS states protected by one supercharge and its (superconformal) conjugate which cannot be combined to form long multiplets. They can be considered as twisted partition functions in the Hilbert space of BPS states which are determined by specific matrix integrals over the classical Lie groups. SCI is a conformal manifold invariant [53] which does not change under the marginal deformations [100, 119.

In this paper we continue a systematic study of electric-magnetic dualities for $\mathcal{N}=1 \mathrm{SYM}$ theories and $s$-confining theories initiated in [116]. We use for that the theory of elliptic hypergeometric integrals (EHIs) developed by the first author in [110, 111, 112]. The crucial observation on the coincidence of SCIs with such integrals was done by Dolan and Osborn in [36. In a sequel of papers [115, 116, 117, 118, 119, 120, 123] we analyzed known supersymmetric dualities, described deep relations between them and the properties of EHIs, and, using these relations, discovered many new dualities. Related questions were considered also in [46, 47].

SCI techniques provides currently the most rigorous mathematical justification of $\mathcal{N}=1$ supersymmetric dualities [103, 104], and it serves as a very powerful tool for getting new 
insights. For instance, it has led to $\mathcal{N}=1$ dualities lying outside the conformal window [118, it is useful for consideration of the AdS/CFT correspondence for gauge groups of infinite [76, 83, 84] and finite [85, 119] rank. It can be applied to theories which are difficult to treat by usual physical tools [123. Another interesting fact is that $4 d$ SCIs can be reduced to $3 d$ partition functions [39, 56, 68, 71] yielding 3d dualities [37, 49, 62]. Recently in [86] SCIs with the half-BPS superconformal surface operator have been studied. EHIs are connected with the relativistic Calogero-Sutherland type models where they describe either special wave functions or the normalizations of particular wave functions [113]. In [117] such a connection was conjectured to extend to all SCIs. EHIs provide a unification of known solvable models of statistical mechanics on $2 d$ lattices [8, 115]. In [115] it was shown that SCIs of the simple gauge group SYM theories have the meaning of partition functions of elementary cells of $2 d$ integrable lattice models, and corresponding full partition functions describe SCIs of particular quiver theories. In this picture, the Seiberg duality has the meaning of a generalized KramersWannier duality transformation for partition functions. As shown in $[120], S L(3, \mathbb{Z})$-modular transformation properties of EHIs are responsible for 't Hooft anomaly matching conditions in dual theories.

SCI techniques applies not only to $4 d$ field theories, but also to $3 d$ models [7, 63, 75, 78]. In [78. the equality of SCIs of some $3 d$ dual theories with $U(1)$ gauge group was proved rigorously for $N_{f}=1,2$ flavors, and in [70] this result was generalized to arbitrary $N_{f}$. The analytical proof of the coincidence for partition functions of some $3 d$ quiver $\mathcal{N}=4$ mirror symmetric theories was considered in [10].

There are several different ways of computing SCIs. The localization method was used by Moore, Nekrasov and Shatashvili for computing the principal contribution to the Witten index of supersymmetric theories expressed as some contour integrals over $S U(N)$ group [82]. Later this approach was generalized by Nekrasov and Shadchin [90] for solving $\mathcal{N}=2$ supersymmetric field theories with symplectic and orthogonal gauge groups. For $\mathcal{N}=1 \mathrm{SYM}$ theories Römelsberger [99, 100] derived SCIs using the operator approach to free superconformal field theories (SCFTs) and suggested that SCIs for Seiberg dual theories coincide. For the asymptotically free theories in the ultraviolet region this is formally justified. In [76], Kinney et al derived SCI for $\mathcal{N}=4 U(N)$-SYM theory using the representation theory for free SCFTs [34] and targeting mostly the AdS/CFT correspondence. SCIs for extended superconformal field theories can be derived directly from the partition functions by imposing some restriction on the parameters [12]. In [87], the localization technique was used for derivation of SCI for $\mathcal{N}=4$ SYM theory. In [88, 89, 92], this method was used for computing partition functions of $\mathcal{N}=2$ SYM theories. For related questions concerning counting the BPS operators, see also [9, 35, 44]. One can get $\mathcal{N}=2$ and $\mathcal{N}=4 \mathrm{SYM}$ theories out of $\mathcal{N}=1$ theories by adjusting the matter fields content and superpotentials. Analogously, SCIs of extended theories can be obtained from $\mathcal{N}=1$ SCIs by appropriate fitting of the set of representations [117].

In this paper we are investigating SCIs for $4 d \mathcal{N}=1$ theories with orthogonal gauge groups. The most interesting $S O(N)$-dualities arise from the matter fields in spinor representation. Dualities without such matter fields can be obtained by reductions from the $S P(2 N)$-gauge group cases. Additionally, we outline possible application of some of EHIs (particular 4d SCIs) to a hypothetical elliptic deformation of $2 d$ CFT. As an important relation between $4 d$ and $3 d$ field theories, we show that reductions of $4 d$ SCIs to the hyperbolic $q$-hypergeometric level yield the state integrals of knots [28, 30, 32, 58, 59]. Further reduction of a particular hyperbolic $q$-hypergeometric integral emerging in this way is shown to give a $2 d$ vortex partition function. 
By definition SCIs count gauge invariant operators which saturate the BPS bounds for short and semi-short multiplets. $\mathcal{N}=1$ SCFTs are based on the $S U(2,2 \mid 1)$ space-time symmetry group which is generated by the following set of operators: $J_{i}, \bar{J}_{i}$ - the generators of two $S U(2)$ subgroups forming the $4 d$ Lorentz group $S O(3,1)$, translations, $P_{\mu}, \mu=1,2,3$, 4 , special conformal transformations, $K_{\mu}$, the dilations, $H$, and also the $U(1)_{R}$-group generator $R$. Apart from these bosonic generators there are supercharges $Q_{\alpha}, \bar{Q}_{\dot{\alpha}}$ with $\alpha, \dot{\alpha}=1,2$ and their superconformal partners $S_{\alpha}, \bar{S}_{\dot{\alpha}}$. The full set of commutation relations for these operators can be found, for instance, in [117. Taking a distinguished pair of intrinsically superconformal charges [99], for example, $Q=\bar{Q}_{1}$ and $Q^{\dagger}=-\bar{S}_{1}$, one has

$$
\left\{Q, Q^{\dagger}\right\}=2 \mathcal{H}, \quad Q^{2}=\left(Q^{\dagger}\right)^{2}=0, \quad \mathcal{H}=H-2 \bar{J}_{3}-3 R / 2 .
$$

In this case the superconformal index is defined by the matrix integral

$$
I\left(p, q, f_{k}\right)=\int_{G} d \mu(g) \operatorname{Tr}\left((-1)^{\mathcal{F}} p^{\mathcal{R} / 2+J_{3}} q^{\mathcal{R} / 2-J_{3}} e^{\sum_{a} g_{a} G^{a}} e^{\sum_{k} f_{k} F^{k}} e^{-\beta \mathcal{H}}\right), \quad \mathcal{R}=R+2 \bar{J}_{3},
$$

where $\mathcal{F}$ is the fermion number operator and $d \mu(g)$ is the invariant measure of the gauge group $G$. We explicitly singled out the integration over gauge group, though most often it is assumed to be a part of the gauge invariant trace. To calculate the index one should not consider the whole space of states, but only zero modes of the operator $\mathcal{H}$ because contributions of states not annihilated by the supercharge $Q$ cancel each other. The chemical potentials $g_{a}, f_{k}$ correspond to the gauge $G$ and flavor $F$ symmetry group generators $G^{a}$ and $F^{k}$, respectively.

$4 d$ SCI coincides with the supersymmetric index on $S^{3} \times S^{1}$ manifold. For a latest discussion of such space-time manifestations, see [45, 105]. According to the Römelsberger prescription (in the form suggested in [36]) one should first compute the single particle index, given by the following general formula

$$
\begin{aligned}
\operatorname{ind}(p, q, z, y) & =\frac{2 p q-p-q}{(1-p)(1-q)} \chi_{a d j}(z) \\
& +\sum_{j} \frac{(p q)^{r_{j}} \chi_{R_{F}, j}(y) \chi_{R_{G}, j}(z)-(p q)^{1-r_{j}} \chi_{\bar{R}_{F}, j}(y) \chi_{\bar{R}_{G}, j}(z)}{(1-p)(1-q)}
\end{aligned}
$$

Here the first term describes the contribution of gauge fields belonging to the adjoint representation of group $G$; the sum over $j$ corresponds to the chiral matter superfields $\Phi_{j}$ transforming as the gauge group representations $R_{G, j}$ and the flavor symmetry group representations $R_{F, j}$ with $2 r_{j}$ being their $R$-charges. The functions $\chi_{a d j}(z), \chi_{R_{F}, j}(y)$ and $\chi_{R_{G}, j}(z)$ are the characters of representations with $z$ and $y$ being the maximal torus variables of $G$ and $F$ groups, respectively. All the characters needed for this work are explicitly listed in Appendix A. Originally [36, 100. the index was expressed in terms of variables $x, t$ related to our bases as $p=t x, q=t x^{-1}$. We remark that as a result of the change of variables there appears a sign ambiguity, the term $(p q)^{r_{j}}$ in $(1.3)$ can be written as $( \pm \sqrt{p q})^{R_{j}}$, where $R_{j}$ are $R$-charges of the fields, and this may influence the balancing condition for integrals below.

To obtain the full superconformal index, the single particle states index (1.3) is inserted into the "plethystic" exponential which is then averaged over the gauge group:

$$
I(p, q, y)=\int_{G} d \mu(z) \exp \left(\sum_{n=1}^{\infty} \frac{1}{n} \operatorname{ind}\left(p^{n}, q^{n}, z^{n}, y^{n}\right)\right) .
$$

It appears that such matrix integrals are expressed in terms of the new special functions of mathematical physics known as elliptic hypergeometric integrals which were discovered in 
[110, 111, 112] (see also [114] for a general survey). Their simplest representative - the exactly computable elliptic beta integral [110] is the top level known generalization of the Euler beta integral, the Askey-Wilson and Rahman $q$-beta integrals [3]. As found in [36], it describes the confinement phenomenon for $4 d \mathcal{N}=1 \mathrm{SYM}$ theory with $S U(2)$ gauge group and 6 quarks which is dual to the theory of free baryons forming the absolutely antisymmetric tensor representation of the flavor group $S U(6)$. On the base of a very large number of explicit examples listed in [117], we conjectured that to every supersymmetric duality there corresponds either an exact integration formula for elliptic beta integrals or a nontrivial Weyl group symmetry transformation for the higher order EHIs .

One important remark is in order. Described index computation algorithm does not impose in advance any constraint on the fugacities, whereas the EHI identities used for establishing equalities of SCIs require neat fitting of parameter constraints for their existence (see below). It would be interesting to find the arguments leading to needed constraints for fugacities directly in formulas (1.3) and (1.4).

This paper can be considered as a second part of the work [117] since we cover several subjects skipped in it. However, there are still some interesting questions touched in [117], but not included in this paper. In particular, we do not discuss SCIs of quiver theories which have attracted recently some interest in [21, 115].

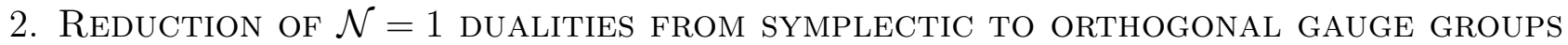

2.1. Dualities without spinor matter. Let us show that known $\mathcal{N}=1$ dualities with $S O(n)$ gauge group without matter in the spinor representation can be derived as consequences of known $S P(2 N)$ gauge group dualities. At the level of SCIs this implication is achieved by particular restriction of the values of a number of parameters in the corresponding EHIs, as observed first by Dolan and Osborn for the simplest cases [36]. In the present section we discuss such reductions in more detail. The spinor matter theories will be considered later on.

We start from $\mathcal{N}=1 \mathrm{SYM}$ theory with $S P(2 N)$ gauge group and $2 N_{f}$ quarks in the fundamental representation having the global symmetry group $S U\left(2 N_{f}\right) \times U(1)_{R}$. The matter fields are described in the table below, where we indicate their representation types for the gauge and flavor groups and provide $R$-charges

\begin{tabular}{|l|c|c|c|}
\hline & $S P(2 N)$ & $S U\left(2 N_{f}\right)$ & $U(1)_{R}$ \\
\hline$Q$ & $f$ & $f$ & $1-(N+1) / N_{f}$ \\
\hline
\end{tabular}

In this and all other tables below we skip the vector superfield $V$ (or its dual partner $\widetilde{V}$, which is absent in confining theories) described by the adjoint representation of $G$ and singlets of the non-abelian part of the flavor group, and having trivial hypercharges for the abelian global groups.

The dual magnetic theory constructed by Intriligator and Pouliot 65] has the same flavor group and the gauge group $G=S P(2 \widetilde{N})$, where $\widetilde{N}=N_{f}-N-2$, with the matter field content described in the table below

\begin{tabular}{|c|c|c|c|}
\hline & $S P(2 \widetilde{N})$ & $S U\left(2 N_{f}\right)$ & $U(1)_{R}$ \\
\hline$q$ & $f$ & $\bar{f}$ & $(N+1) / N_{f}$ \\
$M$ & 1 & $T_{A}$ & $2(\widetilde{N}+1) / N_{f}$ \\
\hline
\end{tabular}

where $f(\bar{f})$ denotes (anti)fundamental representation and $T_{A}$ denotes the antisymmetric tensor of the second rank. 
The conformal window for this duality is $3(N+1) / 2<N_{f}<3(N+1)$; it emerges from the demand that both dual theories are asymptotically free in the one-loop approximation. The Seiberg electric-magnetic duality at the infrared fixed points of these theories, which is not proven rigorously yet, had the following justifying arguments [104]:

- the 't Hooft anomaly matching conditions are satisfied. They were shown in [120] to follow from the $S L(3, \mathbb{Z})$-group transformation properties of EHIs;

- matching reduction of the number of flavors $2 N_{f} \rightarrow 2\left(N_{f}-1\right)$. Integrating out $2 N_{f},\left(2 N_{f}-\right.$ 1)-th flavor quarks by adding the mass term in electric theory results in Higgsing the magnetic theory gauge group with decoupling of a number of meson fields. For SCIs this is realized by restricting a pair of parameters, $t_{2 N_{f}} t_{2 N_{f}-1}=p q$ [116, 117];

- matching of the moduli spaces and gauge invariant operators in dual theories. This information is believed to be hidden in the topological meaning of SCIs.

We need the following EHI on the $B C_{n}$ root system

$$
I_{n}^{(m)}(\underline{t} ; p, q)=\frac{(p ; p)_{\infty}^{n}(q ; q)_{\infty}^{n}}{2^{n} n !} \int_{\mathbb{T}^{n}} \prod_{1 \leq i<j \leq n} \frac{1}{\Gamma\left(z_{i}^{ \pm 1} z_{j}^{ \pm 1}\right)} \prod_{j=1}^{n} \frac{\prod_{i=1}^{2(m+n+2)} \Gamma\left(t_{i} z_{j}^{ \pm 1}\right)}{\Gamma\left(z_{j}^{ \pm 2}\right)} \prod_{j=1}^{n} \frac{d z_{j}}{2 \pi \mathrm{i} z_{j}}
$$

where $\mathbb{T}$ is the unit circle with positive orientation, all $\left|t_{i}\right|<1, \prod_{i=1}^{2(m+n+2)} t_{i}=(p q)^{m+1}$,

$$
(z ; q)_{\infty}=\prod_{i=0}^{\infty}\left(1-z q^{i}\right), \quad|q|<1
$$

is the standard infinite $q$-shifted factorial [3] and

$$
\Gamma(z) \equiv \Gamma(z ; p, q)=\prod_{i, j=0}^{\infty} \frac{1-z^{-1} p^{i+1} q^{j+1}}{1-z p^{i} q^{j}}, \quad|p|,|q|<1
$$

is the elliptic gamma function [114]. We use the convention

$$
\Gamma\left(t_{1}, \ldots, t_{k}\right)=\Gamma\left(t_{1}\right) \ldots \Gamma\left(t_{k}\right), \quad \Gamma\left(t z^{ \pm 1}\right)=\Gamma(t z) \Gamma\left(t z^{-1}\right) .
$$

Then the algorithm for construction SCIs described above yields for the electric theory $I_{E}^{S P(2 N)}=$ $I_{N}^{\left(N_{f}-N-2\right)}\left(t_{1}, \ldots, t_{2 N_{f}} ; p, q\right)$ [36, 117]. The dual magnetic theory has SCI of the form

$$
I_{M}^{S P(2 \widetilde{N})}=\prod_{1 \leq i<j \leq 2 N_{f}} \Gamma\left(t_{i} t_{j}\right) I_{N_{f}-N-2}^{(N)}\left((p q)^{1 / 2} / t_{1}, \ldots,(p q)^{1 / 2} / t_{2 N_{f}} ; p, q\right) .
$$

Römelsberger's conjecture on the equality of SCIs for dual theories $I_{E}^{S P(2 N)}=I_{M}^{S P(2 \widetilde{N})}$ was proven in [36] on the basis of the symmetry transformation for integrals established in [97]. For $N=1$ the full symmetry group of SCI is $W\left(E_{7}\right)$. The key transformation generating this group was found earlier in [111]. Its physical consequences for multiple dualities have been studied in [116] and the superpotentials for such theories were investigated later in [74]. Altogether the results of [36, 116, 117] gave a new powerful, most rigorous from the mathematical point of view confirmation of the Seiberg duality, complementing the tests mentioned above.

It should be stressed that this and all other equalities of SCIs of dual theories are true or supposed to be true only if the values of parameters in all integrals guarantee that only sequences of poles of the integrands converging to zero are located inside the contour of integration $\mathbb{T}$ (otherwise one should use the nontrivial analytical continuation procedure for identities to be true in other regions of parameters). 
Consider now the Seiberg duality for $\mathcal{N}=1$ SYM theories with orthogonal gauge group [104]. The electric theory matter fields are described in the following table

\begin{tabular}{|c|c|c|c|c|}
\hline & $S O(N)$ & $S U\left(N_{f}\right)$ & $Z_{2 N_{f}}$ & $U(1)_{R}$ \\
\hline$Q$ & $f$ & $f$ & $k$ & $\frac{N_{f}-N+2}{N_{f}}$ \\
\hline
\end{tabular}

and for the magnetic theory one has

\begin{tabular}{|c|c|c|c|c|}
\hline & $S O(\widetilde{N})$ & $S U\left(N_{f}\right)$ & $Z_{2 N_{f}}$ & $U(1)_{R}$ \\
\hline$q$ & $f$ & $\bar{f}$ & $-k$ & $\frac{N-2}{N_{f}}$ \\
$M$ & 1 & $T_{S}$ & $2 k$ & $2 \frac{N_{f}-N+2}{N_{f}}$ \\
\hline
\end{tabular}

where $T_{S}$ denotes the absolutely symmetric tensor of second rank and $\widetilde{N}=N_{f}-N+4$. The conformal window [104] for this duality has the form $3(N-2) / 2<N_{f}<3(N-2)$, which guarantees existence of the non-trivial infrared fixed points (one should be careful with the use of such windows since there are examples [118] of dualities lying outside them).

In these tables we explicitly indicated existence of the discrete $Z_{2 N_{f}}$ symmetry [104, 66]. In order to take it into account in the construction of SCIs we modify the Römelsberger prescription for orthogonal groups. Introduce the single particles states index

$$
\begin{aligned}
\operatorname{ind}\left(p, q, z, y, x_{k}\right) & =\frac{2 p q-p-q}{(1-p)(1-q)} \chi_{a d j}(z) \\
& +\sum_{j} \frac{x_{k}(p q)^{r_{j}} \chi_{R_{F}, j}(y) \chi_{R_{G}, j}(z)-(p q)^{1-r_{j}} \chi_{\bar{R}_{F}, j}(y) \chi_{\bar{R}_{G, j}}(z) / x_{k}}{(1-p)(1-q)}
\end{aligned}
$$

where $x_{k}=e^{\pi \mathrm{i} k / N_{f}}, k=0, \ldots, 2 N_{f}-1$, and apply the general formula (1.4) with the powers $x_{k}^{n}$ in the plethystic exponential.

Orthogonal groups $S O(n)$ are qualitatively different for even $n=2 N$ (root system $D_{N}$ ) and odd $n=2 N+1$ (roots system $B_{N}$ ). SCIs in the electric theory take the form

$$
I_{E}^{S O(2 N)}=\frac{(p ; p)_{\infty}^{N}(q ; q)_{\infty}^{N}}{2^{N-1} N !} \int_{\mathbb{T}^{N}} \frac{\prod_{i=1}^{N_{f}} \prod_{j=1}^{N} \Gamma\left(t_{i} z_{j}^{ \pm 1}\right)}{\prod_{1 \leq i<j \leq N} \Gamma\left(z_{i}^{ \pm 1} z_{j}^{ \pm 1}\right)} \prod_{j=1}^{N} \frac{d z_{j}}{2 \pi \mathrm{i} z_{j}}
$$

where the balancing condition reads $\prod_{i=1}^{N_{f}} t_{i}= \pm(p q)^{N_{f} / 2-N+1}$, and

$$
I_{E}^{S O(2 N+1)}=\frac{(p ; p)_{\infty}^{N}(q ; q)_{\infty}^{N}}{2^{N} N !} \prod_{i=1}^{N_{f}} \Gamma\left(t_{i}\right) \int_{\mathbb{T}^{N}} \frac{\prod_{i=1}^{N_{f}} \prod_{j=1}^{N} \Gamma\left(t_{i} z_{j}^{ \pm 1}\right)}{\prod_{j=1}^{N} \Gamma\left(z_{j}^{ \pm 1}\right) \prod_{1 \leq i<j \leq N} \Gamma\left(z_{i}^{ \pm 1} z_{j}^{ \pm 1}\right)} \prod_{j=1}^{N} \frac{d z_{j}}{2 \pi \mathrm{i} z_{j}}
$$

where the balancing condition is $\prod_{i=1}^{N_{f}} t_{i}= \pm(p q)^{N_{f} / 2-N+1 / 2}$. Here $t_{i}:=x_{k}(p q)^{r_{i}} y_{i}$ and the effect of the discrete chemical potential $k$ is reduced to the sign value on the right-hand side of the balancing condition.

The magnetic theory SCI can be written in the form:

$$
I_{M}(\underline{t} ; p, q)^{S O(\widetilde{N})}=\prod_{1 \leq i<j \leq N_{f}} \Gamma\left(t_{i} t_{j}\right) \prod_{i=1}^{N_{f}} \Gamma\left(t_{i}^{2}\right) I_{E}\left(\frac{\sqrt{p q}}{\underline{t}} ; p, q\right)^{S O(\widetilde{N})}
$$


To show the duality relation $I_{E}(\underline{t} ; p, q)^{S O(N)}=I_{M}(\underline{t} ; p, q)^{S O(\widetilde{N})}$ one has to restrict parameters in the $S P(2 N)$-indices [36]. First we identify

$$
I_{E}(\underline{t} ; p, q)^{S O(2 n)}= \begin{cases}2 I_{n}^{\left(\frac{1}{2}\left(N_{f}+4\right)-n\right)}(\underline{t}, \underline{u} ; p, q), & N_{f} \text { even } \\ 2 I_{n}^{\left(\frac{1}{2}\left(N_{f}+3\right)-n\right)}(\underline{t}, \underline{v} ; p, q), & N_{f} \text { odd }\end{cases}
$$

where parameters $\underline{u}$ and $\underline{v}$ in $I_{n}^{(m)}$ are chosen as

$$
\underline{u}=\{ \pm 1, \pm \sqrt{p}, \pm \sqrt{q}, \pm \sqrt{p q}\}, \quad \underline{v}=\{ \pm 1, \pm \sqrt{p}, \pm \sqrt{q},-\sqrt{p q}\} .
$$

Analogously,

$$
I_{E}(\underline{t} ; p, q)^{S O(2 n+1)}= \begin{cases}\prod_{i=1}^{N_{f}} \Gamma\left(t_{i}\right) I_{n}^{\left(\frac{1}{2}\left(N_{f}+2\right)-n\right)}\left(\underline{t}, \underline{u}^{\prime}\right), & N_{f} \text { even } \\ \prod_{i=1}^{N_{f}} \Gamma\left(t_{i}\right) I_{n}^{\left(\frac{1}{2}\left(N_{f}+3\right)-n\right)}\left(\underline{t}, \underline{v}^{\prime} ; p, q\right), & N_{f} \text { odd }\end{cases}
$$

where

$$
\underline{u}^{\prime}=\{-1, \pm \sqrt{p}, \pm \sqrt{q},-\sqrt{p q}\}, \quad \underline{v}^{\prime}=\{-1, \pm \sqrt{p}, \pm \sqrt{q}, \pm \sqrt{p q}\} .
$$

These relations are based on the duplication formula for the elliptic gamma function

$$
\Gamma\left(z^{2}\right)=\prod_{\varepsilon= \pm 1} \Gamma(\varepsilon z, \varepsilon \sqrt{p} z, \varepsilon \sqrt{q} z, \varepsilon \sqrt{p q} z)
$$

and the inversion formula $\Gamma(z) \Gamma(p q / z)=1$. They allow one to reduce EHIs from $S P(2 n)$-group to $S O(2 n)$ or $S O(2 n+1)$ and, simultaneously, reduces mesons from $T_{A^{-}}$to $T_{S^{-}}$-representation.

The same line of arguments works for checking equality of SCIs for many other known dualities of orthogonal gauge group theories whose matter content we list below:

- the antisymmetric tensor of the second rank (or the adjoint representation) and quarks in the fundamental representation, see [79] for the duality between interacting field theories and [22, 77] for the $s$-confining theory;

- the symmetric tensor of the second rank and quarks in the fundamental representation, see [64] for nontrivial dual gauge group case and [22, 77] for the $s$-confining theory;

- two matter fields - symmetric tensors of the second rank and quarks in the fundamental representation, see [14, 77];

- one matter field - the symmetric tensor of the second rank, and another field, the antisymmetric tensor of the second rank, together with the quarks in the fundamental representation, see [14, 77].

For brevity we are not presenting explicitly SCIs of these theories and do not indicate how they are related to $S P(2 N)$-group indices considered in [117] since they are easily obtained by reductions similar to the one described above. Moreover, one can obtain new orthogonal gauge group dualities with the flavor group composed of several $S P(2 m)$-groups and $S U(4)$ group after a similar reduction of the duality considered in Sect. 7 of [117] (as well as the related $s$-confining theory). The general question why $S O$-dualities for theories without spinor matter can be derived from $S P$-theories is not understood from the physical point of view yet.

Now we would like to discuss some special cases in more detail. Consider $G=S O(n)$ theory with $N_{f}=n-1$ quarks known to have three dual pictures [66]: electric, magnetic, and dyonic. For $G=S O(2 N+1)$ with $2 N$ quarks SCI is obtained from (2.5) with $N_{f}$ replaced by $2 N$. The magnetic dual has $S O(3)$ gauge group with SCI

$$
I_{M}^{S O(3)}=\prod_{1 \leq m<s \leq 2 N} \Gamma\left(t_{m} t_{s}\right) \prod_{i=1}^{2 N} \Gamma\left(t_{i}^{2}, \frac{\sqrt{p q}}{t_{i}}\right) \frac{(p ; p)_{\infty}(q ; q)_{\infty}}{2} \int_{\mathbb{T}} \frac{\prod_{i=1}^{2 N} \Gamma\left(\frac{\sqrt{p q}}{t_{i}} y^{ \pm 1}\right)}{\Gamma\left(y^{ \pm 1}\right)} \frac{d y}{2 \pi \mathrm{i} y}
$$


the balancing condition here reads $\prod_{m=1}^{2 N} t_{m}=\sqrt{p q}$. These expressions can also be obtained from $S P(2 N)$-indices with $N_{f}=N+3$. The moduli space of vacua of the $S O(3)$-theory has two non-trivial points leading to two dual theories. One of them is the original $S O(2 N+1)$ electric theory, and the second one is the $S O(2 N+1)$-dyonic theory, which is obtained from the electric one by adding a particular term to the superpotential and shifting the theta angle by $\pi$. The electric and dyonic theories are related to each other by the "weak-to-weak" $T$-duality transformation and, therefore, their superconformal indices are identical, $I_{D} \equiv I_{E}$. These duality transformations form the permutation group $S_{3}$, a subgroup of the $S L(2, \mathbb{Z})$-group, interchanging the three different theories.

The same arguments apply to $\mathcal{N}=1 \mathrm{SYM}$ theory with $S O(2 N)$ gauge group and $2 N-1$ quarks. Restricting seven parameters in $I_{E}^{S P(2 N)}$ (with $N_{f}=N+3$ ) as $1, \pm \sqrt{p}, \pm \sqrt{q}, \pm \sqrt{p q}$, one obtains SCI of the electric theory identically coinciding with the index of the dyonic theory. Substituting the same constraints to $I_{M}^{S P(2 N)}$ one obtains SCI of the $S O(3)$-magnetic theory. In both cases the balancing condition reads $\prod_{i=1}^{2 N-1} t_{i}=1$, i.e. at least one of the parameters $t_{i}$ has modulus greater than 1 , which requires an appropriate deformation of the integration contours for separation of relevant sequences of integrand poles.

As to the self-dual case of $S O(3)$-gauge group, its SCIs $I_{E}^{S O(3)}$ and $I_{M}^{S O(3)}$ depend on two parameters with the balancing condition $t_{1} t_{2}=\sqrt{p q}$. Remarkably, after taking into account the latter constraint, the index $I_{M}^{S O(3)}$ becomes identically equal to $I_{E}^{S O(3)}$. So, the electric, magnetic, and dyonic theories differ from each other only by particular terms in the superpotential (governed by the parameter $e=0, \pm 1$ in [66]) and have SCIs of identical shape.

According to Seiberg [104], the case $G=S O(n)$ with $N_{f}=n-2$ has the dual gauge group $S O(2)$, i.e. the magnetic theory coincides with $\mathcal{N}=1$ abelian theory describing the supersymmetric photon with the gauge group $U(1)$. This duality can be deduced from the $S P(2 N) \leftrightarrow S P\left(2\left(N_{f}-N-2\right)\right)$ duality with $N_{f}=N+3$. Corresponding SCIs are obtained by imposing appropriate constraints on the parameters, as described above. For $G=S O(2 N+1)$ SCI is given by expression (2.5) with $N_{f}$ replaced by $2 N-1$. The dual SCI has the form

$$
I_{M}^{S O(2)}=\prod_{1 \leq m<s \leq 2 N-1} \Gamma\left(t_{m} t_{s}\right) \prod_{i=1}^{2 N-1} \Gamma\left(t_{i}^{2}\right) \frac{(p ; p)_{\infty}(q ; q)_{\infty}}{2} \int_{C} \prod_{i=1}^{2 N-1} \Gamma\left(\frac{\sqrt{p q}}{t_{i}} y^{ \pm 1}\right) \frac{d y}{2 \pi \mathrm{i} y}
$$

where is it assumed that $N \geq 2$. Here the balancing condition reads $\prod_{m=1}^{2 N-1} t_{m}=1$, so that at least one of the parameters should be of modulus greater than 1. Therefore the integration contours in $I_{E}$ should be deformed appropriately. For the gauge group $S O(2 N)$ we have SCI given by (2.4) with $N_{f}$ replaced by $2 N-2$ and

$$
I_{M}^{S O(2)}=\prod_{1 \leq m<s \leq 2 N-2} \Gamma\left(t_{m} t_{s}\right) \prod_{i=1}^{2 N-2} \Gamma\left(t_{i}^{2}\right) \frac{(p ; p)_{\infty}(q ; q)_{\infty}}{2} \int_{\mathbb{T}}^{2 N-2} \prod_{i=1}^{N} \Gamma\left(\frac{\sqrt{p q}}{t_{i}} y^{ \pm 1}\right) \frac{d y}{2 \pi \mathrm{i} y}
$$

where the balancing condition is $\prod_{m=1}^{2 N-2} t_{m}=1$ and $N>2$. For $N=2$ both expressions diverge and one has to apply appropriate regularization $t_{1} t_{2} \neq 1$ and residue calculus to obtain a meaningful limit $t_{1} t_{2} \rightarrow 1$. Interestingly, both magnetic SCIs are represented by the general well-poised EHIs without the very-well-poisedness condition [114] (which is thus not obligatory for applications in supersymmetric theories).

Consider dualities for $G=S O(n)$ and $N_{f}=n-3$ [66]. Their SCIs are obtained by a reduction of the elliptic beta integral for $S P(2 N)$ group of type I as described above. For $S O(2 N+1)$-group with $2 N-2$ quarks the index is given in (2.5) with $N_{f}$ replaced by $2 N-2$ 
and the balancing condition $\prod_{m=1}^{2 N-2} t_{m}=(p q)^{-1 / 2}$ requiring a change of the integration contour. Due to the confinement the dual index has a simple form

$$
I_{M}=\prod_{1 \leq m<s \leq 2 N-2} \Gamma\left(t_{m} t_{s}\right) \prod_{i=1}^{2 N-2} \Gamma\left(t_{i}^{2}, \frac{\sqrt{p q}}{t_{i}}\right) .
$$

For the $S O(2 N)$-group the electric index has the form (2.4) with $N_{f}$ replaced by $2 N-3$ and the balancing condition $\prod_{m=1}^{2 N-3} t_{m}=(p q)^{-1 / 2}$. Its magnetic partner is

$$
I_{M}=\prod_{1 \leq m<s \leq 2 N-3} \Gamma\left(t_{m} t_{s}\right) \prod_{i=1}^{2 N-3} \Gamma\left(t_{i}^{2}, \frac{\sqrt{p q}}{t_{i}}\right)
$$

Extra terms $\prod_{i=1}^{2 N-3} \Gamma\left(\frac{\sqrt{p q}}{t_{i}}\right)$ appear in (2.12) from the fundamental representation, although the dual gauge group is absent being formally defined as $S O(1)$.

Similarly one can consider the case of $G=S O(n)$ with $N_{f}=n-4$ [66]. For $S O(2 N+1)$-group SCI has the form (2.5) with $N_{f}$ replace by $2 N-3$ and the balancing condition $\prod_{m=1}^{2 N-3} t_{m}=$ $(p q)^{-1}$. In the infrared region particles confine and

$$
I_{M}=\prod_{1 \leq m<s \leq 2 N-3} \Gamma\left(t_{m} t_{s}\right) \prod_{i=1}^{2 N-3} \Gamma\left(t_{i}^{2}\right)
$$

For $S O(2 N)$-group electric SCI has the form (2.5) with $N_{f}$ replaced by $2 N-4$ and the balancing condition $\prod_{m=1}^{2 N-4} t_{m}=(p q)^{-1}$. Its dual has the form

$$
I_{M}=\prod_{1 \leq m<s \leq 2 N-4} \Gamma\left(t_{m} t_{s}\right) \prod_{i=1}^{2 N-4} \Gamma\left(t_{i}^{2}\right)
$$

2.2. Connection to the Witten anomaly. The even-dimensional theories have triangle anomalies associated with the global currents. For odd-dimensional field theories these anomalies are absent and this fact plays a negative role in searching corresponding dualities (because of the absence of powerful 't Hooft anomaly matching conditions). That is why the reduction of $4 d$ SCIs to $3 d$ partition functions discovered in [37] is important for searching $3 d$ dualities, since it inherits the information hidden in higher dimensional anomaly matching conditions.

However, apart from the global triangle anomalies there is a non-perturbative anomaly found by Witten [125], which is associated with the fact that the fourth homotopy group is non-trivial for some gauge groups. For examples, it was found that an $S U(2)$ gauge group theory with odd number of fermions is not well defined because $\pi^{4}(S U(2))=\mathbb{Z}_{2}$. The same argument applies to supersymmetric field theories. Therefore it is important to understand how this anomaly manifests itself in SCIs and we analyze this question below.

We start from an example of the $s$-confining theory: $4 d \mathcal{N}=1$ SYM theory with $S U(2)$ gauge group and 6 chiral superfields. The confining phase contains baryons $M_{i j}$ forming the antisymmetric tensor of the flavor group $S U(6)$. Corresponding SCIs were discussed in [36, 117] and they are given by the left- and right-hand sides of the elliptic beta integral [110]. So, the electric SCI has the form

$$
I_{E}\left(s_{1}, \ldots, s_{6}\right)=\frac{(p ; p)_{\infty}(q ; q)_{\infty}}{2} \int_{\mathbb{T}} \frac{\prod_{i=1}^{6} \Gamma\left(s_{i} z^{ \pm 1}\right)}{\Gamma\left(z^{ \pm 2}\right)} \frac{d z}{2 \pi \mathrm{i} z}
$$


with the balancing condition $\prod_{i=1}^{6} s_{i}=p q$. Changing the integration variable $z \rightarrow-z$ we see that $I_{E}\left(s_{1}, \ldots, s_{6}\right)=I_{E}\left(-s_{1}, \ldots,-s_{6}\right)$. The magnetic SCI is $I_{M}=\prod_{1 \leq j<k \leq 6} \Gamma\left(s_{j} s_{k}\right)=I_{E}$.

Let us set $s_{6}=\sqrt{p q}$. From the reflection equation for the elliptic gamma function one has $\Gamma\left(\sqrt{p q} z^{ \pm 1}\right)=1$. Therefore the reduced SCI takes the form

$$
I_{E 1}\left(s_{1}, \ldots, s_{5}\right)=\frac{(p ; p)_{\infty}(q ; q)_{\infty}}{2} \int_{\mathbb{T}} \frac{\prod_{i=1}^{5} \Gamma\left(s_{i} z^{ \pm 1}\right)}{\Gamma\left(z^{ \pm 2}\right)} \frac{d z}{2 \pi \mathrm{i} z},
$$

where the balancing condition is $\prod_{i=1}^{5} s_{i}=\sqrt{p q}$. According to the prescription for constructing SCIs, this expression describes $\mathcal{N}^{i=1}=1$ SYM theory with $S U(2)$ gauge group and 5 quarks forming a fundamental representation of the flavor group $S U(5)$ and having the $R$-charges $2 r=1 / 5$. The situation looks as if one of the quarks has been integrated out. As to the magnetic SCI, it takes the form

$$
I_{M 1}\left(s_{1}, \ldots, s_{5}\right)=\prod_{1 \leq i<j \leq 5} \Gamma\left(s_{i} s_{j}\right) \prod_{i=1}^{5} \Gamma\left(\sqrt{p q} s_{i}\right)
$$

and describes a confined theory of two types of mesons - the antisymmetric tensor representation $T_{A}$ of the group $S U(5)$ with the $R$-charge $2 / 5$ and the fundamental representation of $S U(5)$ with the $R$-charge $6 / 5$. As a consequence of the superconformal algebra, formal canonical dimension of the latter field is bigger than 1, i.e. formally the unitarity is broken, but real physical content of formally dual theories outside conformal windows require better understanding.

So, the electric theory has the Witten anomaly and the magnetic theory has problems with the unitarity. Despite of the non-physical properties, these theories are presumably dual to each other since all known duality tests are valid for them, including the equality of SCIs. A natural question is whether SCI feels in any way this anomaly ambiguity or not? As argued in [125], physical observables in this anomalous theory should vanish due to the cancellation induced by the "large" gauge transformations which change the sign of the fermion determinant. This means that SCI should vanish as well, as a gauge invariant object. However, SCI we use was computed basically from the free field theory (in a sense, perturbatively), and the nonperturbative effect of the large gauge transformation do not enter it, yielding a nonzero result.

Still, we believe that SCIs catch this effect. For instance, in the above confining theory with 5 quarks $I_{E 1}\left(s_{1}, \ldots, s_{5}\right) \neq I_{E 1}\left(-s_{1}, \ldots,-s_{5}\right)$, since the balancing condition is not preserved by the reflections $s_{j} \rightarrow-s_{j}$. There is an ambiguity in reducing the number of quarks: one can choose $s_{6}=-\sqrt{p q}$ and obtain SCI of the same shape (2.16), but with the balancing condition having the different sign $\prod_{i=1}^{5} s_{i}=-\sqrt{p q}$. We interpret this ambiguity in reductions together with the breaking of the reflection symmetry $s_{j} \rightarrow-s_{j}$ as manifestations of the Witten anomaly.

For instance, if we choose in the elliptic beta integral $s_{6}=\sqrt{p q}$ and $s_{5}=-\sqrt{p q}$, we obtain the relation

$$
\begin{aligned}
I_{E 2}= & \frac{(p ; p)_{\infty}(q ; q)_{\infty}}{2} \int_{\mathcal{C}} \frac{\prod_{k=1}^{4} \Gamma\left(s_{k} z^{ \pm 1} ; p, q\right)}{\Gamma\left(z^{ \pm 2} ; p, q\right)} \frac{d z}{2 \pi \mathrm{i} z} \\
& =I_{M 2}=2(-p ; p)_{\infty}(-q ; q)_{\infty} \prod_{1 \leq j<k \leq 4} \Gamma\left(s_{j} s_{k}\right) \prod_{k=1}^{4} \Gamma\left(p q s_{k}^{2} ; p^{2}, q^{2}\right),
\end{aligned}
$$

where $\prod_{k=1}^{4} s_{k}=-1$ and the contour $\mathcal{C}$ is chosen appropriately. (There is a misprint in the corresponding equality given before formula (4.9) in [114] - the infinite products independent on $s_{j}$ were combined there in an erroneous way.) If we interpret this relation as the equality of 
superconformal indices for some confining theory with four quarks, then the Witten anomaly is absent and, indeed, $I_{E 2}\left(s_{1}, \ldots, s_{4}\right)=I_{E 2}\left(-s_{1}, \ldots,-s_{4}\right)$. The physical meaning of this duality is not quite clear since the standard Römelsberger prescription does not apply to it. Namely, the electric theory has four quarks, but some nontrivial topological contributions to SCI are present leading to the non-standard balancing condition indicating on a non-marginal deformation of the standard four quarks electric theory.

The described effect exists only for $\mathcal{N}=1$ SYM theories with $S P(2 N)$ (and $S U(2)$ ) gauge group theories. The $G=S O(n)$ theories do not have such a problem since $\pi^{4}(S O(n))=1$. The flavor symmetry group in this case is $S U\left(N_{f}\right)$ (instead of $S U\left(2 N_{f}\right)$ ) and one can integrate out a single quark field without problems. At the level of SCIs this is reached by restricting one of the fugacities in an appropriate way.

2.3. $S O / S P$ gauge group theories with small number of flavors. Here we consider relations between $\mathcal{N}=1 \mathrm{SYM}$ theories with orthogonal and symplectic gauge groups with small number of flavors. Take the dualities for $S P(2)$ gauge group theory with 8 quarks. This model was suggested in [24] and studied in detail in [116], where it was argued that there are in total 72 dual theories having specific physical manifestations [74].

Electric theory SCI is described by an elliptic analogue of the Euler-Gauss hypergeometric function introduced in [111, 112]

$$
V\left(t_{1}, \ldots, t_{8} ; p, q\right)=\frac{(p ; p)_{\infty}(q ; q)_{\infty}}{2} \int_{\mathbb{T}} \frac{\prod_{j=1}^{8} \Gamma\left(t_{j} z^{ \pm 1}\right)}{\Gamma\left(z^{ \pm 2}\right)} \frac{d z}{2 \pi \mathrm{i} z}
$$

with the constraints $\left|t_{j}\right|<1$ for eight complex variables $t_{1}, \ldots, t_{8}$ and the balancing condition $\prod_{j=1}^{8} t_{j}=(p q)^{2}$. This function obeys the following symmetry transformation derived in [11]

$$
V\left(t_{1}, \ldots, t_{8} ; p, q\right)=\prod_{1 \leq j<k \leq 4} \Gamma\left(t_{j} t_{k}, t_{j+4} t_{k+4}\right) V\left(s_{1}, \ldots, s_{8} ; p, q\right),
$$

where complex variables $s_{j},\left|s_{j}\right|<1$, are connected with $t_{j}$ as follows

$$
\begin{aligned}
s_{j} & =\rho^{-1} t_{j}, j=1,2,3,4, \quad s_{j}=\rho t_{j}, j=5,6,7,8, \\
\rho & =\sqrt{\frac{t_{1} t_{2} t_{3} t_{4}}{p q}}=\sqrt{\frac{p q}{t_{5} t_{6} t_{7} t_{8}}} .
\end{aligned}
$$

This fundamental relation taken together with the evident $S_{8}$-permutational group of symmetries in parameters $t_{j}$ generates the Weyl group $W\left(E_{7}\right)$ [97].

Let us apply the following constraint on the parameters

$$
t_{3,4,5,6,7,8}=\{ \pm \sqrt{p}, \pm \sqrt{q},-1,-\sqrt{p q}\} .
$$

The initial electric SCI takes the form

$$
I_{E}=\frac{(p ; p)_{\infty}(q ; q)_{\infty}}{2} \int_{\mathbb{T}} \frac{\prod_{i=1}^{2} \Gamma\left(t_{i} z^{ \pm 1}\right)}{\Gamma\left(z^{ \pm 1}\right)} \frac{d z}{2 \pi \mathrm{i} z}
$$

where $t_{1} t_{2}=\sqrt{p q}$, while in the magnetic SCIs $S_{8}$-symmetry is explicitly broken and we can get various inequivalently looking expressions. E.g., split the initial 8 parameters into two sets

$$
\left\{ \pm \sqrt{q},-\sqrt{p q}, t_{1}\right\} \text { and }\left\{ \pm \sqrt{p},-1, t_{2}\right\}
$$

for which $\rho=\sqrt{t_{1}}(q / p)^{1 / 4}$. In terms of the parameters

$$
s_{1,2,3,4}=\rho^{-1}\left\{ \pm \sqrt{q},-\sqrt{p q}, t_{1}\right\} \text { and } s_{5,6,7,8}=\rho\left\{ \pm \sqrt{p},-1, t_{2}\right\}
$$


the magnetic SCI takes a quite simple form

$$
I_{M}=\frac{(p ; p)_{\infty}(q ; q)_{\infty}}{2} \int_{\mathbb{T}} \frac{\prod_{i=1}^{8} \Gamma\left(s_{i} z^{ \pm 1}\right)}{\Gamma\left(z^{ \pm 2}\right)} \frac{d z}{2 \pi \mathrm{i} z} .
$$

After multiplication of both $I_{E}$ and $I_{M}$ by $\prod_{i=1,2} \Gamma\left(t_{i}\right)$, on the electric side we obtain SCI for $\mathcal{N}=1 \mathrm{SYM}$ with $S O(3)$ gauge group with two quarks and on the magnetic side we have SCI of a $\mathcal{N}=1$ SYM theory with $S P(2)$ gauge group and eight quarks whose flavor fugacities are chosen in a special way. This relation can be generalized to arbitrary number of colors $N$ and to the theories discussed in Sect. 2.1. However, the general meaning of all such relations is not clear yet.

\section{S-CONFINING THEORIES WITH THE SPINOR MATTER}

In this chapter we systematically consider all known $s$-confining theories with $S O(N)$-gauge groups and the matter in spinor representation [23]. The upper parts of the tables contain information on the charges and field representation types of the electric models (except of the vector superfield). The lower parts of the tables describe the $s$-confining phase of the theory. The models with the rank of the gauge group smaller than 4 are not considered because of different isomorphisms for orthogonal groups: $S O(6) \simeq S U(4), S O(5) \simeq S P(4), S O(4) \simeq$ $S U(2) \times S U(2), S O(3) \simeq S U(2)$, and $S O(2) \simeq U(1)$.

For the orthogonal group $S O(2 N)$ there are two types of spinor representations: the proper spinor representation, which we denote as $s$, and its complex conjugate which is denoted as $c$, both representations have dimension $2^{N-1}$. For gauge group $S O(2 N+1)$ there exists only the spinor representation $s$ which has the dimension $2^{N}$. Characters of the corresponding representations can be found in the Appendix.

\subsection{Confinement for $S O(7)$ gauge group.}

3.1.1. $S U(6)$ flavor symmetry group. The matter field content is [23]

\begin{tabular}{|c|c|c|c|}
\hline & $S O(7)$ & $S U(6)$ & $U(1)_{R}$ \\
\hline$S$ & $s$ & $f$ & $2 r=\frac{1}{6}$ \\
\hline \hline$S^{2}$ & & $T_{S}$ & $\frac{1}{3}$ \\
$S^{4}$ & & $\bar{T}_{A}$ & $\frac{2}{3}$ \\
\hline
\end{tabular}

Corresponding SCIs have the form

$$
I_{E}=\frac{(p ; p)_{\infty}^{3}(q ; q)_{\infty}^{3}}{2^{3} 3 !} \int_{\mathbb{T}^{3}} \frac{\prod_{i=1}^{6} \Gamma\left(s_{i}\left(z_{1} z_{2} z_{3}\right)^{ \pm 1}\right) \prod_{j=1}^{3} \Gamma\left(s_{i}\left(z_{j}^{-2} z_{1} z_{2} z_{3}\right)^{ \pm 1}\right)}{\prod_{j=1}^{3} \Gamma\left(z_{j}^{ \pm 2}\right) \prod_{1 \leq j<k \leq 3} \Gamma\left(z_{j}^{ \pm 2} z_{k}^{ \pm 2}\right)} \prod_{j=1}^{3} \frac{d z_{j}}{2 \pi \mathrm{i} z_{j}},
$$

where $\left|s_{i}\right|<1$ with the balancing condition $\prod_{i=1}^{6} s_{i}=(p q)^{1 / 2}$, and

$$
I_{M}=\prod_{i=1}^{6} \Gamma\left(s_{i}^{2}\right) \prod_{1 \leq i<j \leq 6} \Gamma\left(s_{i} s_{j},(p q)^{\frac{1}{2}} s_{i}^{-1} s_{j}^{-1}\right) .
$$

In the limit $p=q=0$ (after proper treatment of the balancing condition) and $s_{2,3,4,5}=0$ the equality $I_{E}=I_{M}$ is directly verified by residue calculus.

This and all other dualities described in this paper satisfy the 't Hooft anomaly matching conditions. According to [120] this means that dual SCIs have the same $S L(3, \mathbb{Z})$-modular group properties (in particular, one can associate with these dualities some totally elliptic hypergeometric terms). 
3.1.2. $S U(5) \times U(1)$ flavor group. The matter content is [23]

\begin{tabular}{|c|c|c|c|c|}
\hline & $S O(7)$ & $S U(5)$ & $U(1)$ & $U(1)_{R}$ \\
\hline$S$ & $s$ & $f$ & 1 & 0 \\
$Q$ & $f$ & 1 & -5 & 1 \\
\hline \hline$Q^{2}$ & & 1 & -10 & 2 \\
$S^{2}$ & & $T_{S}$ & 2 & 0 \\
$S^{4}$ & & $\bar{f}$ & 4 & 0 \\
$S^{2} Q$ & & $T_{A}$ & -3 & 1 \\
$S^{4} Q$ & & $\bar{f}$ & -1 & 1 \\
\hline
\end{tabular}

Corresponding SCIs are

$$
\begin{gathered}
I_{E}=\frac{(p ; p)_{\infty}^{3}(q ; q)_{\infty}^{3}}{2^{3} 3 !} \Gamma(t) \int_{\mathbb{T}^{3}} \frac{\prod_{j=1}^{3} \Gamma\left(t z_{j}^{ \pm 2}\right)}{\prod_{j=1}^{3} \Gamma\left(z_{j}^{ \pm 2}\right) \prod_{1 \leq j<k \leq 3} \Gamma\left(z_{j}^{ \pm 2} z_{k}^{ \pm 2}\right)} \\
\times \prod_{i=1}^{5} \Gamma\left(s_{i}\left(z_{1} z_{2} z_{3}\right)^{ \pm 1}\right) \prod_{j=1}^{3} \Gamma\left(s_{i}\left(\frac{z_{j}^{2}}{z_{1} z_{2} z_{3}}\right)^{ \pm 1}\right) \prod_{j=1}^{3} \frac{d z_{j}}{2 \pi \mathrm{i} z_{j}},
\end{gathered}
$$

where $\left|s_{i}\right|<1$ with the balancing condition $t \prod_{i=1}^{5} s_{i}=\sqrt{p q}$, and

$$
I_{M}=\Gamma\left(t^{2}\right) \prod_{i=1}^{5} \Gamma\left(\frac{\sqrt{p q}}{s_{i} t}, \frac{\sqrt{p q}}{s_{i}}, s_{i}^{2}\right) \prod_{1 \leq i<j \leq 5} \Gamma\left(s_{i} s_{j}, t s_{i} s_{j}\right) .
$$

Again, this $s$-confining duality predicts the exact integration formula $I_{E}=I_{M}$. Similar to the previous case, this identity is easily checked in the limit $p=q=0$ and $s_{2,3,4}=0$.

3.1.3. $S U(4) \times S U(2) \times U(1)$ flavor group. The matter content is 23 .

\begin{tabular}{|c|c|c|c|c|c|}
\hline & $S O(7)$ & $S U(4)$ & $S U(2)$ & $U(1)$ & $U(1)_{R}$ \\
\hline$S$ & $s$ & $f$ & 1 & 1 & 0 \\
$Q$ & $f$ & 1 & $f$ & -2 & $\frac{1}{2}$ \\
\hline \hline$Q^{2}$ & & 1 & $T_{S}$ & -4 & 1 \\
$S^{2}$ & & $T_{S}$ & 1 & 2 & 0 \\
$S^{2} Q$ & & $T_{A}$ & $f$ & 0 & $\frac{1}{2}$ \\
$S^{2} Q^{2}$ & & $T_{A}$ & 1 & -2 & 1 \\
$S^{4}$ & & 1 & 1 & 4 & 0 \\
$S^{4} Q$ & & 1 & $f$ & 2 & $\frac{1}{2}$ \\
\hline
\end{tabular}

Corresponding SCIs are

$$
\begin{gathered}
I_{E}=\frac{(p ; p)_{\infty}^{3}(q ; q)_{\infty}^{3}}{2^{3} 3 !} \prod_{i=1}^{2} \Gamma\left(t_{i}\right) \int_{\mathbb{T}^{3}} \frac{\prod_{i=1}^{2} \prod_{j=1}^{3} \Gamma\left(t_{i} z_{j}^{ \pm 2}\right)}{\prod_{j=1}^{3} \Gamma\left(z_{j}^{ \pm 2}\right) \prod_{1 \leq j<k \leq 3} \Gamma\left(z_{j}^{ \pm 2} z_{k}^{ \pm 2}\right)} \\
\times \prod_{i=1}^{4} \Gamma\left(s_{i}\left(z_{1} z_{2} z_{3}\right)^{ \pm 1}\right) \prod_{j=1}^{3} \Gamma\left(s_{i}\left(\frac{z_{j}^{2}}{z_{1} z_{2} z_{3}}\right)^{ \pm 1}\right) \prod_{j=1}^{3} \frac{d z_{j}}{2 \pi \mathrm{i} z_{j}},
\end{gathered}
$$

where $\left|s_{i}\right|,\left|t_{j}\right|<1, s t=\sqrt{p q}$ with $s=\prod_{i=1}^{4} s_{i}, t=\prod_{i=1}^{2} t_{i}$, and

$$
I_{M}=\Gamma(s, t) \prod_{i=1}^{2} \Gamma\left(s t_{i}, t_{i}^{2}\right) \prod_{i=1}^{4} \Gamma\left(s_{i}^{2}\right) \prod_{1 \leq i<j \leq 4} \Gamma\left(s_{i} s_{j}, t s_{i} s_{j}\right) \prod_{k=1}^{2} \Gamma\left(s_{i} s_{j} t_{k}\right) .
$$


3.1.4. $S U(3) \times S U(3) \times U(1)$ flavor group. The matter content is [23]

\begin{tabular}{|c|c|c|c|c|c|}
\hline & $S O(7)$ & $S U(3)$ & $S U(3)$ & $U(1)$ & $U(1)_{R}$ \\
\hline$S$ & $s$ & $f$ & 1 & 1 & 0 \\
$Q$ & $f$ & 1 & $f$ & -1 & $\frac{1}{3}$ \\
\hline \hline$Q^{2}$ & & 1 & $T_{S}$ & -2 & $\frac{2}{3}$ \\
$S^{2}$ & & $T_{S}$ & 1 & 2 & 0 \\
$S^{2} Q$ & & $f=T_{A}$ & $f$ & 1 & $\frac{1}{3}$ \\
$S^{2} Q^{2}$ & & $\bar{f}$ & $\bar{f}$ & 0 & $\frac{2}{3}$ \\
$S^{2} Q^{3}$ & & $T_{S}$ & 1 & -1 & 1 \\
\hline
\end{tabular}

Corresponding SCIs are

$$
\begin{gathered}
I_{E}=\frac{(p ; p)_{\infty}^{3}(q ; q)_{\infty}^{3}}{2^{3} 3 !} \prod_{i=1}^{3} \Gamma\left(t_{i}\right) \int_{\mathbb{T}^{3}} \frac{\prod_{i=1}^{3} \prod_{j=1}^{3} \Gamma\left(t_{i} z_{j}^{ \pm 2}\right)}{\prod_{j=1}^{3} \Gamma\left(z_{j}^{ \pm 2}\right) \prod_{1 \leq j<k \leq 3} \Gamma\left(z_{j}^{ \pm 2} z_{k}^{ \pm 2}\right)} \\
\times \prod_{i=1}^{3} \Gamma\left(s_{i}\left(z_{1} z_{2} z_{3}\right)^{ \pm 1}\right) \prod_{i, j=1}^{3} \Gamma\left(s_{i}\left(\frac{z_{j}^{2}}{z_{1} z_{2} z_{3}}\right)^{ \pm 1}\right) \prod_{j=1}^{3} \frac{d z_{j}}{2 \pi \mathrm{i} z_{j}}
\end{gathered}
$$

where $\left|s_{i}\right|,\left|t_{j}\right|<1, s t=\sqrt{p q}$ with $s=\prod_{i=1}^{3} s_{i}, t=\prod_{i=1}^{3} t_{i}$, and

$$
I_{M}=\prod_{i=1}^{3} \Gamma\left(s_{i}^{2}, t_{i}^{2}, t s_{i}^{2}\right) \prod_{i, j=1}^{3} \Gamma\left(s t s_{i}^{-1} t_{j}^{-1}, s s_{i}^{-1} t_{j}\right) \prod_{1 \leq i<j \leq 3} \Gamma\left(s_{i} s_{j}, t_{i} t_{j}, t s_{i} s_{j}\right) .
$$

3.2. $G=S O(8)$.

3.2.1. $S U(4) \times S U(3) \times U(1)$ flavor group. The matter content is [23]

\begin{tabular}{|c|c|c|c|c|c|}
\hline & $S O(8)$ & $S U(4)$ & $S U(3)$ & $U(1)$ & $U(1)_{R}$ \\
\hline$Q$ & $f$ & $f$ & 1 & 3 & $\frac{1}{4}$ \\
$S$ & $s$ & 1 & $f$ & -4 & 0 \\
\hline \hline$Q^{2}$ & & $T_{S}$ & 1 & 6 & $\frac{1}{2}$ \\
$S^{2}$ & & 1 & $T_{S}$ & -8 & 0 \\
$S^{2} Q^{2}$ & & $T_{A}$ & $\bar{f}$ & -2 & $\frac{1}{2}$ \\
$S^{2} Q^{4}$ & & 1 & $T_{S}$ & 4 & 1 \\
\hline
\end{tabular}

Corresponding SCIs are

$$
\begin{gathered}
I_{E}=\frac{(p ; p)_{\infty}^{4}(q ; q)_{\infty}^{4}}{2^{3} 4 !} \int_{\mathbb{T}^{4}} \frac{\prod_{i=1}^{4} \prod_{j=1}^{4} \Gamma\left(s_{i} z_{j}^{ \pm 2}\right)}{\prod_{1 \leq j<k \leq 4} \Gamma\left(z_{j}^{ \pm 2} z_{k}^{ \pm 2}\right)} \\
\times \prod_{i=1}^{3} \Gamma\left(t_{i}\left(z_{1} z_{2} z_{3} z_{4}\right)^{ \pm 1}\right) \prod_{i=1}^{3} \prod_{1 \leq j<k \leq 4} \Gamma\left(t_{i} \frac{z_{j}^{2} z_{k}^{2}}{z_{1} z_{2} z_{3} z_{4}}\right) \prod_{j=1}^{4} \frac{d z_{j}}{2 \pi \mathrm{i} z_{j}}
\end{gathered}
$$

where $\left|s_{i}\right|,\left|t_{j}\right|<1$, and

$$
I_{M}=\prod_{i=1}^{3} \Gamma\left(t_{i}^{2}, s t_{i}^{2}\right) \prod_{i=1}^{4} \Gamma\left(s_{i}^{2}\right) \prod_{1 \leq i<j \leq 3} \Gamma\left(t_{i} t_{j}, s t_{i} t_{j}\right) \prod_{1 \leq i<j \leq 4}\left(\Gamma\left(s_{i} s_{j}\right) \prod_{k=1}^{3} \Gamma\left(t s_{i} s_{j} t_{k}^{-1}\right)\right),
$$

with $s=\prod_{i=1}^{4} s_{i}, t=\prod_{i=1}^{3} t_{i}$, and the balancing condition $s t=\sqrt{p q}$. A simple check of the equality of these SCIs is obtained in the limit $p=q=0$ and $s_{2,3,4}=t_{2}=0$. 
3.2.2. $S U(4) \times S U(2) \times U(1)_{1} \times U(1)_{2}$ flavor group. The matter content is [23]

\begin{tabular}{|c|c|c|c|c|c|c|}
\hline & $S O(8)$ & $S U(4)$ & $S U(2)$ & $U(1)_{1}$ & $U(1)_{2}$ & $U(1)_{R}$ \\
\hline$Q$ & $f$ & $f$ & 1 & 1 & 0 & $\frac{1}{4}$ \\
$S$ & $s$ & 1 & $f$ & -2 & 1 & 0 \\
$S^{\prime}$ & $c$ & 1 & 1 & 0 & -2 & 0 \\
\hline \hline$Q^{2}$ & & $T_{S}$ & 1 & 2 & 0 & $\frac{1}{2}$ \\
$S^{2}$ & & 1 & $T_{S}$ & -4 & 2 & 0 \\
$S^{\prime 2}$ & & 1 & 1 & 0 & -4 & 0 \\
$S^{2} Q^{2}$ & & $T_{A}$ & 1 & -2 & 2 & $\frac{1}{2}$ \\
$S^{2} Q^{4}$ & & 1 & $T_{S}$ & 0 & 2 & 1 \\
$S^{\prime 2} Q^{4}$ & & 1 & 1 & 4 & -4 & 1 \\
$S S^{\prime} Q$ & & $f$ & $f$ & -1 & -1 & $\frac{1}{4}$ \\
$S S^{\prime} Q^{3}$ & & $\frac{f}{f}$ & $f$ & 1 & -1 & $\frac{3}{4}$ \\
\hline
\end{tabular}

Corresponding SCIs are

$$
\begin{aligned}
I_{E} & =\frac{(p ; p)_{\infty}^{4}(q ; q)_{\infty}^{4}}{2^{3} 4 !} \int_{\mathbb{T}^{4}} \frac{\prod_{i, j=1}^{4} \Gamma\left(s_{i} z_{j}^{ \pm 2}\right)}{\prod_{1 \leq j<k \leq 4} \Gamma\left(z_{j}^{ \pm 2} z_{k}^{ \pm 2}\right)} \prod_{i=1}^{2} \Gamma\left(t_{i}\left(z_{1} z_{2} z_{3} z_{4}\right)^{ \pm 1}\right) \\
& \times \prod_{i=1}^{2} \prod_{1 \leq j<k \leq 4} \Gamma\left(t_{i} \frac{z_{j}^{2} z_{k}^{2}}{z_{1} z_{2} z_{3} z_{4}}\right) \prod_{j=1}^{4} \Gamma\left(u\left(\frac{z_{j}^{2}}{z_{1} z_{2} z_{3} z_{4}}\right)^{ \pm 1}\right) \prod_{j=1}^{4} \frac{d z_{j}}{2 \pi \mathrm{i} z_{j}}
\end{aligned}
$$

where $\left|s_{i}\right|,\left|t_{j}\right|,|u|<1$, stu $=\sqrt{p q}$ with $s=\prod_{i=1}^{4} s_{i}, t=\prod_{i=1}^{2} t_{i}$, and

$$
I_{M}=\Gamma\left(u^{2}, s u^{2}, t, s t\right) \prod_{j=1}^{2}\left(\Gamma\left(t_{j}^{2}, s t_{j}^{2}\right) \prod_{i=1}^{4} \Gamma\left(u s_{i} t_{j}, \frac{u s}{s_{i}} t_{j}\right)\right) \prod_{i=1}^{4} \Gamma\left(s_{i}^{2}\right) \prod_{1 \leq i<j \leq 4} \Gamma\left(s_{i} s_{j}, t s_{i} s_{j}\right) .
$$

3.2.3. $S U(3) \times S U(3) \times U(1)_{1} \times U(1)_{2}$ flavor group. The matter content is [23]

\begin{tabular}{|c|c|c|c|c|c|c|}
\hline & $S O(8)$ & $S U(3)$ & $S U(3)$ & $U(1)_{1}$ & $U(1)_{2}$ & $U(1)_{R}$ \\
\hline$Q$ & $f$ & 1 & 1 & 0 & 6 & 1 \\
$S$ & $s$ & $f$ & 1 & 1 & -1 & 0 \\
$S^{\prime}$ & $c$ & 1 & $f$ & -1 & -1 & 0 \\
\hline \hline$Q^{2}$ & & 1 & 1 & 0 & 12 & 2 \\
$S^{2}$ & & $T_{S}$ & 1 & 2 & -2 & 0 \\
$S^{\prime 2}$ & & 1 & $T_{S}$ & -2 & -2 & 0 \\
$S S^{\prime} Q$ & & $f$ & $f$ & 0 & 4 & 1 \\
$S^{3} S^{\prime} Q$ & & 1 & $f$ & 2 & 2 & 1 \\
$S S^{\prime 3} Q$ & & $f$ & 1 & -2 & 2 & 1 \\
$S^{2} S^{\prime 2}$ & & $\frac{f}{f}$ & $\frac{f}{4}$ & -4 & 0 \\
\hline
\end{tabular}

Corresponding SCIs are

$$
\begin{aligned}
& I_{E}=\frac{(p ; p)_{\infty}^{4}(q ; q)_{\infty}^{4}}{2^{3} 4 !} \int_{\mathbb{T}^{4}} \frac{\prod_{j=1}^{4} \Gamma\left(u z_{j}^{ \pm 2}\right)}{\prod_{1 \leq j<k \leq 4} \Gamma\left(z_{j}^{ \pm 2} z_{k}^{ \pm 2}\right)} \prod_{i=1}^{3} \Gamma\left(s_{i}\left(z_{1} z_{2} z_{3} z_{4}\right)^{ \pm 1}\right) \\
& \quad \times \prod_{i=1}^{3} \prod_{1 \leq j<k \leq 4} \Gamma\left(s_{i} \frac{z_{j}^{2} z_{k}^{2}}{z_{1} z_{2} z_{3} z_{4}}\right) \prod_{i=1}^{3} \prod_{j=1}^{4} \Gamma\left(t_{i}\left(\frac{z_{j}^{2}}{z_{1} z_{2} z_{3} z_{4}}\right)^{ \pm 1}\right) \prod_{j=1}^{4} \frac{d z_{j}}{2 \pi \mathrm{i} z_{j}},
\end{aligned}
$$


where $\left|s_{i}\right|,\left|t_{j}\right|,|u|<1$, and

$$
I_{M}=\Gamma\left(u^{2}\right) \prod_{i=1}^{3} \Gamma\left(s_{i}^{2}, t_{i}^{2}, s u t_{i}, t u s_{i}\right) \prod_{i, j=1}^{3} \Gamma\left(u s_{i} t_{j}, s t s_{i}^{-1} t_{j}^{-1}\right) \prod_{1 \leq i<j \leq 3} \Gamma\left(s_{i} s_{j}, t_{i} t_{j}\right),
$$

with $s=\prod_{i=1}^{3} s_{i}, t=\prod_{i=1}^{3} t_{i}$, and the balancing condition $s t u=\sqrt{p q}$. We checked the equality of these SCIs in the limit $p=q=0$ and $s_{2,3}=t_{2} \rightarrow 0$.

3.2.4. $S U(3) \times S U(2)_{1} \times S U(2)_{2} \times U(1)_{1} \times U(1)_{2}$ flavor group. The matter content is [23]

\begin{tabular}{|c|c|c|c|c|c|c|c|}
\hline & $S O(8)$ & $S U(3)$ & $S U(2)_{1}$ & $S U(2)_{2}$ & $U(1)_{1}$ & $U(1)_{2}$ & $U(1)_{R}$ \\
\hline$Q$ & $f$ & $f$ & 1 & 1 & 0 & 4 & 0 \\
$S$ & $s$ & 1 & $f$ & 1 & 1 & -3 & $\frac{1}{4}$ \\
$S^{\prime}$ & $c$ & 1 & 1 & $f$ & -1 & -3 & $\frac{1}{4}$ \\
\hline \hline$Q^{2}$ & & $T_{S}$ & 1 & 1 & 0 & 8 & 0 \\
$S^{2}$ & & 1 & $T_{S}$ & 1 & 2 & -6 & $\frac{1}{2}$ \\
$S^{\prime 2}$ & & 1 & 1 & $T_{S}$ & -2 & -6 & $\frac{1}{2}$ \\
$S S^{\prime} Q$ & & $f$ & $f$ & $f$ & 0 & -2 & $\frac{1}{2}$ \\
$S^{2} Q^{2}$ & & $\frac{f}{f}$ & 1 & 1 & 2 & 2 & $\frac{1}{2}$ \\
$S^{\prime 2} Q^{2}$ & & $\frac{f}{2}$ & 1 & 1 & -2 & 2 & $\frac{1}{2}$ \\
$S S^{\prime} Q^{3}$ & & 1 & $f$ & $f$ & 0 & 6 & $\frac{1}{2}$ \\
$S^{2} S^{\prime 2}$ & & 1 & 1 & 1 & 0 & -12 & 1 \\
$S^{2} S^{\prime 2} Q^{2}$ & & $\frac{1}{f}$ & 1 & 1 & 0 & -4 & 1 \\
\hline
\end{tabular}

Corresponding SCIs are

$$
\begin{aligned}
& I_{E}=\frac{(p ; p)_{\infty}^{4}(q ; q)_{\infty}^{4}}{2^{3} 4 !} \int_{\mathbb{T}^{4}} \frac{\prod_{i=1}^{3} \prod_{j=1}^{4} \Gamma\left(s_{i} z_{j}^{ \pm 2}\right)}{\prod_{1 \leq j<k \leq 4} \Gamma\left(z_{j}^{ \pm 2} z_{k}^{ \pm 2}\right)} \prod_{i=1}^{2} \Gamma\left(t_{i}\left(z_{1} z_{2} z_{3} z_{4}\right)^{ \pm 1}\right) \\
& \quad \times \prod_{i=1}^{2} \prod_{1 \leq j<k \leq 4} \Gamma\left(t_{i} \frac{z_{j}^{2} z_{k}^{2}}{z_{1} z_{2} z_{3} z_{4}}\right) \prod_{i=1}^{2} \prod_{j=1}^{4} \Gamma\left(u_{i}\left(\frac{z_{j}^{2}}{z_{1} z_{2} z_{3} z_{4}}\right)^{ \pm 1}\right) \prod_{j=1}^{4} \frac{d z_{j}}{2 \pi \mathrm{i} z_{j}},
\end{aligned}
$$

where $\left|s_{i}\right|,\left|t_{i}\right|,\left|u_{i}\right|<1, s t u=\sqrt{p q}$ with $s=\prod_{i=1}^{3} s_{i}, t=\prod_{i=1}^{2} t_{i}, u=\prod_{i=1}^{2} u_{i}$, and

$$
\begin{gathered}
I_{M}=\Gamma(t, u, t u) \prod_{i=1}^{3} \Gamma\left(s_{i}^{2}\right) \prod_{i=1}^{2} \Gamma\left(t_{i}^{2}, u_{i}^{2}\right) \prod_{i, j=1}^{2} \Gamma\left(s t_{i} u_{j}\right) \\
\times \prod_{1 \leq i<j \leq 3} \Gamma\left(s_{i} s_{j}\right) \prod_{i=1}^{3} \Gamma\left(s t u s_{i}^{-1}, s t s_{i}^{-1}, s u s_{i}^{-1}\right) \prod_{i=1}^{3} \prod_{j, k=1}^{2} \Gamma\left(s_{i} t_{j} u_{k}\right) .
\end{gathered}
$$

3.3. $G=S O(9)$.

3.3.1. $S U(4)$ flavor group. The matter content is [23]

\begin{tabular}{|c|c|c|c|}
\hline & $S O(9)$ & $S U(4)$ & $U(1)_{R}$ \\
\hline$S$ & $s$ & $f$ & $\frac{1}{8}$ \\
\hline \hline$S^{2}$ & & $T_{S}$ & $\frac{1}{4}$ \\
$S^{4}$ & & $T_{A A S S}$ & $\frac{1}{2}$ \\
$S^{6}$ & & $T_{S}$ & $\frac{3}{4}$ \\
\hline
\end{tabular}


where $T_{A A S S}$ denotes the fourth rank tensor representation symmetric in two indices and antisymmetric in other two indices, whose character is given by the formula

$$
\chi_{T_{A A S S}, S U(4)}(\underline{s})=\sum_{1 \leq i<j \leq 4} s_{i}^{2} s_{j}^{2}+\sum_{i=1}^{4} \sum_{1 \leq j<k \leq 4 ; j, k \neq i} s_{i}^{2} s_{j} s_{k}+2 .
$$

Corresponding SCIs are

$$
\begin{gathered}
I_{E}=\frac{(p ; p)_{\infty}^{4}(q ; q)_{\infty}^{4}}{2^{4} 4 !} \int_{\mathbb{T}^{4}} \frac{\prod_{i=1}^{4} \Gamma\left(s_{i} z^{ \pm 1}\right) \prod_{i, j=1}^{4} \Gamma\left(s_{i}\left(\frac{z_{j}^{2}}{z}\right)^{ \pm 1}\right)}{\prod_{i=1}^{4} \Gamma\left(z_{i}^{ \pm 2}\right) \prod_{1 \leq j<k \leq 4} \Gamma\left(z_{j}^{ \pm 2} z_{k}^{ \pm 2}\right)} \\
\times \prod_{i=1}^{4} \prod_{1 \leq j<k \leq 4} \Gamma\left(s_{i} \frac{z_{j}^{2} z_{k}^{2}}{z}\right) \prod_{j=1}^{4} \frac{d z_{j}}{2 \pi \mathrm{i} z_{j}}
\end{gathered}
$$

where $z=z_{1} z_{2} z_{3} z_{4},\left|s_{i}\right|<1$, the balancing condition $s^{2}=\sqrt{p q}$ with $s=\prod_{i=1}^{4} s_{i}$, and

$$
I_{M}=\Gamma^{2}(s) \prod_{i=1}^{4} \Gamma\left(s_{i}^{2}, s s_{i}^{2}\right) \prod_{1 \leq i<j \leq 4} \Gamma\left(s_{i} s_{j}, s s_{i} s_{j}, s_{i}^{2} s_{j}^{2}\right) \prod_{i=1}^{4} \prod_{1 \leq j<k \leq 4 ; j, k \neq i} \Gamma\left(s_{i}^{2} s_{j} s_{k}\right) .
$$

3.3.2. $S U(3) \times S U(2) \times U(1)$ flavor group. The matter content is [23]

\begin{tabular}{|c|c|c|c|c|c|}
\hline & $S O(9)$ & $S U(3)$ & $S U(2)$ & $U(1)$ & $U(1)_{R}$ \\
\hline$S$ & $s$ & $f$ & 1 & 1 & 0 \\
$Q$ & $f$ & 1 & $f$ & -3 & $\frac{1}{2}$ \\
\hline \hline$Q^{2}$ & & 1 & $T_{S}$ & -6 & 1 \\
$S^{2} Q$ & & $T_{S}$ & $f$ & -1 & $\frac{1}{2}$ \\
$S^{2}$ & & $T_{S}$ & 1 & 2 & 0 \\
$S^{4}$ & & $\bar{T}_{S}$ & 1 & 4 & 0 \\
$S^{2} Q^{2}$ & & $\bar{f}$ & 1 & -4 & 1 \\
$S^{4} Q^{2}$ & & $f$ & 1 & -2 & 1 \\
$S^{4} Q$ & & $f$ & $f$ & 1 & $\frac{1}{2}$ \\
\hline
\end{tabular}

Corresponding SCIs are

$$
\begin{aligned}
I_{E}= & \frac{(p ; p)_{\infty}^{4}(q ; q)_{\infty}^{4}}{2^{4} 4 !} \prod_{i=1}^{2} \Gamma\left(t_{i}\right) \int_{\mathbb{T}^{4}} \frac{\prod_{i=1}^{2} \prod_{j=1}^{4} \Gamma\left(t_{i} z_{j}^{ \pm 2}\right) \prod_{i=1}^{3} \Gamma\left(s_{i} z^{ \pm 1}\right)}{\prod_{j=1}^{4} \Gamma\left(z_{j}^{ \pm 2}\right) \prod_{1 \leq j<k \leq 4} \Gamma\left(z_{j}^{ \pm 2} z_{k}^{ \pm 2}\right)} \\
& \times \prod_{i=1}^{3} \prod_{j=1}^{4} \Gamma\left(s_{i}\left(z_{j}^{2} z^{-1}\right)^{ \pm 1}\right) \prod_{i=1}^{3} \prod_{1 \leq j<k \leq 4} \Gamma\left(s_{i} z_{j}^{2} z_{k}^{2} z^{-1}\right) \prod_{j=1}^{4} \frac{d z_{j}}{2 \pi \mathrm{i} z_{j}},
\end{aligned}
$$

where $z=z_{1} z_{2} z_{3} z_{4},\left|s_{i}\right|<1, s^{2} t=\sqrt{p q}$ with $s=\prod_{i=1}^{3} s_{i}, t=\prod_{i=1}^{2} t_{i}$, and

$$
\begin{aligned}
I_{M}= & \prod_{i=1}^{2} \Gamma\left(t_{i}^{2}\right) \prod_{i=1}^{3} \Gamma\left(s_{i}^{2}, s t s_{i}, s^{2} s_{i}^{-1}, s t s_{i}^{-1}\right) \prod_{i=1}^{3} \prod_{j=1}^{2} \Gamma\left(s_{i}^{2} t_{j}, s s_{i} t_{j}\right) \\
& \times \Gamma(t) \prod_{1 \leq i<j \leq 3} \Gamma\left(s_{i} s_{j}, s^{2} s_{i}^{-1} s_{j}^{-1}\right) \prod_{1 \leq i<j \leq 3} \prod_{k=1}^{2} \Gamma\left(s_{i} s_{j} t_{k}\right) .
\end{aligned}
$$


3.3.3. $S U(2) \times S U(4) \times U(1)$ flavor group. The matter content is [23]

\begin{tabular}{|c|c|c|c|c|c|}
\hline & $S O(9)$ & $S U(2)$ & $S U(4)$ & $U(1)$ & $U(1)_{R}$ \\
\hline$S$ & $s$ & $f$ & 1 & 1 & $\frac{1}{4}$ \\
$Q$ & $f$ & 1 & $f$ & -1 & 0 \\
\hline \hline$Q^{2}$ & & 1 & $T_{S}$ & -2 & 0 \\
$S^{2} Q$ & & $T_{S}$ & $f$ & 1 & $\frac{1}{2}$ \\
$S^{2}$ & & $T_{S}$ & 1 & 2 & $\frac{1}{2}$ \\
$S^{2} Q^{3}$ & & 1 & $\bar{f}$ & -1 & $\frac{1}{2}$ \\
$S^{2} Q^{2}$ & & 1 & $T_{A}$ & 0 & $\frac{1}{2}$ \\
$S^{4} Q^{3}$ & & 1 & $\bar{f}$ & 1 & 1 \\
$S^{2} Q^{4}$ & & $T_{S}$ & 1 & -2 & $\frac{1}{2}$ \\
$S^{4}$ & & 1 & 1 & 4 & 1 \\
\hline
\end{tabular}

Corresponding SCIs are

$$
\begin{aligned}
I_{E}= & \frac{(p ; p)_{\infty}^{4}(q ; q)_{\infty}^{4}}{2^{4} 4 !} \prod_{i=1}^{4} \Gamma\left(t_{i}\right) \int_{\mathbb{T}^{4}} \frac{\prod_{i, j=1}^{4} \Gamma\left(t_{i} z_{j}^{ \pm 2}\right) \prod_{i=1}^{2} \Gamma\left(s_{i} z^{ \pm 1}\right)}{\prod_{j=1}^{4} \Gamma\left(z_{j}^{ \pm 2}\right) \prod_{1 \leq j<k \leq 4} \Gamma\left(z_{j}^{ \pm 2} z_{k}^{ \pm 2}\right)} \\
& \times \prod_{i=1}^{2} \prod_{j=1}^{4} \Gamma\left(s_{i}\left(z_{j}^{2} z^{-1}\right)^{ \pm 1}\right) \prod_{i=1}^{2} \prod_{1 \leq j<k \leq 4} \Gamma\left(s_{i} z_{j}^{2} z_{k}^{2} z^{-1}\right) \prod_{j=1}^{4} \frac{d z_{j}}{2 \pi \mathrm{i} z_{j}},
\end{aligned}
$$

where $z=z_{1} z_{2} z_{3} z_{4},\left|s_{i}\right|<1, s^{2} t=\sqrt{p q}$ with $s=\prod_{i=1}^{2} s_{i}, t=\prod_{i=1}^{4} t_{i}$, and

$$
\begin{gathered}
I_{M}=\Gamma\left(s, s t, s^{2}\right) \prod_{i=1}^{2} \Gamma\left(s_{i}^{2}, t s_{i}^{2}\right) \prod_{i=1}^{4} \Gamma\left(t_{i}^{2}, s t_{i}^{2}, s t t_{i}^{-1}, s^{2} t t_{i}^{-1}\right) \\
\times \prod_{i=1}^{2} \prod_{j=1}^{4} \Gamma\left(s_{i}^{2} t_{j}\right) \prod_{1 \leq i<j \leq 4} \Gamma\left(t_{i} t_{j}, s t_{i} t_{j}\right) .
\end{gathered}
$$

3.4. $G=S O(10)$.

3.4.1. $S U(4) \times U(1)$ flavor group. The matter content is [23]

\begin{tabular}{|c|c|c|c|c|}
\hline & $S O(10)$ & $S U(4)$ & $U(1)$ & $U(1)_{R}$ \\
\hline$S$ & $s$ & $f$ & 1 & 0 \\
$Q$ & $f$ & 1 & -8 & 1 \\
\hline \hline$Q^{2}$ & & 1 & -16 & 2 \\
$S^{2} Q$ & & $T_{S}$ & -6 & 1 \\
$S^{4}$ & & $T_{A A S S}$ & 4 & 0 \\
$S^{6} Q$ & & $T_{S}$ & -2 & 1 \\
\hline
\end{tabular}

Corresponding SCIs are

$$
\begin{aligned}
I_{E}= & \frac{(p ; p)_{\infty}^{5}(q ; q)_{\infty}^{5}}{2^{4} 5 !} \int_{\mathbb{T}^{5}} \frac{\prod_{i=1}^{4} \Gamma\left(s_{i} z\right) \prod_{i=1}^{4} \prod_{j=1}^{5} \Gamma\left(s_{i} z_{j}^{2} z^{-1}\right)}{\prod_{1 \leq j<k \leq 5} \Gamma\left(z_{j}^{ \pm 2} z_{k}^{ \pm 2}\right)} \\
& \times \prod_{i=1}^{4} \prod_{1 \leq j<k \leq 5} \Gamma\left(s_{i} z z_{j}^{-2} z_{k}^{-2}\right) \prod_{j=1}^{5} \Gamma\left(t z_{j}^{ \pm 2}\right) \prod_{j=1}^{5} \frac{d z_{j}}{2 \pi \mathrm{i} z_{j}},
\end{aligned}
$$


where $z=z_{1} z_{2} z_{3} z_{4} z_{5},\left|s_{i}\right|,|t|<1$, and

$$
I_{M}=\Gamma\left(t^{2}\right) \Gamma^{2}(s) \prod_{i=1}^{4} \Gamma\left(t s_{i}^{2}, s t s_{i}^{2}\right) \prod_{1 \leq i<j \leq 4} \Gamma\left(t s_{i} s_{j}, s t s_{i} s_{j}, s_{i}^{2} s_{j}^{2}\right) \prod_{i=1}^{4} \prod_{1 \leq j<k \leq 4 ; j, k \neq i} \Gamma\left(s_{i}^{2} s_{j} s_{k}\right)
$$

with $s=\prod_{i=1}^{4} s_{i}$ and the balancing condition $s^{2} t=\sqrt{p q}$. A simple check of the equality of these integrals is obtained in the limit $p=q=0$ and $s_{2,3}=0$.

3.4.2. $S U(3) \times S U(3) \times U(1)$ flavor group. The matter content is [23]

\begin{tabular}{|c|c|c|c|c|c|}
\hline & $S O(10)$ & $S U(3)$ & $S U(3)$ & $U(1)$ & $U(1)_{R}$ \\
\hline$S$ & $s$ & $f$ & 1 & 1 & 0 \\
$Q$ & $f$ & 1 & $f$ & -2 & $\frac{1}{3}$ \\
\hline \hline$Q^{2}$ & & 1 & $T_{S}$ & -4 & $\frac{2}{3}$ \\
$S^{2} Q$ & & $T_{S}$ & $f$ & 0 & $\frac{1}{3}$ \\
$S^{2} Q^{3}$ & & $\bar{f}$ & 1 & -4 & 1 \\
$S^{4}$ & & $\bar{T}_{S}$ & 1 & 4 & 0 \\
$S^{4} Q^{2}$ & & $f$ & $\bar{f}$ & 0 & $\frac{2}{3}$ \\
\hline
\end{tabular}

Corresponding SCIs are

$$
\begin{aligned}
I_{E} & =\frac{(p ; p)_{\infty}^{5}(q ; q)_{\infty}^{5}}{2^{4} 5 !} \int_{\mathbb{T}^{5}} \frac{\prod_{i=1}^{3} \Gamma\left(s_{i} z\right) \prod_{i=1}^{3} \prod_{j=1}^{5} \Gamma\left(s_{i} z_{j}^{2} z^{-1}\right)}{\prod_{1 \leq j<k \leq 5} \Gamma\left(z_{j}^{ \pm 2} z_{k}^{ \pm 2}\right)} \\
& \times \prod_{i=1}^{3} \prod_{1 \leq j<k \leq 5} \Gamma\left(s_{i} z z_{j}^{-2} z_{k}^{-2}\right) \prod_{i=1}^{3} \prod_{j=1}^{5} \Gamma\left(t_{i} z_{j}^{ \pm 2}\right) \prod_{j=1}^{5} \frac{d z_{j}}{2 \pi \mathrm{i} z_{j}},
\end{aligned}
$$

where $z=z_{1} z_{2} z_{3} z_{4} z_{5},\left|s_{i}\right|,\left|t_{j}\right|<1, s^{2} t=\sqrt{p q}$ with $s=\prod_{i=1}^{3} s_{i}, t=\prod_{i=1}^{3} t_{i}$, and

$$
I_{M}=\prod_{i=1}^{3} \Gamma\left(t_{i}^{2}, s^{2} s_{i}^{-2}, s t s_{i}^{-1}\right) \prod_{i, j=1}^{3} \Gamma\left(s_{i}^{2} t_{j}, s t s_{i} t_{j}^{-1}\right) \prod_{1 \leq i<j \leq 3}\left(\Gamma\left(t_{i} t_{j}, s^{2} s_{i}^{-1} s_{j}^{-1}\right) \prod_{k=1}^{3} \Gamma\left(s_{i} s_{j} t_{k}\right)\right) \text {. }
$$

3.4.3. $S U(2) \times S U(5) \times U(1)$ flavor group. The matter content is [23]

\begin{tabular}{|c|c|c|c|c|c|}
\hline & $S O(10)$ & $S U(2)$ & $S U(5)$ & $U(1)$ & $U(1)_{R}$ \\
\hline$S$ & $s$ & $f$ & 1 & 5 & $\frac{1}{4}$ \\
$Q$ & $f$ & 1 & $f$ & -4 & 0 \\
\hline \hline$Q^{2}$ & & 1 & $T_{S}$ & -8 & 0 \\
$S^{2} Q$ & & $T_{S}$ & $f$ & 6 & $\frac{1}{2}$ \\
$S^{2} Q^{3}$ & & 1 & $\bar{T}_{A}$ & -2 & $\frac{1}{2}$ \\
$S^{2} Q^{5}$ & & $T_{S}$ & 1 & -10 & $\frac{1}{2}$ \\
$S^{4}$ & & 1 & $\frac{1}{f}$ & 20 & 1 \\
$S^{4} Q^{4}$ & & 1 & 4 & 1 \\
\hline
\end{tabular}

Corresponding SCIs are

$$
\begin{aligned}
I_{E} & =\frac{(p ; p)_{\infty}^{5}(q ; q)_{\infty}^{5}}{2^{4} 5 !} \int_{\mathbb{T}^{5}} \frac{\prod_{i=1}^{2} \Gamma\left(s_{i} z\right) \prod_{i=1}^{2} \prod_{j=1}^{5} \Gamma\left(s_{i} z_{j}^{2} z^{-1}\right)}{\prod_{1 \leq j<k \leq 5} \Gamma\left(z_{j}^{ \pm 2} z_{k}^{ \pm 2}\right)} \\
& \times \prod_{i=1}^{2} \prod_{1 \leq j<k \leq 5} \Gamma\left(s_{i} z z_{j}^{-2} z_{k}^{-2}\right) \prod_{i, j=1}^{5} \Gamma\left(t_{i} z_{j}^{ \pm 2}\right) \prod_{j=1}^{5} \frac{d z_{j}}{2 \pi \mathrm{i} z_{j}},
\end{aligned}
$$


where $z=z_{1} z_{2} z_{3} z_{4} z_{5},\left|s_{i}\right|,\left|t_{j}\right|<1, s^{2} t=\sqrt{p q}$ with $s=\prod_{i=1}^{2} s_{i}, t=\prod_{i=1}^{5} t_{i}$, and

$$
I_{M}=\Gamma\left(s t, s^{2}\right) \prod_{j=1}^{2} \Gamma\left(t s_{j}^{2}\right) \prod_{i=1}^{5}\left(\Gamma\left(t_{i}^{2}, s t_{i}, s^{2} t t_{i}^{-1}\right) \prod_{j=1}^{2} \Gamma\left(s_{j}^{2} t_{i}\right)\right) \prod_{1 \leq i<j \leq 5} \Gamma\left(t_{i} t_{j}, s t t_{i}^{-1} t_{j}^{-1}\right) .
$$

3.4.4. $S U(3) \times U(1)_{1} \times U(1)_{2}$ flavor group. The matter content is [23]

\begin{tabular}{|c|c|c|c|c|c|}
\hline & $S O(10)$ & $S U(3)$ & $U(1)_{1}$ & $U(1)_{2}$ & $U(1)_{R}$ \\
\hline$S$ & $s$ & $f$ & 1 & 0 & 0 \\
$\bar{S}$ & $c$ & 1 & -3 & 1 & 0 \\
$Q$ & $f$ & 1 & 0 & -2 & 1 \\
\hline \hline$Q^{2}$ & & 1 & 0 & -4 & 2 \\
$S^{2} Q$ & & $T_{S}$ & 2 & -2 & 1 \\
$S \bar{S}$ & & $f$ & -2 & 1 & 0 \\
$S^{3} \bar{S} Q$ & & $T_{A S}$ & 0 & -1 & 1 \\
$S^{2} \bar{S}^{2}$ & & $T_{S}$ & -4 & 2 & 0 \\
$S^{4}$ & & $\bar{T}_{S}$ & 4 & 0 & 0 \\
$S^{5} \bar{S}$ & & $T_{S}$ & 2 & 1 & 0 \\
$S^{4} \bar{S}^{2} Q$ & & $f$ & -2 & 0 & 1 \\
$\bar{S}^{2} Q$ & & 1 & -6 & 0 & 1 \\
$S^{3} \bar{S}^{3} Q^{2}$ & & 1 & -6 & -1 & 2 \\
\hline
\end{tabular}

where $T_{A S}$ stands for the rank three tensor representation which is symmetric in the first two indices and antisymmetric in the last two indices.

Corresponding SCIs are

$$
\begin{aligned}
I_{E} & =\frac{(p ; p)_{\infty}^{5}(q ; q)_{\infty}^{5}}{2^{4} 5 !} \int_{\mathbb{T}^{5}} \frac{\Gamma\left(t z^{-1}\right) \prod_{i=1}^{3}\left(\Gamma\left(s_{i} z\right) \prod_{j=1}^{5} \Gamma\left(s_{i} z_{j}^{2} z^{-1}\right)\right) \prod_{j=1}^{5} \Gamma\left(t z z_{j}^{-2}\right)}{\prod_{1 \leq j<k \leq 5} \Gamma\left(z_{j}^{ \pm 2} z_{k}^{ \pm 2}\right)} \\
& \times \prod_{1 \leq j<k \leq 5}\left(\Gamma\left(t z_{j}^{2} z_{k}^{2} z^{-1}\right) \prod_{i=1}^{3} \Gamma\left(s_{i} z z_{j}^{-2} z_{k}^{-2}\right)\right) \prod_{j=1}^{5} \Gamma\left(u z_{j}^{ \pm 2}\right) \frac{d z_{j}}{2 \pi \mathrm{i} z_{j}},
\end{aligned}
$$

where $z=z_{1} z_{2} z_{3} z_{4} z_{5},\left|s_{i}\right|,|t|,|u|<1, s^{2} t^{2} u=\sqrt{p q}$ with $s=\prod_{i=1}^{3} s_{i}$, and

$$
\begin{aligned}
I_{M}= & \Gamma\left(u^{2}, t^{2} u, s t^{3} u^{2}\right) \Gamma^{2}(s t u) \prod_{i=1}^{3} \Gamma\left(t s_{i}, u s_{i}^{2}, t^{2} s_{i}^{2}, s^{2} s_{i}^{-2}, s t s_{i}^{2}, s t^{2} u s_{i}\right) \\
& \times \prod_{1 \leq i<j \leq 3} \Gamma\left(u s_{i} s_{j}, t^{2} s_{i} s_{j}, s t s_{i} s_{j}, s^{2} s_{i}^{-1} s_{j}^{-1}\right) \prod_{i, j=1 ; i \neq j}^{3} \Gamma\left(t u s_{i}^{2} s_{j}\right) .
\end{aligned}
$$

3.4.5. $S U(2) \times S U(3) \times U(1)_{1} \times U(1)_{2}$ flavor group. The matter content is [23] 


\begin{tabular}{|c|c|c|c|c|c|c|}
\hline & $S O(10)$ & $S U(2)$ & $S U(3)$ & $U(1)_{1}$ & $U(1)_{2}$ & $U(1)_{R}$ \\
\hline$S$ & $s$ & $f$ & 1 & 1 & 1 & 0 \\
$\bar{S}$ & $c$ & 1 & 1 & -2 & 1 & $\frac{1}{2}$ \\
$Q$ & $f$ & 1 & $f$ & 0 & -2 & 0 \\
\hline \hline$Q^{2}$ & & 1 & $T_{S}$ & 0 & -4 & 0 \\
$S^{2} Q$ & & $T_{S}$ & $f$ & 2 & 0 & 0 \\
$\bar{S}^{2} Q$ & & 1 & $f$ & -4 & 0 & 1 \\
$S \bar{S}$ & & $f$ & 1 & -1 & 2 & $\frac{1}{2}$ \\
$S^{2} \bar{S}^{2}$ & & $T_{S}$ & 1 & -2 & 4 & 1 \\
$S^{2} Q^{3}$ & & 1 & 1 & 2 & -4 & 0 \\
$S^{3} \bar{S} Q$ & & $f$ & $f$ & 1 & 2 & $\frac{1}{2}$ \\
$S^{4}$ & & 1 & 1 & 4 & 4 & 0 \\
$S \bar{S} Q^{2}$ & & $f$ & $\bar{f}$ & -1 & -2 & $\frac{1}{2}$ \\
$S^{2} \overline{S^{2}} Q^{2}$ & & 1 & $\frac{f}{f}$ & -2 & 0 & 1 \\
$S^{3} \bar{S} Q^{3}$ & & $f$ & 1 & 1 & -2 & $\frac{1}{2}$ \\
\hline
\end{tabular}

Corresponding SCIs are

$$
\begin{aligned}
I_{E} & =\frac{(p ; p)_{\infty}^{5}(q ; q)_{\infty}^{5}}{2^{4} 5 !} \int_{\mathbb{T}^{5}} \frac{\Gamma\left(t z^{-1}\right) \prod_{i=1}^{2}\left(\Gamma\left(s_{i} z\right) \prod_{j=1}^{5} \Gamma\left(s_{i} z_{j}^{2} z^{-1}\right)\right) \prod_{j=1}^{5} \Gamma\left(t z z_{j}^{-2}\right)}{\prod_{1 \leq j<k \leq 5} \Gamma\left(z_{j}^{ \pm 2} z_{k}^{ \pm 2}\right)} \\
& \times \prod_{1 \leq j<k \leq 5}\left(\Gamma\left(t z_{j}^{2} z_{k}^{2} z^{-1}\right) \prod_{i=1}^{2} \Gamma\left(s_{i} z z_{j}^{-2} z_{k}^{-2}\right)\right) \prod_{j=1}^{5} \Gamma\left(u z_{j}^{ \pm 2}\right) \frac{d z_{j}}{2 \pi \mathrm{i} z_{j}}
\end{aligned}
$$

where $z=z_{1} z_{2} z_{3} z_{4} z_{5},\left|s_{i}\right|,|t|,\left|u_{j}\right|<1, s^{2} t^{2} u=\sqrt{p q}$ with $s=\prod_{i=1}^{3} s_{i}$, and

$$
\begin{gathered}
I_{M}=\Gamma\left(s^{2}, s u, s t^{2}\right) \prod_{i=1}^{2} \Gamma\left(t s_{i}, t^{2} s_{i}^{2}, s t u s_{i}\right) \prod_{i=1}^{3} \Gamma\left(s u_{i}, u_{i}^{2}, t^{2} u_{i}, s t^{2} u u_{i}^{-1}\right) \\
\quad \times \prod_{i=1}^{2} \prod_{j=1}^{3} \Gamma\left(s_{i}^{2} u_{j}, s t s_{i} u_{j}, t u s_{i} u_{j}^{-1}\right) \prod_{1 \leq i<j \leq 3} \Gamma\left(u_{i} u_{j}\right) .
\end{gathered}
$$




\begin{tabular}{|c|c|c|c|c|c|c|}
\hline & $S O(10)$ & $S U(2)_{1}$ & $S U(2)_{2}$ & $U(1)_{1}$ & $U(1)_{2}$ & $U(1)_{R}$ \\
\hline$S$ & $s$ & $f$ & 1 & 1 & 1 & 0 \\
$\bar{S}$ & $c$ & 1 & $f$ & -1 & 1 & 0 \\
$Q$ & $f$ & 1 & 1 & 0 & -8 & 1 \\
\hline \hline$Q^{2}$ & & 1 & 1 & 0 & -16 & 2 \\
$S^{2} Q$ & & $T_{S}$ & 1 & 2 & -6 & 1 \\
$\bar{S}^{2} Q$ & & 1 & $T_{S}$ & -2 & -6 & 1 \\
$S \bar{S}$ & & $f$ & $f$ & 0 & 2 & 0 \\
$S^{4}$ & & 1 & 1 & 4 & 4 & 0 \\
$\bar{S}^{4}$ & & 1 & 1 & -4 & 4 & 0 \\
$S^{2} \bar{S}^{2}$ & & $T_{S}$ & $T_{S}$ & 0 & 4 & 0 \\
$S^{3} \bar{S}^{2}$ & & $f$ & $f$ & 2 & -4 & 1 \\
$S \bar{S}^{3} Q$ & & $f$ & $f$ & -2 & -4 & 1 \\
$S^{2} \bar{S}^{2} Q^{2}$ & & 1 & 1 & 0 & -12 & 2 \\
$S^{4} \bar{S}^{2} Q$ & & $T_{S}$ & 1 & 2 & -2 & 1 \\
$S^{2} \bar{S}^{4} Q$ & & 1 & $T_{S}$ & -2 & -2 & 1 \\
$S^{3} \bar{S}^{3}$ & & $f$ & $f$ & 0 & 6 & 0 \\
$S^{6} \bar{S}^{2}$ & & 1 & 1 & 4 & 8 & 0 \\
$S^{2} \bar{S}^{6}$ & & 1 & 1 & -4 & 8 & 0 \\
\hline
\end{tabular}

Corresponding SCIs are

$$
\begin{aligned}
I_{E}= & \frac{(p ; p)_{\infty}^{5}(q ; q)_{\infty}^{5}}{2^{4} 5 !} \int_{\mathbb{T}^{5}} \frac{\prod_{i=1}^{2} \Gamma\left(s_{i} z, t_{i} z^{-1}\right) \prod_{i=1}^{2} \prod_{j=1}^{5} \Gamma\left(s_{i} z_{j}^{2} z^{-1}, t_{i} z z_{j}^{-2}\right)}{\prod_{1 \leq j<k \leq 5} \Gamma\left(z_{j}^{ \pm 2} z_{k}^{ \pm 2}\right)} \\
& \times \prod_{i=1}^{2} \prod_{1 \leq j<k \leq 5} \Gamma\left(s_{i} z z_{j}^{-2} z_{k}^{-2}, t_{i} z_{j}^{2} z_{k}^{2} z^{-1}\right) \prod_{j=1}^{5} \Gamma\left(u z_{j}^{ \pm 2}\right) \frac{d z_{j}}{2 \pi \mathrm{i} z_{j}},
\end{aligned}
$$

where $z=z_{1} z_{2} z_{3} z_{4} z_{5},\left|s_{i}\right|,\left|t_{i}\right|,|u|<1, s^{2} t^{2} u=\sqrt{p q}$ with $s=\prod_{i=1}^{3} s_{i}$, and

$$
\begin{gathered}
I_{M}=\Gamma\left(s^{2}, t^{2}, u^{2}, s t, s u, t u, s^{3} t, s t^{3}, s t u^{2}, s^{2} t u, s t^{2} u\right) \\
\times \prod_{i=1}^{2} \Gamma\left(u s_{i}^{2}, u t_{i}^{2}, s t_{i}^{2}, t s_{i}^{2}, s t u s_{i}^{2}, s t u t_{i}^{2}\right) \prod_{i, j=1}^{2} \Gamma\left(s_{i} t_{j}, s_{i}^{2} t_{j}^{2}, s u s_{i} t_{j}, t u s_{i} t_{j}, s t s_{i} t_{j}\right) .
\end{gathered}
$$




\begin{tabular}{|c|c|c|c|c|c|}
\hline & $S O(10)$ & $S U(5)$ & $U(1)_{1}$ & $U(1)_{2}$ & $U(1)_{R}$ \\
\hline$S$ & $s$ & 1 & 1 & 5 & $\frac{1}{4}$ \\
$\bar{S}$ & $c$ & 1 & -1 & 5 & $\frac{1}{4}$ \\
$Q$ & $f$ & $f$ & 0 & -4 & 0 \\
\hline \hline$Q^{2}$ & & $T_{S}$ & 0 & -8 & 0 \\
$S^{2} Q$ & & $f$ & 2 & 6 & $\frac{1}{2}$ \\
$\bar{S}^{2} Q$ & & $f$ & -2 & 6 & $\frac{1}{2}$ \\
$S \bar{S}$ & & 1 & 0 & 10 & $\frac{1}{2}$ \\
$S^{2} Q^{5}$ & & 1 & 2 & -10 & $\frac{1}{2}$ \\
$\bar{S}^{2} Q^{5}$ & & 1 & -2 & -10 & $\frac{1}{2}$ \\
$S \bar{S} Q^{2}$ & & $T_{A}$ & 0 & 2 & $\frac{1}{2}$ \\
$S \bar{S} Q^{4}$ & & $\bar{f}$ & 0 & -6 & $\frac{1}{2}$ \\
$S^{2} \bar{S}^{2}$ & & 1 & 0 & 20 & 1 \\
$S^{2} \bar{S}^{2} Q^{4}$ & & $\bar{f}$ & 0 & 4 & 1 \\
\hline
\end{tabular}

Corresponding SCIs are

$$
\begin{aligned}
I_{E}= & \frac{(p ; p)_{\infty}^{5}(q ; q)_{\infty}^{5}}{2^{4} 5 !} \int_{\mathbb{T}^{5}} \frac{\Gamma\left(s z, t z^{-1}\right) \prod_{j=1}^{5} \Gamma\left(s z_{j}^{2} z^{-1}\right)}{\prod_{1 \leq j<k \leq 5} \Gamma\left(z_{j}^{ \pm 2} z_{k}^{ \pm 2}\right)} \\
& \times \prod_{1 \leq j<k \leq 5} \Gamma\left(s z z_{j}^{-2} z_{k}^{-2}, t z_{j}^{2} z_{k}^{2} z^{-1}\right) \prod_{i, j=1}^{5} \Gamma\left(u_{i} z_{j}^{ \pm 2}\right) \prod_{j=1}^{5} \Gamma\left(t z z_{j}^{-2}\right) \frac{d z_{j}}{2 \pi \mathrm{i} z_{j}}
\end{aligned}
$$

where $z=z_{1} z_{2} z_{3} z_{4} z_{5},|s|,|t|,\left|u_{i}\right|<1, s^{2} t^{2} u=\sqrt{p q}$ with $u=\prod_{i=1}^{5} u_{i}$, and

$$
I_{M}=\Gamma\left(s t, s u, t u, s^{2} t^{2}\right) \prod_{i=1}^{5} \Gamma\left(u_{i}^{2}, s^{2} u_{i}, t^{2} u_{i}, s t u u_{i}^{-1}, s^{2} t^{2} u u_{i}^{-1}\right) \prod_{1 \leq i<j \leq 5} \Gamma\left(u_{i} u_{j}, s t u_{i} u_{j}\right) \text {. }
$$

3.5. $G=S O(11)$.

3.5.1. $S U(6) \times U(1)$ flavor group. The matter content is [23]

\begin{tabular}{|c|c|c|c|c|}
\hline & $S O(11)$ & $S U(6)$ & $U(1)$ & $U(1)_{R}$ \\
\hline$S$ & $s$ & 1 & 3 & $\frac{1}{4}$ \\
$Q$ & $f$ & $f$ & -2 & 0 \\
\hline \hline$Q^{2}$ & & $T_{S}$ & -4 & 0 \\
$S^{2} Q^{2}$ & & $T_{A}$ & 2 & $\frac{1}{2}$ \\
$S^{2} Q^{5}$ & & $\bar{f}$ & -4 & $\frac{1}{2}$ \\
$S^{4}$ & & $\frac{1}{f}$ & 12 & 1 \\
$S^{4} Q^{5}$ & & $\frac{1}{2}$ & 1 \\
$S^{2} Q$ & & $f$ & 4 & $\frac{1}{2}$ \\
$S^{2} Q^{6}$ & & 1 & -6 & $\frac{1}{2}$ \\
\hline
\end{tabular}

Corresponding SCIs are

$$
\begin{gathered}
I_{E}=\frac{(p ; p)_{\infty}^{5}(q ; q)_{\infty}^{5}}{2^{5} 5 !} \prod_{i=1}^{6} \Gamma\left(t_{i}\right) \int_{\mathbb{T}^{5}} \frac{\Gamma\left(s z^{ \pm 1}\right) \prod_{j=1}^{5} \Gamma\left(s\left(z_{j}^{2} z^{-1}\right)^{ \pm 1}\right)}{\prod_{j=1}^{5} \Gamma\left(z_{j}^{ \pm 2}\right) \prod_{1 \leq j<k \leq 5} \Gamma\left(z_{j}^{ \pm 2} z_{k}^{ \pm 2}\right)} \\
\times \prod_{1 \leq j<k \leq 5} \Gamma\left(s\left(z z_{j}^{-2} z_{k}^{-2}\right)^{ \pm 1}\right) \prod_{i=1}^{6} \prod_{j=1}^{5} \Gamma\left(t_{i} z_{j}^{ \pm 2}\right) \prod_{j=1}^{5} \frac{d z_{j}}{2 \pi \mathrm{i} z_{j}}
\end{gathered}
$$


where $z=z_{1} z_{2} z_{3} z_{4} z_{5},|s|,\left|t_{i}\right|<1, s^{4} t=\sqrt{p q}$ with $t=\prod_{i=1}^{6} t_{i}$, and

$$
I_{M}=\Gamma\left(s^{4}, s^{2} t\right) \prod_{i=1}^{6} \Gamma\left(s^{2} t_{i}, t_{i}^{2}, s^{2} t t_{i}^{-1}, s^{4} t t_{i}^{-1}\right) \prod_{1 \leq i<j \leq 6} \Gamma\left(t_{i} t_{j}, s^{2} t_{i} t_{j}\right) .
$$

3.5.2. $S U(2)_{1} \times S U(2)_{2} \times U(1)$ flavor group. This $s$-confining theory was found in [81. The matter content is

\begin{tabular}{|c|c|c|c|c|c|}
\hline & $S O(11)$ & $S U(2)_{1}$ & $S U(2)_{2}$ & $U(1)$ & $U(1)_{R}$ \\
\hline$S$ & $s$ & $f$ & 1 & 1 & 0 \\
$Q$ & $f$ & 1 & $f$ & -4 & $\frac{1}{2}$ \\
\hline \hline$Q^{2}$ & & 1 & $T_{S}$ & -8 & 1 \\
$S^{2} Q^{2}$ & & $T_{S}$ & 1 & -6 & 1 \\
$S^{2} Q$ & & $T_{S}$ & $f$ & -2 & $\frac{1}{2}$ \\
$S^{2}$ & & 1 & 1 & 2 & 0 \\
$S^{4}$ & & $T_{4 S}$ & 1 & 4 & 0 \\
$S^{4^{\prime}}$ & & 1 & 1 & 4 & 0 \\
$S^{4} Q^{2}$ & & 1 & $T_{S}$ & -4 & 1 \\
$S^{4} Q^{2}$ & & $T_{S}$ & 1 & -4 & 1 \\
$S^{4} Q$ & & $T_{S}$ & $f$ & 0 & $\frac{1}{2}$ \\
$S^{6} Q^{2}$ & & $T_{S}$ & 1 & -2 & 1 \\
$S^{6} Q$ & & $T_{S}$ & $f$ & 2 & $\frac{1}{2}$ \\
$S^{8}$ & & 1 & 1 & 8 & 0 \\
$S^{8} Q$ & & 1 & $f$ & 4 & $\frac{1}{2}$ \\
$S^{4} Q$ & & 1 & $f$ & 0 & $\frac{1}{2}$ \\
$S^{6}$ & & 1 & 1 & 6 & 0 \\
\hline
\end{tabular}

Here $T_{4 S}$ denotes the totally symmetric tensor of the fourth rank.

Corresponding SCIs are

$$
\begin{gathered}
I_{E}=\frac{(p ; p)_{\infty}^{5}(q ; q)_{\infty}^{5}}{2^{5} 5 !} \prod_{i=1}^{2} \Gamma\left(t_{i}\right) \int_{\mathbb{T}^{5}} \frac{\prod_{i=1}^{2} \Gamma\left(s_{i} z^{ \pm 1}\right) \prod_{i=1}^{2} \prod_{j=1}^{5} \Gamma\left(s_{i}\left(z_{j}^{2} z^{-1}\right)^{ \pm 1}\right)}{\prod_{j=1}^{5} \Gamma\left(z_{j}^{ \pm 2}\right) \prod_{1 \leq j<k \leq 5} \Gamma\left(z_{j}^{ \pm 2} z_{k}^{ \pm 2}\right)} \\
\times \prod_{i=1}^{2} \prod_{1 \leq j<k \leq 5} \Gamma\left(s_{i}\left(z z_{j}^{-2} z_{k}^{-2}\right)^{ \pm 1}\right) \prod_{i=1}^{2} \prod_{j=1}^{5} \Gamma\left(t_{i} z_{j}^{ \pm 2}\right) \prod_{j=1}^{5} \frac{d z_{j}}{2 \pi \mathrm{i} z_{j}},
\end{gathered}
$$

where $z=z_{1} z_{2} z_{3} z_{4} z_{5},|s|,\left|t_{i}\right|<1, s^{4} t=\sqrt{p q}$ with $s=\prod_{i=1}^{2} s_{i}, t=\prod_{i=1}^{2} t_{i}$, and

$$
\begin{aligned}
& I_{M}=\Gamma\left(s, t, s t, s^{3}, s^{3} t, s^{4}\right) \Gamma^{2}\left(s, s^{2} t\right) \prod_{i=1}^{2} \Gamma\left(t_{i}^{2}, s t_{i}, t s_{i}^{2}, s^{2} t_{i}, s s_{i}^{2}\right) \\
& \times \prod_{i=1}^{2} \Gamma\left(s^{2} t_{i}, s^{2} t_{i}^{2}, s t s_{i}^{2}, s^{2} t s_{i}^{2}, s^{3} t_{i}, s_{i}^{4}, s^{4} t_{i}\right) \prod_{i, j=1}^{2} \Gamma\left(s_{i}^{2} t_{j}, s s_{i}^{2} t_{j}, s^{2} s_{i}^{2} t_{j}\right) .
\end{aligned}
$$

3.6. $G=S O(12)$.

3.6.1. $S U(7) \times U(1)$ flavor group. The matter content is [23] 


\begin{tabular}{|c|c|c|c|c|}
\hline & $S O(12)$ & $S U(7)$ & $U(1)$ & $U(1)_{R}$ \\
\hline$S$ & $s$ & 1 & 7 & $\frac{1}{4}$ \\
$Q$ & $f$ & $f$ & -4 & 0 \\
\hline \hline$Q^{2}$ & & $T_{S}$ & -8 & 0 \\
$S^{2} Q^{2}$ & & $T_{A}$ & 6 & $\frac{1}{2}$ \\
$S^{2} Q^{6}$ & & $\bar{f}$ & -10 & $\frac{1}{2}$ \\
$S^{4}$ & & 1 & 28 & 1 \\
$S^{4} Q^{6}$ & & $\frac{1}{f}$ & 4 & 1 \\
\hline
\end{tabular}

Corresponding SCIs are

$$
\begin{array}{r}
I_{E}=\frac{(p ; p)_{\infty}^{6}(q ; q)_{\infty}^{6}}{2^{5} 6 !} \int_{\mathbb{T}^{6}} \frac{\Gamma\left(s z^{ \pm 1}\right)}{\prod_{1 \leq j<k \leq 6} \Gamma\left(z_{j}^{ \pm 2} z_{k}^{ \pm 2}\right)} \\
\times \prod_{1 \leq j<k \leq 6} \Gamma\left(s\left(z z_{j}^{-2} z_{k}^{-2}\right)^{ \pm 1}\right) \prod_{i=1}^{7} \prod_{j=1}^{6} \Gamma\left(t_{i} z_{j}^{ \pm 2}\right) \prod_{j=1}^{6} \frac{d z_{j}}{2 \pi \mathrm{i} z_{j}}
\end{array}
$$

where $z=z_{1} z_{2} z_{3} z_{4} z_{5} z_{6},|s|,\left|t_{i}\right|<1, s^{4} t=\sqrt{p q}$ with $t=\prod_{i=1}^{7} t_{i}$, and

$$
I_{M}=\Gamma\left(s^{4}\right) \prod_{i=1}^{7} \Gamma\left(t_{i}^{2}, s^{2} t t_{i}^{-1}, s^{4} t t_{i}^{-1}\right) \prod_{1 \leq i<j \leq 6} \Gamma\left(t_{i} t_{j}, s^{2} t_{i} t_{j}\right)
$$

3.6.2. $S U(2) \times S U(3) \times U(1)$ flavor group. The matter content is [23]

\begin{tabular}{|c|c|c|c|c|c|}
\hline & $S O(12)$ & $S U(2)$ & $S U(3)$ & $U(1)$ & $U(1)_{R}$ \\
\hline$S$ & $s$ & $f$ & 1 & 3 & $\frac{1}{8}$ \\
$Q$ & $f$ & 1 & $f$ & -8 & 0 \\
\hline \hline$Q^{2}$ & & 1 & $T_{S}$ & -16 & 0 \\
$S^{2}$ & & 1 & $\frac{1}{f}$ & 6 & $\frac{1}{4}$ \\
$S^{2} Q^{2}$ & & $T_{S}$ & -10 & $\frac{1}{4}$ \\
$S^{4}$ & & $T_{4 S}$ & 1 & 12 & $\frac{1}{2}$ \\
$S^{4} Q^{2}$ & & 1 & $T_{S}$ & -4 & $\frac{1}{2}$ \\
$S^{4} Q^{2}$ & & $T_{S}$ & $\frac{1}{2}$ & -4 & $\frac{1}{2}$ \\
$S^{6}$ & & 1 & 1 & 18 & $\frac{3}{4}$ \\
$S^{6} Q^{2}$ & & $T_{S}$ & $\frac{1}{4}$ & 2 & $\frac{3}{4}$ \\
$S^{8} Q^{2}$ & & 1 & $T_{S}$ & 8 & 1 \\
\hline
\end{tabular}

Corresponding SCIs are

$$
\begin{gathered}
I_{E}=\frac{(p ; p)_{\infty}^{6}(q ; q)_{\infty}^{6}}{2^{5} 6 !} \int_{\mathbb{T}^{6}} \frac{\prod_{i=1}^{2} \Gamma\left(s_{i} z^{ \pm 1}\right)}{\prod_{1 \leq j<k \leq 6} \Gamma\left(z_{j}^{ \pm 2} z_{k}^{ \pm 2}\right)} \\
\times \prod_{i=1}^{2} \prod_{1 \leq j<k \leq 6} \Gamma\left(s_{i}\left(z z_{j}^{-2} z_{k}^{-2}\right)^{ \pm 1}\right) \prod_{i=1}^{3} \prod_{j=1}^{6} \Gamma\left(t_{i} z_{j}^{ \pm 2}\right) \prod_{j=1}^{6} \frac{d z_{j}}{2 \pi \mathrm{i} z_{j}}
\end{gathered}
$$


where $z=z_{1} z_{2} z_{3} z_{4} z_{5} z_{6},\left|s_{i}\right|,\left|t_{i}\right|<1, s^{4} t=\sqrt{p q}$ with $s=\prod_{i=1}^{2} s_{i}, t=\prod_{i=1}^{3} t_{i}$, and

$$
\begin{aligned}
I_{M}= & \Gamma\left(s, s^{2}, s^{3}\right) \prod_{i=1}^{2} \Gamma\left(s s_{i}^{2}, s_{i}^{4}\right) \prod_{i=1}^{3} \Gamma\left(t_{i}^{2}, s^{2} t_{i}^{2}, s t t_{i}^{-1}, s^{2} t t_{i}^{-1}, s^{4} t_{i}^{2}, s^{3} t t_{i}^{-1}\right) \\
& \times \prod_{i=1}^{2} \prod_{j=1}^{3} \Gamma\left(s t s_{i}^{2} t_{j}^{-1}, s^{2} t s_{i}^{2} t_{j}^{-1}, t s_{i}^{2} t_{j}^{-1}\right) \prod_{1 \leq i<j \leq 3} \Gamma\left(t_{i} t_{j}, s^{2} t_{i} t_{j}, s^{4} t_{i} t_{j}\right) .
\end{aligned}
$$

3.6.3. $S U(3) \times U(1)_{1} \times U(1)_{2}$ flavor group. The matter content is [23]

\begin{tabular}{|c|c|c|c|c|c|}
\hline & $S O(12)$ & $S U(3)$ & $U(1)_{1}$ & $U(1)_{2}$ & $U(1)_{R}$ \\
\hline$S$ & $s$ & 1 & 1 & 3 & $\frac{1}{8}$ \\
$S^{\prime}$ & $c$ & 1 & -1 & 3 & $\frac{1}{8}$ \\
$Q$ & $f$ & $f$ & 0 & -8 & 0 \\
\hline \hline$Q^{2}$ & & $T_{S}$ & 0 & -16 & 0 \\
$S S^{\prime} Q^{3}$ & & $\frac{1}{f}$ & 0 & -18 & $\frac{1}{4}$ \\
$S^{2} Q^{2}$ & & $\frac{f}{f}$ & -2 & -10 & $\frac{1}{4}$ \\
$S^{\prime 2} Q^{2}$ & & $f$ & 0 & -2 & $\frac{1}{4}$ \\
$S S^{\prime} Q$ & & 1 & 4 & 12 & $\frac{1}{4}$ \\
$S^{4}$ & & 1 & -4 & 12 & $\frac{1}{2}$ \\
$S^{\prime 4}$ & & 1 & 0 & 12 & $\frac{1}{2}$ \\
$S^{2} S^{\prime 2}$ & & 1 & 2 & -12 & $\frac{1}{2}$ \\
$S^{3} S^{\prime} Q^{3}$ & & 1 & -2 & -12 & $\frac{1}{2}$ \\
$S S^{\prime 3} Q^{3}$ & & $\frac{1}{S}$ & 0 & -4 & $\frac{1}{2}$ \\
$S^{2} S^{\prime 2} Q^{2}$ & & $\frac{T}{f}$ & 0 & -4 & $\frac{1}{2}$ \\
$S^{2} S^{\prime 2} Q^{2}$ & & $f$ & 2 & 4 & $\frac{1}{2}$ \\
$S^{3} S^{\prime} Q$ & & $f$ & -2 & 4 & $\frac{1}{2}$ \\
$S S^{\prime 3} Q^{2}$ & & & 0 & -6 & $\frac{3}{4}$ \\
$S^{3} S^{\prime 3} Q^{3}$ & & 1 & 0 & 10 & $\frac{3}{4}$ \\
$S^{3} S^{\prime 3} Q$ & & & $f$ & $\frac{3}{4}$ \\
$S^{4} S^{\prime 2} Q^{2}$ & & $\frac{f}{f}$ & -2 & 2 & $\frac{3}{4}$ \\
$S^{2} S^{\prime 4} Q^{2}$ & & $\frac{1}{f}$ & 0 & 8 & 1 \\
$S^{4} S^{\prime 4}$ & & & 0 & 24 & 1 \\
$S^{4} S^{\prime 4} Q^{2}$ & & $\frac{1}{f}$ & & & \\
\hline
\end{tabular}

Corresponding SCIs are

$$
\begin{array}{r}
I_{E}=\frac{(p ; p)_{\infty}^{6}(q ; q)_{\infty}^{6}}{2^{5} 6 !} \int_{\mathbb{T}^{6}} \frac{\Gamma\left(s z^{ \pm 1}\right)}{\prod_{1 \leq j<k \leq 6} \Gamma\left(z_{j}^{ \pm 2} z_{k}^{ \pm 2}\right)} \prod_{1 \leq j<k \leq 6} \Gamma\left(s\left(z z_{j}^{-2} z_{k}^{-2}\right)^{ \pm 1}\right) \\
\times \prod_{i=1}^{6} \Gamma\left(t\left(z_{i}^{2} z^{-1}\right)^{ \pm 1}\right) \prod_{1 \leq i<j<k \leq 6} \Gamma\left(t\left(z_{i}^{2} z_{j}^{2} z_{k}^{2} z^{-1}\right)^{ \pm 1}\right) \prod_{i=1}^{3} \prod_{j=1}^{6} \Gamma\left(u_{i} z_{j}^{ \pm 2}\right) \prod_{j=1}^{6} \frac{d z_{j}}{2 \pi \mathrm{i} z_{j}}
\end{array}
$$


where $z=z_{1} z_{2} z_{3} z_{4} z_{5} z_{6},|s|,|t|,\left|u_{i}\right|<1,(s t)^{4} u=\sqrt{p q}$ with $u=\prod_{i=1}^{3} u_{i}$, and

$$
\begin{aligned}
I_{M}=\Gamma\left(s t u, s^{4}, t^{4}, s^{2} t^{2}, s t^{3} u, s^{3} t u, s^{3} t^{3} u, s^{4} t^{4}\right) \prod_{1 \leq i<j \leq 3} \Gamma\left(u_{i} u_{j}, s^{2} t^{2} u_{i} u_{j}\right) \\
\quad \times \prod_{i=1}^{3} \Gamma\left(s^{3} t u_{i}, s t^{3} u_{i}, s^{3} t^{3} u_{i}, s^{4} t^{2} u u_{i}^{-1}, s^{2} t^{4} u u_{i}^{-1}, s^{4} t^{4} u u_{i}^{-1}\right) \\
\quad \times \prod_{i=1}^{3} \Gamma\left(u_{i}^{2}, s^{2} u u_{i}^{-1}, t^{2} u u_{i}^{-1}, s t u_{i}, s^{2} t^{2} u_{i}^{2}, s^{2} t^{2} u u_{i}^{-1}\right)
\end{aligned}
$$

3.7. $G=S O(13)$.

3.7.1. $S U(4) \times U(1)$ flavor group. The matter content is [23]

\begin{tabular}{|c|c|c|c|c|}
\hline & $S O(13)$ & $S U(4)$ & $U(1)$ & $U(1)_{R}$ \\
\hline$S$ & $s$ & 1 & 1 & $\frac{1}{8}$ \\
$Q$ & $f$ & $f$ & -2 & 0 \\
\hline \hline$Q^{2}$ & & $T_{S}$ & -4 & 0 \\
$S^{2} Q^{3}$ & & $\frac{1}{4}$ & -4 & $\frac{1}{4}$ \\
$S^{2} Q^{2}$ & & $T_{A}$ & -2 & $\frac{1}{4}$ \\
$S^{4} Q^{4}$ & & $\frac{1}{f}$ & -4 & $\frac{1}{2}$ \\
$S^{4} Q^{3}$ & & $\frac{1}{2}$ & $\frac{1}{2}$ \\
$S^{4} Q^{2}$ & & $T_{S}$ & 0 & $\frac{1}{2}$ \\
$S^{4} Q$ & & $f$ & 2 & $\frac{1}{2}$ \\
$S^{4}$ & & 1 & 4 & $\frac{1}{2}$ \\
$S^{6} Q^{3}$ & & $\frac{1}{f}$ & 0 & $\frac{3}{4}$ \\
$S^{6} Q^{2}$ & & $T_{A}$ & 2 & $\frac{3}{4}$ \\
$S^{8} Q^{3}$ & & $\frac{f}{f}$ & 2 & 1 \\
$S^{8}$ & & 1 & 8 & 1 \\
\hline
\end{tabular}

Corresponding SCIs are

$$
\begin{gathered}
I_{E}=\frac{(p ; p)_{\infty}^{6}(q ; q)_{\infty}^{6}}{2^{6} 6 !} \prod_{i=1}^{4} \Gamma\left(t_{i}\right) \int_{\mathbb{T}^{6}} \frac{\Gamma\left(s z^{ \pm 1}\right) \prod_{j=1}^{6} \Gamma\left(s\left(z_{j}^{2} z^{-1}\right)^{ \pm 1}\right)}{\prod_{j=1}^{6} \Gamma\left(z_{j}^{ \pm 2}\right) \prod_{1 \leq j<k \leq 6} \Gamma\left(z_{j}^{ \pm 2} z_{k}^{ \pm 2}\right)} \\
\times \prod_{1 \leq j<k \leq 6} \Gamma\left(s\left(z z_{j}^{-2} z_{k}^{-2}\right)^{ \pm 1}\right) \prod_{1 \leq i<j<k \leq 6} \Gamma\left(s z_{i}^{2} z_{j}^{2} z_{k}^{2} z^{-1}\right) \prod_{i=1}^{4} \prod_{j=1}^{6} \Gamma\left(t_{i} z_{j}^{ \pm 2}\right) \prod_{j=1}^{6} \frac{d z_{j}}{2 \pi \mathrm{i} z_{j}}
\end{gathered}
$$

where $z=z_{1} z_{2} z_{3} z_{4} z_{5} z_{6},|s|,\left|t_{i}\right|<1, s^{8} t=\sqrt{p q}$ with $t=\prod_{i=1}^{4} t_{i}$, and

$$
\begin{gathered}
I_{M}=\Gamma\left(s^{4}, s^{4} t, s^{8}\right) \prod_{i=1}^{4} \Gamma\left(t_{i}^{2}, s^{2} t t_{i}^{-1}, s^{4} t t_{i}^{-1}, s^{4} t_{i}^{2}, s^{4} t_{i}, s^{6} t t_{i}^{-1}, s^{8} t t_{i}^{-1}\right) \\
\times \prod_{1 \leq i<j \leq 4} \Gamma\left(t_{i} t_{j}, s^{2} t_{i} t_{j}, s^{4} t_{i} t_{j}, s^{6} t_{i} t_{j}\right) .
\end{gathered}
$$

3.8. $G=S O(14)$. 
3.8.1. $S U(5) \times U(1)$ flavor group. The matter content is [23]

\begin{tabular}{|c|c|c|c|c|}
\hline & $S O(14)$ & $S U(5)$ & $U(1)$ & $U(1)_{R}$ \\
\hline$S$ & $s$ & 1 & 5 & $\frac{1}{8}$ \\
$Q$ & $f$ & $f$ & -8 & 0 \\
\hline \hline$Q^{2}$ & & $T_{S}$ & -16 & 0 \\
$S^{2} Q^{3}$ & & $\bar{T}_{A}$ & -14 & $\frac{1}{4}$ \\
$S^{4} Q^{2}$ & & $T_{S}$ & 4 & $\frac{1}{2}$ \\
$S^{4} Q^{4}$ & & $\frac{f}{2}$ & -12 & $\frac{1}{2}$ \\
$S^{6} Q^{3}$ & & $\bar{T}_{A}$ & 6 & $\frac{3}{4}$ \\
$S^{8}$ & & $\frac{1}{f}$ & 40 & 1 \\
$S^{8} Q^{4}$ & & $\frac{}{f}$ & 1 \\
\hline
\end{tabular}

Corresponding SCIs are

$$
\begin{gathered}
I_{E}=\frac{(p ; p)_{\infty}^{7}(q ; q)_{\infty}^{7}}{2^{6} 7 !} \int_{\mathbb{T}^{7}} \frac{\Gamma(s z) \prod_{j=1}^{7} \Gamma\left(s z_{j}^{2} z^{-1}\right)}{\prod_{1 \leq j<k \leq 7} \Gamma\left(z_{j}^{ \pm 2} z_{k}^{ \pm 2}\right)} \\
\times \prod_{1 \leq j<k \leq 7} \Gamma\left(s z z_{j}^{-2} z_{k}^{-2}\right) \prod_{1 \leq i<j<k \leq 7} \Gamma\left(s z_{i}^{2} z_{j}^{2} z_{k}^{2} z^{-1}\right) \prod_{i=1}^{5} \prod_{j=1}^{7} \Gamma\left(t_{i} z_{j}^{ \pm 2}\right) \prod_{j=1}^{7} \frac{d z_{j}}{2 \pi \mathrm{i} z_{j}}
\end{gathered}
$$

where $z=z_{1} z_{2} z_{3} z_{4} z_{5} z_{6} z_{7},|s|,\left|t_{i}\right|<1, s^{8} t=\sqrt{p q}$ with $t=\prod_{i=1}^{5} t_{i}$, and

$$
I_{M}=\Gamma\left(s^{8}\right) \prod_{i=1}^{5} \Gamma\left(t_{i}^{2}, s^{4} t t_{i}^{-1}, s^{8} t t_{i}^{-1}, s^{4} t_{i}^{2}\right) \prod_{1 \leq i<j \leq 5} \Gamma\left(t_{i} t_{j}, s^{2} t t_{i}^{-1} t_{j}^{-1}, s^{4} t_{i} t_{j}, s^{6} t t_{i}^{-1} t_{j}^{-1}\right) .
$$

To summarize, the formulas of this section lead to conjectures for exact evaluations of certain EHIs on $B_{N}$ and $D_{N}$ root systems constructed from the characters of various representations necessarily including the spinor representation, which require now rigorous mathematical proofs.

\section{SELF-DUAL THEORIES With THE SPINOR MATter}

We start by presenting a basic example of the self-dual $\mathcal{N}=1 \mathrm{SYM}$ theory based on the orthogonal gauge group with some number of fields in spinor representation. It was considered first in [24], further examples have been described in [33, 72]. First we consider the theory with $S O(8)$ gauge group and the flavor group $S U(4)_{l} \times S U(4)_{r} \times U(1)_{B}$. The matter content of this theory is

\begin{tabular}{|c|c|c|c|c|c|}
\hline & $S O(8)$ & $S U(4)_{l}$ & $S U(4)_{r}$ & $U(1)_{B}$ & $U(1)_{R}$ \\
\hline$S$ & $s$ & $f$ & 1 & 1 & $\frac{1}{4}$ \\
$Q$ & $f$ & 1 & $f$ & -1 & $\frac{1}{4}$ \\
\hline
\end{tabular}

In 24] there were found 5 theories dual to the original electric theory. We reconsidered these theories using SCI technique and found that there are, actually, only 3 dual theories. Other theories have the fields which can be integrated out and, in particular, their contribution to 't Hooft anomaly matching conditions is trivial (none). The matter fields of dual theories are listed below in the table, where the double lines separate dual theories. 


\begin{tabular}{|c|c|c|c|c|c|}
\hline & $S O(8)$ & $S U(4)_{l}$ & $S U(4)_{r}$ & $U(1)_{B}$ & $U(1)_{R}$ \\
\hline$s$ & $s$ & $\bar{f}$ & 1 & 1 & $\frac{1}{4}$ \\
$v$ & $f$ & 1 & $f$ & -1 & $\frac{1}{4}$ \\
$M$ & 1 & $T_{S}$ & 1 & 2 & $\frac{1}{2}$ \\
$N$ & 1 & $T_{S}$ & 1 & -2 & $\frac{3}{2}$ \\
\hline \hline$s$ & $s$ & $f$ & 1 & 1 & $\frac{1}{4}$ \\
$v$ & $f$ & 1 & $\bar{f}$ & -1 & $\frac{1}{4}$ \\
$\widetilde{M}$ & 1 & 1 & $T_{S}$ & -2 & $\frac{1}{2}$ \\
$\widetilde{N}$ & 1 & 1 & $T_{S}$ & 2 & $\frac{3}{2}$ \\
\hline \hline$s$ & $s$ & $\bar{f}$ & 1 & 1 & $\frac{1}{4}$ \\
$v$ & $f$ & 1 & $\bar{f}$ & -1 & $\frac{1}{4}$ \\
$M$ & 1 & $T_{S}$ & 1 & 2 & $\frac{1}{2}$ \\
$N$ & 1 & $T_{S}$ & 1 & -2 & $\frac{3}{2}$ \\
$\widetilde{M}$ & 1 & 1 & $T_{S}$ & -2 & $\frac{1}{2}$ \\
$\widetilde{N}$ & 1 & 1 & $T_{S}$ & 2 & $\frac{3}{2}$ \\
\hline
\end{tabular}

Corresponding SCIs are given by the following expressions

$$
\begin{gathered}
I_{E}=\frac{(p ; p)_{\infty}^{4}(q ; q)_{\infty}^{4}}{2^{3} 4 !} \int_{\mathbb{T}^{4}} \frac{\prod_{i, j=1}^{4} \Gamma\left(s_{i} z_{j}^{ \pm 2}\right)}{\prod_{1 \leq i<j \leq 4} \Gamma\left(z_{i}^{ \pm 2} z_{j}^{ \pm 2}\right)} \\
\times \prod_{i=1}^{4} \Gamma\left(t_{i} Z^{ \pm 1}\right) \prod_{i=1}^{4} \prod_{1 \leq j<k \leq 4} \Gamma\left(t_{i} z_{j}^{2} z_{k}^{2} Z^{-1}\right) \prod_{j=1}^{4} \frac{d z_{j}}{2 \pi \mathrm{i} z_{j}}
\end{gathered}
$$

where $Z=z_{1} z_{2} z_{3} z_{4}$ and the balancing condition reads $\prod_{i=1}^{4} s_{i} t_{i}=p q$. Magnetic SCIs are

$$
\begin{aligned}
I_{M}^{(1)} & =\prod_{i=1}^{4} \Gamma\left(t_{i}^{2}, S t_{i}^{2}\right) \frac{(p ; p)_{\infty}^{4}(q ; q)_{\infty}^{4}}{2^{3} 4 !} \int_{\mathbb{T}^{4}} \frac{\prod_{i, j=1}^{4} \Gamma\left(s_{i} z_{j}^{ \pm 2}\right)}{\prod_{1 \leq i<j \leq 4} \Gamma\left(z_{i}^{ \pm 2} z_{j}^{ \pm 2}\right)} \\
& \times \prod_{i=1}^{4} \Gamma\left(\frac{\sqrt{T}}{t_{i}} Z^{ \pm 1}\right) \prod_{i=1}^{4} \prod_{1 \leq j<k \leq 4} \Gamma\left(\frac{\sqrt{T}}{t_{i}} z_{j}^{2} z_{k}^{2} Z^{-1}\right) \prod_{j=1}^{4} \frac{d z_{j}}{2 \pi \mathrm{i} z_{j}},
\end{aligned}
$$

for the first magnetic theory;

$$
\begin{aligned}
I_{M}^{(2)} & =\prod_{i=1}^{4} \Gamma\left(s_{i}^{2}, T s_{i}^{2}\right) \frac{(p ; p)_{\infty}^{4}(q ; q)_{\infty}^{4}}{2^{3} 4 !} \int_{\mathbb{T}^{4}} \frac{\prod_{i, j=1}^{4} \Gamma\left(\frac{\sqrt{S}}{s_{i}} z_{j}^{ \pm 2}\right)}{\prod_{1 \leq i<j \leq 4} \Gamma\left(z_{i}^{ \pm 2} z_{j}^{ \pm 2}\right)} \\
& \times \prod_{i=1}^{4} \Gamma\left(t_{i} Z^{ \pm 1}\right) \prod_{i=1}^{4} \prod_{1 \leq j<k \leq 4} \Gamma\left(t_{i} z_{j}^{2} z_{k}^{2} Z^{-1}\right) \prod_{j=1}^{4} \frac{d z_{j}}{2 \pi \mathrm{i} z_{j}},
\end{aligned}
$$

for the second magnetic theory;

$$
\begin{aligned}
I_{M}^{(3)}= & \prod_{i=1}^{4} \Gamma\left(s_{i}^{2}, t_{i}^{2}, T s_{i}^{2}, S t_{i}^{2}\right) \frac{(p ; p)_{\infty}^{4}(q ; q)_{\infty}^{4}}{2^{3} 4 !} \int_{\mathbb{T}^{4}} \frac{\prod_{i, j=1}^{4} \Gamma\left(\frac{\sqrt{S}}{s_{i}} z_{j}^{ \pm 2}\right)}{\prod_{1 \leq i<j \leq 4} \Gamma\left(z_{i}^{ \pm 2} z_{j}^{ \pm 2}\right)} \\
& \times \prod_{i=1}^{4} \Gamma\left(\frac{\sqrt{T}}{t_{i}} Z^{ \pm 1}\right) \prod_{i=1}^{4} \prod_{1 \leq j<k \leq 4} \Gamma\left(\frac{\sqrt{T}}{t_{i}} z_{j}^{2} z_{k}^{2} Z^{-1}\right) \prod_{j=1}^{4} \frac{d z_{j}}{2 \pi \mathrm{i} z_{j}},
\end{aligned}
$$

for the third magnetic theory. 
The situation with other self-dual theories is not so clear, e.g. the self-duality of [24, 72] based on $S O(12)$ gauge group with one field in the spinor representation and 8 quarks in the fundamental representation seems to be incorrect. First, the representations and charges of the dual quarks and spinor representation fields are not changed. Second, the fields $M_{4}$ and $M_{8}$ (taken from the second section of [72]) can be integrated out and their contributions to anomalies cancel out leading thus back to the original theory.

\section{Seiberg Dualities for $S O(N)$ Gauge group With the SPinor matter}

5.1. $G=S O(5)$ and $F=S U\left(N_{f}\right) \times S O(4) \times U(1)$. A duality with $S U\left(N_{f}\right) \times S U(4) \times U(1)$ flavor group was studied in [33] with the claim that it can be derived from a more general duality, which we shall consider later in Sect. 5.8. Using SCI technique we show that this statement is incorrect. In our language, the duality of Sect. 5.8 reduces to the duality discussed below which is based on $S O(4)$-flavor subgroup instead of $S U(4)$.

Let us describe the corrected duality from [33]. The electric theory is represented by the following table

\begin{tabular}{|c|c|c|c|c|c|}
\hline & $S O(5)$ & $S U\left(N_{f}\right)$ & $S O(4)$ & $U(1)$ & $U(1)_{R}$ \\
\hline$Q$ & $f$ & $f$ & 1 & -1 & $1-\frac{3}{N_{f}+2}$ \\
$S$ & $s$ & 1 & $f$ & $\frac{N_{f}}{2}$ & $1-\frac{3}{N_{f}+2}$ \\
\hline
\end{tabular}

while the magnetic theory is

\begin{tabular}{|c|c|c|c|c|c|}
\hline & $S U\left(N_{f}\right)$ & $S U\left(N_{f}\right)$ & $S O(5) \simeq S P(4)$ & $U(1)$ & $U(1)_{R}$ \\
\hline$q$ & $\bar{f}$ & $\bar{f}$ & 1 & 1 & $\frac{3}{N_{f}+2}-\frac{1}{N_{f}}$ \\
$q^{\prime}$ & $f$ & 1 & 1 & $-N_{f}$ & $-1+\frac{6}{N_{f}+2}+\frac{1}{N_{f}}$ \\
$w$ & $T_{S}$ & 1 & 1 & 0 & $\frac{2}{N_{f}}$ \\
$t$ & $\bar{f}$ & 1 & $f$ & 0 & $1-\frac{1}{N_{f}}$ \\
$Y$ & 1 & $f$ & 1 & $N_{f}-1$ & $3-\frac{9}{N_{f}+2}$ \\
$M$ & 1 & $T_{S}$ & 1 & -2 & $2-\frac{6}{N_{f}+2}$ \\
$N$ & 1 & 1 & $f$ & $N_{f}$ & $2-\frac{6}{N_{f}+2}$ \\
\hline
\end{tabular}

The indices are

$$
\begin{aligned}
I_{E}=\frac{(p ; p)_{\infty}^{2}(q ; q)_{\infty}^{2}}{2^{2} 2 !} & \prod_{i=1}^{N_{f}} \Gamma\left(s_{i}\right) \int_{\mathbb{T}^{2}} \frac{\Gamma\left(t u_{1}^{ \pm 1} u_{2}^{ \pm 1}\left(z_{1} z_{2}\right)^{ \pm 1}, t u_{1}^{ \pm 1} u_{2}^{ \pm 1}\left(z_{1} z_{2}^{-1}\right)^{ \pm 1}\right)}{\Gamma\left(z_{1}^{ \pm 2} z_{2}^{ \pm 2}, z_{1}^{ \pm 2}, z_{2}^{ \pm 2}\right)} \\
\times & \prod_{i=1}^{N_{f}} \prod_{j=1}^{2} \Gamma\left(s_{i} z_{j}^{ \pm 2}\right) \prod_{j=1}^{2} \frac{d z_{j}}{2 \pi \mathrm{i} z_{j}},
\end{aligned}
$$


where the balancing condition is $s^{2} t^{4}=(p q)^{N_{f}-1}$ with $s=\prod_{i=1}^{N_{f}} s_{i}$, and

$$
\begin{gathered}
I_{M}=\frac{(p ; p)_{\infty}^{N_{f}-1}(q ; q)_{\infty}^{N_{f}-1}}{N_{f} !} \Gamma\left((p q)^{\frac{N_{f}-1}{2}} s^{-1}\right) \prod_{j=1,2} \Gamma\left((p q)^{\frac{N_{f}-1}{2}} s^{-1} u_{j}^{ \pm 1}\right) \\
\times \prod_{1 \leq i<j \leq N_{f}} \Gamma\left(s_{i} s_{j}\right) \prod_{i=1}^{N_{f}} \Gamma\left(s_{i}^{2}\right) \prod_{i=1}^{N_{f}} \Gamma\left((p q)^{\frac{N_{f}-1}{2}} s^{-1} s_{i}\right) \\
\times \int_{\mathbb{T}^{N_{f}-1}} \prod_{1 \leq i<j \leq N_{f}} \frac{\Gamma\left((p q)^{\frac{1}{N_{f}}} y_{i} y_{j}\right)}{\Gamma\left(y_{i} y_{j}^{-1}, y_{i}^{-1} y_{j}\right)} \prod_{i=1}^{N_{f}} \Gamma\left((p q)^{\frac{1}{N_{f}}} y_{i}^{2}\right) \\
\times \prod_{i, j=1}^{N_{f}} \Gamma\left((p q)^{\frac{N_{f}-1}{2 N_{f}}} s_{i}^{-1} y_{j}^{-1}\right) \prod_{j=1}^{N_{f}} \Gamma\left((p q)^{\frac{1}{2}\left(2+\frac{1}{N_{f}}-N_{f}\right)} s y_{j},(p q)^{\frac{N_{f}-1}{2 N_{f}}} y_{j}^{-1}\right) \\
\times \prod_{i=1,2} \prod_{j=1}^{N_{f}} \Gamma\left((p q)^{\frac{N_{f}-1}{2 N_{f}}} u_{i}^{ \pm 1} y_{j}^{-1}\right) \prod_{j=1}^{N_{f}-1} \frac{d y_{j}}{2 \pi \mathrm{i} y_{j}},
\end{gathered}
$$

where $\prod_{j=1}^{N_{f}} y_{j}=1$.

A simple explanation of the inconsistency of the duality of [33] consists in the mismatch of the number of independent fugacities (parameters) in the dual indices, for the $S U(4)$-flavor subgroup there will be an extra parameter in the electric theory in comparison with the magnetic one. In principle, as described in [116], the integrands entering indices may have different number of parameters, but there should be some additional multipliers to the integrals which cancel the contribution of these extra parameters.

5.2. $S O(7)$ gauge group with $N_{f}$ fundamentals. The $\mathcal{N}=1 \mathrm{SYM}$ electric theory described in this section was historically the first model including a matter field in the spinor representation with known dual theory. It was discovered by Pouliot [93] and it is based on $S O(7)$ gauge group with the following matter content

\begin{tabular}{|c|c|c|c|}
\hline & $S O(7)$ & $S U\left(N_{f}\right)$ & $U(1)_{R}$ \\
\hline$Q$ & $s$ & $f$ & $1-\frac{5}{N_{f}}$ \\
\hline
\end{tabular}

where $s$ means the spinor representation. Pouliot found the following dual magnetic theory

\begin{tabular}{|c|c|c|c|}
\hline & $S U\left(N_{f}-4\right)$ & $S U\left(N_{f}\right)$ & $U(1)_{R}$ \\
\hline$q$ & $\bar{f}$ & $\bar{f}$ & $\frac{5}{N_{f}}-\frac{1}{N_{f}-4}$ \\
$w$ & $T_{S}$ & 1 & $\frac{2}{N_{f}-4}$ \\
$M$ & 1 & $T_{S}$ & $2-\frac{10}{N_{f}}$ \\
\hline
\end{tabular}

where the number of flavors is constrained by the conformal window $6 \leq N_{f} \leq 15$.

According to this duality one should have equality of the following SCIs

$$
I_{E}=\frac{(p ; p)_{\infty}^{3}(q ; q)_{\infty}^{3}}{2^{3} 3 !} \int_{\mathbb{T}^{3}} \frac{\prod_{i=1}^{N_{f}} \Gamma\left(t_{i}\left(z_{1} z_{2} z_{3}\right)^{ \pm 1}\right) \prod_{i=1}^{N_{f}} \prod_{j=1}^{3} \Gamma\left(t_{i}\left(\frac{z_{j}^{2}}{z_{1} z_{2} z_{3}}\right)^{ \pm 1}\right)}{\prod_{1 \leq i<j \leq 3} \Gamma\left(z_{i}^{ \pm 2} z_{j}^{ \pm 2}\right) \prod_{j=1}^{3} \Gamma\left(z_{j}^{ \pm 2}\right)} \prod_{j=1}^{3} \frac{d z_{j}}{2 \pi \mathrm{i} z_{j}}
$$


with $|t|,\left|t_{j}\right|<1$, and the balancing condition $\prod_{m=1}^{N_{f}} t_{m}=(p q)^{\left(N_{f}-5\right) / 2}$, and (with $\left.\prod_{j=1}^{N_{f}-4} y_{j}=1\right)$

$$
\begin{aligned}
& I_{M}=\prod_{1 \leq i<j \leq N_{f}} \Gamma\left(t_{i} t_{j}\right) \prod_{j=1}^{N_{f}} \Gamma\left(t_{j}^{2}\right) \frac{(p ; p)_{\infty}^{N_{f}-5}(q ; q)_{\infty}^{N_{f}-5}}{\left(N_{f}-4\right) !} \int_{\mathbb{T}^{N_{f}}-5} \prod_{j=1}^{N_{f}-5} \frac{d y_{j}}{2 \pi \mathrm{i} y_{j}} \\
& \times \prod_{1 \leq i<j \leq N_{f}-4} \frac{\Gamma\left((p q)^{\frac{1}{N_{f}-4}} y_{i} y_{j}\right)}{\Gamma\left(y_{i} y_{j}^{-1}, y_{i}^{-1} y_{j}\right)} \prod_{j=1}^{N_{f}-4} \Gamma\left((p q)^{\frac{1}{N_{f}-4}} y_{j}^{2}\right) \prod_{i=1}^{N_{f}} \Gamma\left(S^{\frac{1}{N_{f}-4}} t_{i}^{-1} y_{j}^{-1}\right) .
\end{aligned}
$$

To stress the non-trivial character of $S O(N)$-dualities with spinor matter and promote them, we describe the duality for $\mathcal{N}=1 \mathrm{SYM}$ theory with the $G_{2}$ gauge group proposed in [93. Pouliot's idea to derive this model consists in the following: $G_{2}$ is a subgroup of $S O(7)$ and the corresponding duality can be obtained from the $S O(7)$-group case with $N_{f}$ fields in the spinor representation after giving masses to some mesons or integrating out one of the quarks. In our language one should calculate accurately the limit $t_{N_{f}} \rightarrow 1$ in the electric and magnetic SCIs. In the magnetic SCI one has the diverging multiplier $\Gamma\left(t_{N_{f}}^{2}\right)$ in front of the integral, which is the only piece of SCI problematic for this limit. Therefore we can plug $t_{N_{f}}=1$ in other terms and see that the rank of the magnetic gauge group is not changed, i.e. no Higgs mechanism applies from the physical point of view.

On the electric side one has a divergency coming from the poles approaching the integration contour and it is necessary to use the residue calculus [25]. Let us slightly deform the contour $\mathbb{T}$ for the 3rd integration variable and pick up the residues of the poles at $z_{3}=t_{N_{f}}\left(z_{1} z_{2}\right)^{ \pm 1}$ and their reciprocals. Now divide both electric and magnetic SCIs by $\Gamma\left(t_{N_{f}}^{2}\right)$ and take the limit $t_{N_{f}} \rightarrow 1$. Then the electric index can be rewritten as integral (13.3) of [117] with $N_{f}$ replaced by $N_{f}-1$ which describes SCI of the $G_{2}$ gauge group theory with $N_{f}-1$ fundamental quarks:

$$
I_{E}=\frac{(p ; p)_{\infty}^{2}(q ; q)_{\infty}^{2}}{2^{2} 3} \prod_{m=1}^{N_{f}-1} \Gamma\left(t_{m}\right) \int_{\mathbb{T}^{2}} \frac{\prod_{k=1}^{3} \prod_{m=1}^{N_{f}-1} \Gamma\left(t_{m} z_{k}^{ \pm 1}\right)}{\prod_{1 \leq j<k \leq 3} \Gamma\left(z_{j}^{ \pm 1} z_{k}^{ \pm 1}\right)} \prod_{k=1}^{2} \frac{d z_{k}}{2 \pi \mathrm{i} z_{k}}
$$

where $z_{1} z_{2} z_{3}=1$ and the balancing condition reads $\prod_{m=1}^{N_{f}-1} t_{m}=(p q)^{\left(N_{f}-5\right) / 2}$. The magnetic index (5.4) reduces to integral (13.4) of [117]:

$$
\begin{gathered}
I_{M}=\frac{(p ; p)_{\infty}^{N_{f}-5}(q ; q)_{\infty}^{N_{f}-5}}{\left(N_{f}-4\right) !} \prod_{1 \leq j<k \leq N_{f}-1} \Gamma\left(t_{j} t_{k}\right) \prod_{j=1}^{N_{f}-1} \Gamma\left(t_{j}^{2}\right) \\
\times \int_{\mathbb{T}^{N_{f}-5}} \prod_{1 \leq j<k \leq N_{f}-4} \frac{\Gamma\left((p q)^{r_{s}} z_{j} z_{k}\right)}{\Gamma\left(z_{j}^{-1} z_{k}, z_{j} z_{k}^{-1}\right)} \prod_{j=1}^{N_{f}-4} \Gamma\left((p q)^{r_{s}} z_{j}^{2}\right) \\
\times \prod_{j=1}^{N_{f}-4} \Gamma\left((p q)^{\left(1-r_{s}\right) / 2} z_{j}^{-1}\right) \prod_{k=1}^{N_{f}-1} \Gamma\left((p q)^{\left(1-r_{s}\right) / 2} t_{k}^{-1} z_{j}^{-1}\right) \prod_{j=1}^{N_{f}-5} \frac{d z_{j}}{2 \pi \mathrm{i} z_{j}}
\end{gathered}
$$

where $\prod_{j=1}^{N_{f}-4} z_{j}=1$. Another possibility of deriving this $G_{2}$-duality out of the standard Seiberg duality for $S U(3)$-gauge group has been described in [117].

5.3. $G=S O(7)$ and $F=S U\left(N_{f}\right) \times U(1)$. The electric theory is represented in the following table [20] 


\begin{tabular}{|c|c|c|c|c|}
\hline & $S O(7)$ & $S U\left(N_{f}\right)$ & $U(1)$ & $U(1)_{R}$ \\
\hline$Q$ & $f$ & $f$ & -1 & $\frac{N_{f}-4}{N_{f}}$ \\
$S$ & $s$ & 1 & $N_{f}$ & 0 \\
\hline
\end{tabular}

while the magnetic theory is

\begin{tabular}{|c|c|c|c|c|}
\hline & $S U\left(N_{f}-3\right)$ & $S U\left(N_{f}\right)$ & $U(1)$ & $U(1)_{R}$ \\
\hline$q$ & $f$ & $\bar{f}$ & $\frac{2 N_{f}-3}{N_{f}-3}$ & $\frac{3\left(N_{f}-4\right)}{N_{f}\left(N_{f}-3\right)}$ \\
$q^{\prime}$ & $f$ & 1 & $\frac{N_{f}}{N_{f}-3}$ & $\frac{N_{f}-4}{N_{f}-3}$ \\
$w$ & $\bar{T}_{S}$ & 1 & $-\frac{2 N_{f}}{N_{f}-3}$ & $\frac{2}{N_{f}-3}$ \\
$M$ & 1 & $T_{S}$ & -2 & $\frac{2\left(N_{f}-4\right)}{N_{f}}$ \\
$L$ & 1 & 1 & $2 N_{f}$ & 0 \\
\hline
\end{tabular}

where $5 \leq N_{f} \leq 13$. For the electric theory we have

$$
\begin{aligned}
I_{E}=\frac{(p ; p)_{\infty}^{3}(q ; q)_{\infty}^{3}}{2^{3} 3 !} & \prod_{i=1}^{N_{f}} \Gamma\left(s_{i}\right) \int_{\mathbb{T}^{3}} \frac{\Gamma\left(t z^{ \pm 1}\right) \prod_{j=1}^{3} \Gamma\left(t\left(z_{j}^{2} z^{-1}\right)^{ \pm 1}\right)}{\prod_{j=1}^{3} \Gamma\left(z_{j}^{ \pm 2}\right) \prod_{1 \leq i<j \leq 3} \Gamma\left(z_{i}^{ \pm 2} z_{j}^{ \pm 2}\right)} \\
& \times \prod_{i=1}^{N_{f}} \prod_{j=1}^{3} \Gamma\left(s_{i} z_{j}^{ \pm 2}\right) \prod_{j=1}^{3} \frac{d z_{j}}{2 \pi \mathrm{i} z_{j}},
\end{aligned}
$$

where $z=z_{1} z_{2} z_{3}$ and the balancing condition reads $s t=(p q)^{\frac{1}{2}\left(N_{f}-4\right)}$ with $s=\prod_{i=1}^{N_{f}} s_{i}$. In the magnetic theory we have (with $\prod_{j=1}^{N_{f}-3} y_{j}=1$ )

$$
\begin{aligned}
I_{M} & =\Gamma\left(t^{2}\right) \prod_{i=1}^{N_{f}} \Gamma\left(s_{i}^{2}\right) \prod_{1 \leq i<j \leq N_{f}} \Gamma\left(s_{i} s_{j}\right) \frac{(p ; p)_{\infty}^{N_{f}-4}(q ; q)_{\infty}^{N_{f}-4}}{\left(N_{f}-3\right) !} \\
\times \int_{\mathbb{T}^{N_{f}-4}} & \prod_{1 \leq i<j \leq N_{f}-3} \frac{\Gamma\left(s^{\frac{2}{\left(N_{f}-4\right)\left(N_{f}-3\right)}} t^{-\frac{2\left(N_{f}-5\right)}{\left(N_{f}-3\right)\left(N_{f}-4\right)}} y_{i}^{-1} y_{j}^{-1}\right)}{\Gamma\left(y_{i} y_{j}^{-1}, y_{i}^{-1} y_{j}\right)} \prod_{i=1}^{N_{f}} \prod_{j=1}^{N_{f}-3} \Gamma\left(\left(s t^{2}\right)^{\frac{1}{N_{f}-3}} s_{i}^{-1} y_{j}\right) \\
& \times \prod_{j=1}^{N_{f}-3} \Gamma\left(\left(s t^{2}\right)^{\frac{1}{N_{f}-3}} y_{j}, s^{\frac{2}{\left(N_{f}-4\right)\left(N_{f}-3\right)}} t^{-\frac{2\left(N_{f}-5\right)}{\left(N_{f}-3\right)\left(N_{f}-4\right)}} y_{i}^{-2}\right) \prod_{j=1}^{N_{f}-4} \frac{d y_{j}}{2 \pi \mathrm{i} y_{j}} .
\end{aligned}
$$

5.4. $G=S O(7)$ and $F=S U\left(N_{f}\right) \times S U(2) \times U(1)$. The electric theory is [20]

\begin{tabular}{|c|c|c|c|c|c|}
\hline & $S O(7)$ & $S U\left(N_{f}\right)$ & $S U(2)$ & $U(1)$ & $U(1)_{R}$ \\
\hline$Q$ & $f$ & $f$ & 1 & -2 & $1-5 / N_{f}$ \\
$S$ & $s$ & 1 & $f$ & $N_{f}$ & 1 \\
\hline
\end{tabular}

while the magnetic theory is 


\begin{tabular}{|c|c|c|c|c|c|}
\hline & $S U\left(N_{f}-2\right)$ & $S U\left(N_{f}\right)$ & $S O(3)$ & $U(1)$ & $U(1)_{R}$ \\
\hline$q$ & $f$ & $\bar{f}$ & 1 & 2 & $\frac{2\left(N_{f}-5\right)}{N_{f}\left(N_{f}-2\right)}$ \\
$q^{\prime}$ & $f$ & 1 & $f$ & 0 & $\frac{N_{f}-3}{N_{f}-2}$ \\
$\widetilde{q}$ & $\bar{f}$ & 1 & 1 & $-2 N_{f}$ & $-\frac{N_{f}-3}{N_{f}-2}$ \\
$w$ & $\bar{T}_{S}$ & 1 & 1 & 0 & $\frac{2}{N_{f}-2}$ \\
$M$ & 1 & $T_{S}$ & 1 & -4 & $2-10 / N_{f}$ \\
$L$ & 1 & 1 & $f$ & $2 N_{f}$ & 2 \\
$N$ & 1 & $f$ & 1 & $2\left(N_{f}-1\right)$ & $3-5 / N_{f}$ \\
\hline
\end{tabular}

where $4 \leq N_{f} \leq 12$. The electric theory SCI is

$$
\begin{aligned}
I_{E}=\frac{(p ; p)_{\infty}^{3}(q ; q)_{\infty}^{3}}{2^{3} 3 !} & \prod_{i=1}^{N_{f}} \Gamma\left(s_{i}\right) \int_{\mathbb{T}^{3}} \frac{\Gamma\left(y x^{ \pm 1} z^{ \pm 1}\right) \prod_{j=1}^{3} \Gamma\left(y x^{ \pm 1}\left(z_{j}^{2} z^{-1}\right)^{ \pm 1}\right)}{\prod_{j=1}^{3} \Gamma\left(z_{j}^{ \pm 2}\right) \prod_{1 \leq i<j \leq 3} \Gamma\left(z_{i}^{ \pm 2} z_{j}^{ \pm 2}\right)} \\
& \times \prod_{i=1}^{N_{f}} \prod_{j=1}^{3} \Gamma\left(s_{i} z_{j}^{ \pm 2}\right) \prod_{j=1}^{3} \frac{d z_{j}}{2 \pi \mathrm{i} z_{j}},
\end{aligned}
$$

where $z=z_{1} z_{2} z_{3}$ and the balancing condition reads $s y^{2}=(p q)^{\frac{1}{2}\left(N_{f}-3\right)}$ with $s=\prod_{i=1}^{N_{f}} s_{i}$. In the magnetic theory we have (with $\prod_{j=1}^{N_{f}-2} y_{j}=1$ )

$$
\begin{aligned}
I_{M} & =\Gamma\left(y^{2}, y^{2} x^{ \pm 1}\right) \prod_{i=1}^{N_{f}} \Gamma\left(s_{i}^{2}, y^{2} s_{i}\right) \prod_{1 \leq i<j \leq N_{f}} \Gamma\left(s_{i} s_{j}\right) \frac{(p ; p)_{\infty}^{N_{f}-3}(q ; q)_{\infty}^{N_{f}-3}}{\left(N_{f}-2\right) !} \\
\times & \int_{\mathbb{T}^{N_{f}}-3} \prod_{1 \leq i<j \leq N_{f}-2} \frac{\Gamma\left((p q)^{\frac{1}{N_{f}-2}} y_{i}^{-1} y_{j}^{-1}\right)}{\Gamma\left(y_{i} y_{j}^{-1}, y_{i}^{-1} y_{j}\right)} \prod_{i=1}^{N_{f}} \prod_{j=1}^{N_{f}-2} \Gamma\left((p q)^{\frac{N_{f}-5}{2\left(N_{f}-2\right)}} s_{i}^{-1} y_{j}\right) \\
& \times \prod_{j=1}^{N_{f}-2} \Gamma\left((p q)^{\frac{1}{N_{f}-2}} y_{j}^{-2},(p q)^{\frac{N_{f}-1}{2\left(N_{f}-2\right)}} y^{-1} y_{j}^{-1},(p q)^{\frac{N_{f}-3}{2\left(N_{f}-2\right)}} y_{j},(p q)^{\frac{N_{f}-3}{2\left(N_{f}-2\right)}} x^{ \pm 1} y_{j}\right) \prod_{j=1}^{N_{f}-3} \frac{d y_{j}}{2 \pi \mathrm{i} y_{j}}
\end{aligned}
$$

5.5. $G=S O(8)$ and $F=S U\left(N_{f}\right) \times U(1)$. The electric theory is 94

\begin{tabular}{|c|c|c|c|c|}
\hline & $S U\left(N_{f}-4\right)$ & $S U\left(N_{f}\right)$ & $U(1)$ & $U(1)_{R}$ \\
\hline$Q$ & $\bar{f}$ & $f$ & $2 N_{f}-4$ & $\frac{6\left(N_{f}-5\right)}{\left(N_{f}+1\right)\left(N_{f}-4\right)}$ \\
$S$ & $T_{S}$ & 1 & $-2 N_{f}$ & $\frac{12}{\left(N_{f}+1\right)\left(N_{f}-4\right)}$ \\
\hline
\end{tabular}

while the magnetic theory is

\begin{tabular}{|c|c|c|c|c|}
\hline & $S O(8)$ & $S U\left(N_{f}\right)$ & $U(1)$ & $U(1)_{R}$ \\
\hline$q$ & $f$ & $\bar{f}$ & $4-N_{f}$ & $\frac{N_{f}-5}{N_{f}+1}$ \\
$p$ & $s$ & 1 & $N_{f}\left(N_{f}-4\right)$ & $\frac{N_{f}-5}{N_{f}+1}$ \\
$M$ & 1 & $T_{S}$ & $2 N_{f}-8$ & $\frac{12}{N_{f}+1}$ \\
$U$ & 1 & 1 & $-2 N_{f}\left(N_{f}-4\right)$ & $\frac{12}{N_{f}+1}$ \\
\hline
\end{tabular}


where $6 \leq N_{f} \leq 16$. The electric theory SCI is

$$
\begin{aligned}
I_{E}= & \frac{(p ; p)_{\infty}^{N_{f}-5}(q ; q)_{\infty}^{N_{f}-5}}{\left(N_{f}-4\right) !} \int_{\mathbb{T}^{N_{f}-5}} \prod_{1 \leq i<j \leq N_{f}-4} \frac{\Gamma\left(u z_{i} z_{j}\right)}{\Gamma\left(z_{i} z_{j}^{-1}, z_{i}^{-1} z_{j}\right)} \\
& \times \prod_{j=1}^{N_{f}-4} \Gamma\left(u z_{j}^{2}\right) \prod_{i=1}^{N_{f}} \prod_{j=1}^{N_{f}-4} \Gamma\left(s_{i} z_{j}^{-1}\right) \prod_{j=1}^{N_{f}-5} \frac{d z_{j}}{2 \pi \mathrm{i} z_{j}},
\end{aligned}
$$

where $\prod_{j=1}^{N_{f}-4} z_{j}=1$ and the balancing condition reads $s u^{N_{f}-2}=(p q)^{3}$ with $s=\prod_{i=1}^{N_{f}} s_{i}$. In the magnetic theory we have (with $z=z_{1} z_{2} z_{3} z_{4}$ )

$$
\begin{aligned}
I_{M} & =\Gamma\left(u^{n-4}\right) \prod_{1 \leq i<j \leq N_{f}} \Gamma\left(u s_{i} s_{j}\right) \prod_{i=1}^{N_{f}} \Gamma\left(u s_{i}^{2}\right) \frac{(p ; p)_{\infty}^{4}(q ; q)_{\infty}^{4}}{2^{3} 4 !} \int_{\mathbb{T}^{4}} \frac{\Gamma\left(s^{\frac{1}{6}} u^{-\frac{1}{3}\left(N_{f}-5\right)} z^{ \pm 1}\right)}{\prod_{1 \leq i<j \leq 4} \Gamma\left(z_{i}^{ \pm 2} z_{j}^{ \pm 2}\right)} \\
& \times \prod_{1 \leq i<j \leq 4} \Gamma\left(s^{\frac{1}{6}} u^{-\frac{1}{3}\left(N_{f}-5\right)} z_{i}^{2} z_{j}^{2} z^{-1}\right) \prod_{i=1}^{N_{f}} \prod_{j=1}^{4} \Gamma\left(s^{\frac{1}{6}} u^{\frac{1}{6}\left(N_{f}-5\right)} s_{i}^{-1} z_{j}^{ \pm 2}\right) \prod_{j=1}^{4} \frac{d z_{j}}{2 \pi \mathrm{i} z_{j}} .
\end{aligned}
$$

5.6. $G=S O(8)$ and $F=S U\left(N_{f}\right) \times U(1)_{1} \times U(1)_{2}$. The electric theory is [20]

\begin{tabular}{|c|c|c|c|c|c|}
\hline & $S O(8)$ & $S U\left(N_{f}\right)$ & $U(1)_{1}$ & $U(1)_{2}$ & $U(1)_{R}$ \\
\hline$Q$ & $f$ & $f$ & -2 & 0 & $1-6 / N_{f}$ \\
$S$ & $s$ & 1 & $N_{f}$ & 1 & 1 \\
$S^{\prime}$ & $c$ & 1 & $N_{f}$ & -1 & 1 \\
\hline
\end{tabular}

while the magnetic theory is

\begin{tabular}{|c|c|c|c|c|c|}
\hline & $S U\left(N_{f}-3\right)$ & $S U\left(N_{f}\right)$ & $U(1)_{1}$ & $U(1)_{2}$ & $U(1)_{R}$ \\
\hline$q$ & $f$ & $\bar{f}$ & 2 & 0 & $\frac{5 N_{f}-18}{N_{f}\left(N_{f}-3\right)}$ \\
$q^{\prime}$ & $f$ & 1 & 0 & 2 & $\frac{N_{f}-4}{N_{f}-3}$ \\
$q^{\prime \prime}$ & $f$ & 1 & 0 & -2 & $\frac{N_{f}-4}{N_{f}-3}$ \\
$\widetilde{q}$ & $\bar{f}$ & 1 & $-2 N_{f}$ & 0 & $-\frac{N_{f}-4}{N_{f}-3}$ \\
$w$ & $\bar{T}_{S}$ & 1 & 0 & 0 & $\frac{2}{N_{f}-3}$ \\
$M$ & 1 & $T_{S}$ & -4 & 0 & $2-12 / N_{f}$ \\
$L_{1}$ & 1 & 1 & $2 N_{f}$ & 2 & 2 \\
$L_{2}$ & 1 & 1 & $2 N_{f}$ & -2 & 2 \\
$N$ & 1 & $f$ & $2\left(N_{f}-1\right)$ & 0 & $3-6 / N_{f}$ \\
\hline
\end{tabular}

where $5 \leq N_{f} \leq 15$. The electric theory SCI is

$$
\begin{aligned}
I_{E} & =\frac{(p ; p)_{\infty}^{4}(q ; q)_{\infty}^{4}}{2^{3} 4 !} \int_{\mathbb{T}^{4}} \frac{\Gamma\left(t z^{ \pm 1}\right) \prod_{1 \leq i<j \leq 4} \Gamma\left(t z_{i}^{2} z_{j}^{2} z^{-1}\right)}{\prod_{1 \leq i<j \leq 4} \Gamma\left(z_{i}^{ \pm 2} z_{j}^{ \pm 2}\right)} \\
& \times \prod_{j=1}^{4} \Gamma\left(u\left(z_{j}^{2} z^{-1}\right)^{ \pm 1}\right) \prod_{i=1}^{N_{f}} \prod_{j=1}^{4} \Gamma\left(s_{i} z_{j}^{ \pm 2}\right) \prod_{j=1}^{4} \frac{d z_{j}}{2 \pi \mathrm{i} z_{j}},
\end{aligned}
$$


where $z=z_{1} z_{2} z_{3} z_{4}$ and the balancing condition reads $s t u=(p q)^{\frac{1}{2}\left(N_{f}-4\right)}$ with $s=\prod_{i=1}^{N_{f}} s_{i}$. In the magnetic theory we have (with $\prod_{j=1}^{N_{f}-3} y_{j}=1$ )

$$
\begin{gathered}
I_{M}=\Gamma\left(u^{2}, t^{2}\right) \prod_{i=1}^{N_{f}} \Gamma\left(s_{i}^{2}, t u s_{i}\right) \prod_{1 \leq i<j \leq N_{f}} \Gamma\left(s_{i} s_{j}\right) \frac{(p ; p)_{\infty}^{N_{f}-4}(q ; q)_{\infty}^{N_{f}-4}}{\left(N_{f}-3\right) !} \\
\times \int_{\mathbb{T}^{N_{f}-4}} \prod_{1 \leq i<j \leq N_{f}-3} \frac{\Gamma\left((p q)^{\frac{1}{N_{f}-3}} y_{i}^{-1} y_{j}^{-1}\right)}{\Gamma\left(y_{i} y_{j}^{-1}, y_{i}^{-1} y_{j}\right)} \prod_{i=1}^{N_{f}} \prod_{j=1}^{N_{f}-3} \Gamma\left((p q)^{\frac{N_{f}-4}{2\left(N_{f}-3\right)}} s_{i}^{-1} y_{j}\right) \\
\times \prod_{j=1}^{N_{f}-3} \Gamma\left((p q)^{\frac{1}{N_{f}-3}} y_{j}^{-2},(p q)^{\frac{N_{f}-2}{2\left(N_{f}-3\right)}}(t u)^{-1} y_{j}^{-1},(p q)^{\frac{N_{f}-4}{2\left(N_{f}-3\right)}}\left(t u^{-1}\right)^{ \pm 1} y_{j}\right) \prod_{j=1}^{N_{f}-4} \frac{d y_{j}}{2 \pi \mathrm{i} y_{j}}
\end{gathered}
$$

5.7. $G=S O(9)$ and $F=S U\left(N_{f}\right) \times U(1)$. The electric theory is [20]

\begin{tabular}{|c|c|c|c|c|}
\hline & $S O(9)$ & $S U\left(N_{f}\right)$ & $U(1)$ & $U(1)_{R}$ \\
\hline$Q$ & $f$ & $f$ & -2 & $1-5 / N_{f}$ \\
$S$ & $s$ & 1 & $N_{f}$ & 0 \\
\hline
\end{tabular}

while the magnetic theory is

\begin{tabular}{|c|c|c|c|c|}
\hline & $S U\left(N_{f}-4\right)$ & $S U\left(N_{f}\right)$ & $U(1)$ & $U(1)_{R}$ \\
\hline$q$ & $f$ & $\bar{f}$ & 2 & $\frac{\left.4\left(N_{f}-5\right)\right)}{N_{f}\left(N_{f}-4\right)}$ \\
$q^{\prime}$ & $f$ & 1 & 0 & $\frac{N_{f}-5}{N_{f}-4}$ \\
$\widetilde{q}$ & $\bar{f}$ & 1 & $-2 N_{f}$ & $\frac{N_{f}-3}{N_{f}-4}$ \\
$w$ & $\bar{T}_{S}$ & 1 & 0 & $\frac{2}{N_{f}-4}$ \\
$M$ & 1 & $T_{S}$ & -4 & $2-10 / N_{f}$ \\
$L$ & 1 & 1 & $2 N_{f}$ & 0 \\
$N$ & 1 & $f$ & $2\left(N_{f}-1\right)$ & $1-5 / N_{f}$ \\
\hline
\end{tabular}

where $6 \leq N_{f} \leq 18$. The electric theory SCI is

$$
\begin{gathered}
I_{E}=\prod_{i=1}^{N_{f}} \Gamma\left(s_{i}\right) \frac{(p ; p)_{\infty}^{4}(q ; q)_{\infty}^{4}}{2^{4} 4 !} \int_{\mathbb{T}^{4}} \frac{\Gamma\left(t z^{ \pm 1}\right) \prod_{1 \leq i<j \leq 4} \Gamma\left(t z_{i}^{2} z_{j}^{2} z^{-1}\right)}{\prod_{1 \leq i<j \leq 4} \Gamma\left(z_{i}^{ \pm 2} z_{j}^{ \pm 2}\right)} \\
\quad \times \frac{\prod_{j=1}^{4} \Gamma\left(t\left(z_{j}^{2} z^{-1}\right)^{ \pm 1}\right)}{\prod_{j=1}^{4} \Gamma\left(z_{j}^{ \pm 2}\right)} \prod_{i=1}^{N_{f}} \prod_{j=1}^{4} \Gamma\left(s_{i} z_{j}^{ \pm 2}\right) \prod_{j=1}^{4} \frac{d z_{j}}{2 \pi \mathrm{i} z_{j}}
\end{gathered}
$$


where $z=z_{1} z_{2} z_{3} z_{4}$ and the balancing condition reads $s t^{2}=(p q)^{\frac{1}{2}\left(N_{f}-5\right)}$ with $s=\prod_{i=1}^{N_{f}} s_{i}$. In the magnetic theory we have (with $\prod_{j=1}^{N_{f}-4} y_{j}=1$ )

$$
\begin{aligned}
& I_{M}=\Gamma\left(t^{2}\right) \prod_{i=1}^{N_{f}} \Gamma\left(s_{i}^{2}, t^{2} s_{i}\right) \prod_{1 \leq i<j \leq N_{f}} \Gamma\left(s_{i} s_{j}\right) \frac{(p ; p)_{\infty}^{N_{f}-5}(q ; q)_{\infty}^{N_{f}-5}}{\left(N_{f}-4\right) !} \\
& \times \int_{\mathbb{T}^{N_{f}-5}} \prod_{1 \leq i<j \leq N_{f}-4} \frac{\Gamma\left((p q)^{\frac{1}{N_{f}-4}} y_{i}^{-1} y_{j}^{-1}\right)}{\Gamma\left(y_{i} y_{j}^{-1}, y_{i}^{-1} y_{j}\right)} \prod_{i=1}^{N_{f}} \prod_{j=1}^{N_{f}-4} \Gamma\left((p q)^{\frac{N_{f}-5}{2\left(N_{f}-4\right)}} s_{i}^{-1} y_{j}\right) \\
& \quad \times \prod_{j=1}^{N_{f}-4} \Gamma\left((p q)^{\frac{1}{N_{f}-4}} y_{j}^{-2},(p q)^{\frac{N_{f}-3}{2\left(N_{f}-4\right)}} t^{-2} y_{j}^{-1},(p q)^{\frac{N_{f}-5}{2\left(N_{f}-4\right)}} y_{j}\right) \prod_{j=1}^{N_{f}-5} \frac{d y_{j}}{2 \pi \mathrm{i} y_{j}}
\end{aligned}
$$

5.8. $G=S O(10)$ and $F=S U\left(N_{f}\right) \times U(1)$. The electric theory is [95]

\begin{tabular}{|c|c|c|c|c|}
\hline & $S O(10)$ & $S U\left(N_{f}\right)$ & $U(1)$ & $U(1)_{R}$ \\
\hline$Q$ & $f$ & $f$ & -1 & $1-\frac{8}{N_{f}+2}$ \\
$P$ & $s$ & 1 & $\frac{N_{f}}{2}$ & $1-\frac{8}{N_{f}+2}$ \\
\hline
\end{tabular}

while the magnetic theory is

\begin{tabular}{|c|c|c|c|c|}
\hline & $S U\left(N_{f}-5\right)$ & $S U\left(N_{f}\right)$ & $U(1)$ & $U(1)_{R}$ \\
\hline$w$ & $T_{S}$ & 1 & 0 & $\frac{2}{N_{f}-5}$ \\
$q$ & $\bar{f}$ & $\bar{f}$ & 1 & $\frac{8}{N_{f}+2}-\frac{1}{N_{f}-5}$ \\
$q^{\prime}$ & $f$ & 1 & $-N_{f}$ & $-1+\frac{16}{N_{f}+2}+\frac{1}{N_{f}-5}$ \\
$M$ & 1 & $T_{S}$ & -2 & $2-\frac{16}{N_{f}+2}$ \\
$Y$ & 1 & $f$ & $N_{f}-1$ & $3-\frac{24}{N_{f}+2}$ \\
\hline
\end{tabular}

where $7 \leq N_{f} \leq 21$. The SCIs are

$$
\begin{aligned}
I_{E}=\frac{(p ; p)_{\infty}^{5}(q ; q)_{\infty}^{5}}{2^{4} 5 !} & \int_{\mathbb{T}^{5}} \frac{\Gamma(t Z) \prod_{j=1}^{5} \Gamma\left(t z_{j}^{2} Z^{-1}\right) \prod_{1 \leq i<j \leq 5} \Gamma\left(t Z z_{i}^{-2} z_{j}^{-2}\right)}{\prod_{1 \leq i<j \leq 5} \Gamma\left(z_{i}^{ \pm 2} z_{j}^{ \pm 2}\right)} \\
& \times \prod_{i=1}^{N_{f}} \prod_{j=1}^{5} \Gamma\left(s_{i} z_{j}^{ \pm 2}\right) \prod_{j=1}^{5} \frac{d z_{j}}{2 \pi \mathrm{i} z_{j}}
\end{aligned}
$$

where $s t^{2}=(p q)^{\frac{N_{f}}{2}-3}, s=\prod_{i=1}^{N_{f}} s_{i}, Z=z_{1} z_{2} z_{3} z_{4} z_{5}$, and $\left(\right.$ with $\left.\prod_{j=1}^{N_{f}-5} y_{j}=1\right)$

$$
\begin{gathered}
I_{M}=\prod_{1 \leq i<j \leq N_{f}} \Gamma\left(s_{i} s_{j}\right) \prod_{i=1}^{N_{f}} \Gamma\left(s_{i}^{2}, t^{2} s_{i}\right) \frac{(p ; p)_{\infty}^{N_{f}-6}(q ; q)_{\infty}^{N_{f}-6}}{\left(N_{f}-5\right) !} \\
\times \int_{\mathbb{T}^{N_{f}-6}} \prod_{1 \leq i<j \leq N_{f}-5} \frac{\Gamma\left((p q)^{\frac{1}{N_{f}-5}} y_{i} y_{j}\right)}{\Gamma\left(y_{i}^{-1} y_{j}, y_{i} y_{j}^{-1}\right)} \prod_{i=1}^{N_{f}-5} \Gamma\left((p q)^{\frac{1}{N_{f}-5}} y_{i}^{2},(p q)^{\frac{N_{f}-4}{2\left(N_{f}-5\right)}} t^{-2} y_{i}\right) \\
\times \prod_{i=1}^{N_{f}} \prod_{j=1}^{N_{f}-5} \Gamma\left((p q)^{\frac{N_{f}-6}{2\left(N_{f}-5\right)}} s_{i}^{-1} y_{j}^{-1}\right) \prod_{j=1}^{N_{f}-6} \frac{d y_{j}}{2 \pi \mathrm{i} y_{j}} .
\end{gathered}
$$

An interesting fact is that fixing $s_{1}=1$ and $t=\sqrt{p q}$ in both integrals, we come to SCIs of the original Seiberg duality between $S O(9)$ and $S O\left(N_{f}-5\right)$ gauge theories with $N_{f}$ quarks 
in the fundamental representation [104]. A connection between these dualities was understood first from the physical point of view in 95, and our observation is that SCIs are connected as well after imposing appropriate constraints. The residue calculus similar to that of [25] should be applied to the electric theory. In the limit $s_{1} \rightarrow 1$ the integration contour is pinched by the poles coming from the term $\prod_{j=1}^{5} \Gamma\left(s_{1} z_{j}^{ \pm 2}\right)$. Picking up residues of the poles at $z_{j}=s_{1}^{ \pm 1 / 2}$ we come to SCI of $\mathcal{N}=1 \mathrm{SYM}$ theory with $S O(9)$ gauge group and $N_{f}$ quarks in the fundamental representation. In the magnetic SCI we have the multiplier $\Gamma\left(t^{2} s_{1}\right)$ vanishing in the discussed limit and further steps are a little tricky. For $N_{f}>5$ and $N_{f}$ odd it is convenient first to rescale $y_{i} \rightarrow(p q)^{-1 / 2\left(N_{f}-4\right)} y_{i}, i=1, \ldots, N_{f}-5$. Then the first residue comes from the pole at $y_{j}=\sqrt{p q}$, and other residues come from the poles $y_{2 i+1}=y_{2 i}, i=1, \ldots,\left(N_{f}-5\right) / 2$. Accurately computing all these sequential residues one can verify that the resulting integral describes SCI of the magnetic theory with $S O\left(N_{f}-5\right)$ gauge group having $N_{f}$ quarks in the fundamental representation and the gauge singlet baryon field in the $T_{S}$-representation of the flavor group $S U\left(N_{f}\right)$.

There is another nice reduction of dual theories observed in [95]. If we take $N_{f}=8$ then we can obtain $S$-duality for $\mathcal{N}=2$ SYM theory with $S U(2)$ gauge group and 4 hypermultiplets studied in detail in [106]. From the mathematical point of view we need to apply the following constraints in (5.17) and (5.18)

$$
s_{1} s_{5}=1, \quad s_{2} s_{6}=1, \quad s_{3} s_{7}=1, \quad s_{4}=1
$$

and then compute the residues of poles $z_{1}=s_{1}, z_{2}=s_{2}, z_{3}=s_{3}, z_{4}=s_{4}$ (and all their permutations) which leads to the equality of reduced SCIs

$$
\begin{aligned}
I_{E}^{\prime}= & \frac{(p ; p)_{\infty}(q ; q)_{\infty}}{2} \int_{\mathbb{T}} \frac{\Gamma\left(s_{8} z^{ \pm 2}, \sqrt{p q} s_{8}^{-\frac{1}{2}}\left(s_{1} s_{2} s_{3}\right)^{ \pm \frac{1}{2}} z^{ \pm 1}\right)}{\Gamma\left(z^{ \pm 2}\right)} \\
& \left.\times \prod_{i=1}^{3} \Gamma\left(\sqrt{p q} s_{8}^{-\frac{1}{2}}\left(s_{i}\left(s_{1} s_{2} s_{3}\right)^{-\frac{1}{2}}\right)\right)^{ \pm 1} z^{ \pm 1}\right) \frac{d z}{2 \pi \mathrm{i} z}
\end{aligned}
$$

and

$$
I_{M}^{\prime}=\frac{(p ; p)_{\infty}(q ; q)_{\infty}}{2} \int_{\mathbb{T}} \frac{\Gamma\left(s_{8} z^{ \pm 2}\right) \Gamma^{2}\left(\sqrt{p q} s_{8}^{-\frac{1}{2}} z^{ \pm 1}\right)}{\Gamma\left(z^{ \pm 2}\right)} \prod_{i=1}^{3} \Gamma\left(\sqrt{p q} s_{8}^{-\frac{1}{2}} s_{i}^{ \pm 1} z^{ \pm 1}\right) \frac{d z}{2 \pi \mathrm{i} z}
$$

The equality $I_{E}^{\prime}=I_{M}^{\prime}$ is a particular case of the identity obtained in [16] with

$$
b=s_{8}, \quad t_{4}=\sqrt{p q} s_{8}^{-\frac{1}{2}}, \quad t_{i}=\sqrt{p q} s_{8}^{-\frac{1}{2}} s_{i}, i=1,2,3 .
$$

One can reduce also the duality considered in this section to the dualities studied in [33]. If we give vacuum expectation values to $k$ fundamental quarks in the electric theory, it breaks the gauge group to $S O(10-k)$ while in the magnetic side the gauge group remains the same [33], see Sect. 5.1 for a particular example when $k=5$. But these dualities should be considered with a care since, as we have shown in Sect. 5.1, instead of $S U(4)$ flavor symmetry group one has $S O(4)$ symmetry group. From SCIs point of view we should restrict some of the parameters to form the divergency $\propto \Gamma(1)$ in (5.18). Appearance of such a term in the magnetic index requires the residue calculus on the electric side. For example, the model considered in Sect. 5.1 is obtained from (5.17) and (5.18) by taking in these expressions the following limits

$$
s_{N_{f}-4} s_{N_{f}-3}=s_{N_{f}-2} s_{N_{f}-1}=s_{N_{f}}=1 \text {, }
$$

with the subsequent replacement $N_{f} \rightarrow N_{f}+5$ and identification $s_{N_{f}-2}=u_{1}, s_{N_{f}-4}=u_{2}$. 
A more general duality was proposed in [11, 73] having on the electric side the same $S O(10)$ gauge group with $N_{f}$ vectors and $N_{k}$ spinors. The magnetic dual side was conjectured to be a quiver gauge theory with $S U$ and $S P$ gauge groups. Again, as in Sect. 5.1, we have not found evidence for this duality from SCIs technique point of view, except of the obvious cases when the dual gauge group contains only one simple component or when the theory $s$-confines, in which cases one obtains known dualities. Anyway, we are not considering quiver gauge theories in this work, so we leave open the detailed analysis of repairing the general duality of [11, 73]. As in Sect. 5.1, perhaps this question may be settled by a reduction from an even more general unknown duality for $S O(N), N>10$, gauge group.

\section{Matrix models AND AN Elliptic Deformation of $2 d$ CFT}

Main inspiration for this section comes from paper [102], where a $q$-deformed $2 d$ CFT and the corresponding matrix model description in terms of the Jackson integrals was proposed. From EHIs' point of view there is a natural way to propose a generalization of CFT to the elliptic and different $q$-deformed levels. $q$-Extensions of the Virasoro algebra have been considered already some time ago [5, 42, 80] (see also [4, 6, 43] for a recent discussion). Here we propose expressions for the three- and four-point correlation functions presumably associated with new hypothetical $q$-deformations and an elliptic deformation of $2 d$ CFT. For that we employ various known generalizations of the Selberg integral, the basic integral appearing in calculations of the three-point correlation function in $2 d$ CFT.

6.1. Elliptic Selberg integral. The following elliptic generalization of the Selberg integral attached to the root system $B C_{N}$ was discovered in [25, 26]:

$$
\begin{gathered}
\frac{(p ; p)_{\infty}^{N}(q ; q)_{\infty}^{N}}{2^{N} N !} \int_{\mathbb{T}^{N}} \prod_{1 \leq i<j \leq N} \frac{\Gamma\left(t z_{i}^{ \pm 1} z_{j}^{ \pm 1}\right)}{\Gamma\left(z_{i}^{ \pm 1} z_{j}^{ \pm 1}\right)} \prod_{j=1}^{N} \frac{\prod_{i=1}^{6} \Gamma\left(t_{i} z_{j}^{ \pm 1}\right)}{\Gamma\left(z_{j}^{ \pm 2}\right)} \prod_{j=1}^{N} \frac{d z_{j}}{2 \pi \mathrm{i} z_{j}} \\
=\prod_{j=1}^{N}\left(\frac{\Gamma\left(t^{j}\right)}{\Gamma(t)} \prod_{1 \leq i<k \leq 6} \Gamma\left(t^{j-1} t_{i} t_{k}\right)\right),
\end{gathered}
$$

where $|t|,\left|t_{j}\right|<1$ and the balancing condition reads $t^{2(N-1)} \prod_{i=1}^{6} t_{i}=p q$. This integral describes $\mathcal{N}=1 s$-confining SYM theory with $S P(2 N)$ gauge group, one chiral superfield in the $T_{A^{-}}$ representation of $S P(2 N)$, and 6 quarks [117]. This physical application provides a matrix model interpretation of formula (6.1). Note also that this integral describes the normalization of a particular eigenstate of a relativistic Calogero-Sutherland type model [113].

We postulate that the chiral part of the three-point correlation function of a hypothetical elliptic deformation of $2 d$ CFT based on an elliptic extension of the Virasoro algebra is given by integral (6.1) admitting exact evaluation. This proposition fits the fact that in all known variations of $2 d$ CFT the three-point function is computable exactly. Note that in [43] a simple elliptic deformation of the free bosonic field algebra was proposed, but its relevance to our construction is not clear, in particular, the number and meaning of the parameters $t_{j}$ are not evident in this case.

6.2. $q$-Selberg integral. Different reductions of EHIs were systematically investigated in [98] (see also [17]). First we reduce integral (6.1) to the trigonometric level and then to the standard Selberg integral. The limit $p \rightarrow 0$ is not straightforward due to the balancing condition which 
we get rid of by substituting in (6.1) $t_{6}=p q /\left(t^{2(N-1)} T\right)$, where $T=\prod_{i=1}^{5} t_{i}$, and obtain

$$
\begin{aligned}
& \frac{(p ; p)_{\infty}^{N}(q ; q)_{\infty}^{N}}{2^{N} N !} \int_{\mathbb{T}^{N}} \prod_{1 \leq i<j \leq N} \frac{\Gamma\left(t z_{i}^{ \pm 1} z_{j}^{ \pm 1}\right)}{\Gamma\left(z_{i}^{ \pm 1} z_{j}^{ \pm 1}\right)} \prod_{j=1}^{N} \frac{\prod_{i=1}^{5} \Gamma\left(t_{i} z_{j}^{ \pm 1}\right)}{\Gamma\left(t^{2(N-1)} T z_{j}^{ \pm 1}\right) \Gamma\left(z_{j}^{ \pm 2}\right)} \prod_{j=1}^{N} \frac{d z_{j}}{2 \pi \mathrm{i} z_{j}} \\
& =\prod_{j=1}^{N}\left(\frac{\Gamma\left(t^{j}\right)}{\Gamma(t)} \prod_{1 \leq i<k \leq 5} \Gamma\left(t^{j-1} t_{i} t_{k}\right) \prod_{i=1}^{5} \frac{1}{\Gamma\left(t^{N+j-2} T / t_{i}\right)}\right) .
\end{aligned}
$$

After setting $p=0$ with fixed $t_{i}$ and subsequently $t_{5}=0$ one obtains the trigonometric $q$-Selberg integral of Gustafson [55]

$$
\begin{aligned}
& \frac{1}{2^{N} N !} \int_{\mathbb{T}^{N}} \prod_{1 \leq i<j \leq N} \frac{\left(z_{i}^{ \pm 1} z_{j}^{ \pm 1} ; q\right)_{\infty}}{\left(t z_{i}^{ \pm 1} z_{j}^{ \pm 1} ; q\right)_{\infty}} \prod_{j=1}^{N} \frac{\left(z_{j}^{ \pm 2} ; q\right)_{\infty}}{\prod_{i=1}^{4}\left(t_{i} z_{j}^{ \pm 1} ; q\right)_{\infty}} \prod_{j=1}^{N} \frac{d z_{j}}{2 \pi \mathrm{i} z_{j}} \\
& \quad=\prod_{j=1}^{N}\left(\frac{(t ; q)_{\infty}}{\left(t^{j} ; q\right)_{\infty}(q ; q)_{\infty}}\left(t^{N+j-2} t_{1} t_{2} t_{3} t_{4} ; q\right)_{\infty} \prod_{1 \leq i<k \leq 4} \frac{1}{\left(t^{j-1} t_{i} t_{k} ; q\right)_{\infty}}\right) .
\end{aligned}
$$

Again, as above, we postulate that the three-point correlation function of a hypothetical $2 d$ CFT based on a (yet unknown) q-deformed Virasoro algebra is given by function (6.3). Note that it is described by the standard contour integral and not the Jackson $q$-integral, as suggested in [102].

6.3. Reduction to the Selberg integral. To obtain the Selberg integral one should carefully take the limit $q \rightarrow 1^{-}$. To simplify the left-hand side of formula (6.3) we use the relation

$$
\lim _{q \rightarrow 1^{-}} \frac{\left(q^{a} z ; q\right)_{\infty}}{(z ; q)_{\infty}}=(1-z)^{-a}
$$

and the duplication formula $\left(z^{2} ; q\right)_{\infty}=\left( \pm z, \pm q^{\frac{1}{2}} z ; q\right)_{\infty}$. To simplify the right-hand side expression we replace infinite products by the Jackson $q$-gamma function

$$
\Gamma_{q}(x)=\frac{(q ; q)_{\infty}}{\left(q^{x} ; q\right)_{\infty}}(1-q)^{1-x}, \quad \Gamma_{q}(x) \underset{q \rightarrow 1}{=} \Gamma_{r a t}(x)
$$

where $\Gamma_{\text {rat }}(x)$ is the Euler gamma function. Now we denote the parameters entering (6.3) as

$$
t=q^{\gamma}, \quad t_{1}=q^{\alpha-\frac{1}{2}}, \quad t_{2}=-q^{\beta-\frac{1}{2}}, \quad t_{3}=q^{\frac{1}{2}}, \quad t_{4}=-q^{\frac{1}{2}} .
$$

On the left-hand side of (6.3) we change also the integration variables $z_{j}=e^{i \theta_{j}}$ and denote $x_{i}=\left(1+\cos \theta_{i}\right) / 2$. Finally, for fixed $\alpha, \beta, \gamma$, we can take safely the limit $q \rightarrow 1^{-}$, which brings us to the standard Selberg integral [3]

$$
\begin{aligned}
\int_{0}^{1} & \ldots \int_{0}^{1} \prod_{j=1}^{N} x_{j}^{\alpha-1}\left(1-x_{j}\right)^{\beta-1} \prod_{1 \leq i<j \leq N}\left|x_{i}-x_{j}\right|^{2 \gamma} d x_{1} \ldots d x_{N} \\
& =\prod_{j=1}^{N} \frac{\Gamma_{r a t}(\alpha+(j-1) \gamma) \Gamma_{r a t}(\beta+(j-1) \gamma) \Gamma_{r a t}(1+j \gamma)}{\Gamma_{r a t}(\alpha+\beta+(n+j-2) \gamma) \Gamma_{r a t}(1+\gamma)},
\end{aligned}
$$

where the integral converges for $\Re \alpha, \Re \beta>0, \Re \gamma>-\min (1 / N, \Re \alpha /(N-1), \Re \beta /(N-1))$. Expression (6.7) defines the $\beta$-deformed matrix integral and gives the three-point function of the standard undeformed $2 d$ CFT, see, e.g., Sect. 4.1 of [102]. 
6.4. A higher order elliptic Selberg integral. A two parameter extension of the elliptic Selberg integral (6.1) is given by the integral

$$
V\left(t_{1}, \ldots, t_{8} ; t ; p, q\right)=\frac{(p ; p)_{\infty}^{N}(q ; q)_{\infty}^{N}}{2^{N} N !} \int_{\mathbb{T}^{N}} \prod_{1 \leq i<j \leq N} \frac{\Gamma\left(t z_{i}^{ \pm 1} z_{j}^{ \pm 1}\right)}{\Gamma\left(z_{i}^{ \pm 1} z_{j}^{ \pm 1}\right)} \prod_{j=1}^{N} \frac{\prod_{i=1}^{8} \Gamma\left(t_{i} z_{j}^{ \pm 1}\right)}{\Gamma\left(z_{j}^{ \pm 2}\right)} \prod_{j=1}^{N} \frac{d z_{j}}{2 \pi \mathrm{i} z_{j}}
$$

where the balancing condition reads $t^{2(N-1)} \prod_{i=1}^{8} t_{i}=(p q)^{2}$. The symmetry transformation properties of this integral were found in [111] for $N=1$ and in [97] for general $N$. We are not presenting them here explicitly for brevity (for $N=1$ they are described by formula (2.19)). We conjecture that integral (6.8) coincides with the four-point correlation function for an elliptic deformation of $2 d$ CFT for which the elliptic Selberg integral defines the three-point function. Then the $s$ - $t$-channels duality for this four-point function is described by known symmetries of (6.8).

Again, taking appropriately the (trigonometric) limit $p \rightarrow 0$ we can come to the two parameter extension of the $q$-Selberg integral with further degeneration to the rational level [98]. For arbitrary $N$ and a special choice of one of the parameters, there emerges the ${ }_{2} F_{1}$-hypergeometric function describing the chiral part of the four point correlation function (see formula (4.9) in [102]). General ${ }_{2} F_{1}$-function is obtained also for $N=1$, we skip explicit description of these well known results. In [80], the four point correlation function of a $q$-deformed CFT was connected to a $q$-analog of the ${ }_{2} F_{1}$-hypergeometric function. We conjecture that an appropriate elliptic analog of the latter correlation function will be expressed in terms of the $V$-function of [114] given by $N=1$ case of (6.8). Apart from the mentioned limit $p \rightarrow 0$, there exists a different degenerating limit for the elliptic Selberg integral to the hyperbolic $q$-hypergeometric level [27], which was discussed recently in detail in [37] where one of the resulting integrals was interpreted as the partition function of a particular $3 d \mathcal{N}=2$ supersymmetric field theory model (it is also expected to play a proper role in $2 d$ CFT deformations).

\section{Connection to the knot theory}

In this section we discuss the connection of partition functions for some $3 d$ supersymmetric field theories and non-supersymmetric CS theories with the complexified gauge groups to topological invariants of the knot theory [28, 30, 32, 58, 59]. In [37], the theory of hyperbolic $q$-hypergeometric integrals has been exploited for checking and searching for $3 d$ supersymmetric dualities. Earlier it was proposed in [58] that the state integrals for knots are also defined in terms of such integrals. In an independent approach to state integrals [28], Dimofte proposed a new expression for the figure-eight knot state integral and conjectured that it coincides with the one of [58]. Using the approach of [37] we prove here this conjecture, as well as some other similar identities needed in [29].

The hyperbolic $q$-hypergeometric integrals can be rigorously obtained as reductions of the EHIs [98] (for an earlier formal approach see, e.g., [27], and for a detailed explicit analysis of reducing many integrals see [15]). The reduction procedure inherits certain pieces of the unique symmetry properties of the original integrals and yields many nontrivial identities at the hyperbolic level. The resulting hyperbolic integrals and identities emerge in various physical problems. Here we stress that they describe partition functions for $3 d$ supersymmetric theories living on the squashed three-sphere and the state integrals for the knots. As the most recent example of their relevance, we mention a generalization of the AGT duality [2] to the duality inspired by the $(3+3)$-dimensional theories [30, 38, 61], with the non-supersymmetric CS theory 
living on a $3 d$ manifold $\mathcal{M}$ on the one side and $3 d \mathcal{N}=2$ supersymmetric theory living on the squashed sphere on the other side.

7.1. The figure-eight knot. We start from the notation for hyperbolic gamma function used in 37, 115. This function appeared in [40] under the name "noncompact quantum dilogarithm". For $q=e^{2 \pi \mathrm{i} \omega_{1} / \omega_{2}}$ and $\tilde{q}=e^{-2 \pi \mathrm{i} \omega_{2} / \omega_{1}}$ with $|q|<1$ we define

$$
\gamma\left(u ; \omega_{1}, \omega_{2}\right)=\frac{\left(e^{2 \pi \mathrm{i} u / \omega_{1}} \tilde{q} ; \tilde{q}\right)_{\infty}}{\left(e^{2 \pi \mathrm{i} u / \omega_{2}} ; q\right)_{\infty}}, \quad \gamma^{(2)}\left(u ; \omega_{1}, \omega_{2}\right)=e^{-\pi \mathrm{i} B_{2,2}(u) / 2} \gamma\left(u ; \omega_{1}, \omega_{2}\right),
$$

where $B_{2,2}\left(u ; \omega_{1}, \omega_{2}\right)$ is the second order Bernoulli polynomial,

$$
B_{2,2}\left(u ; \omega_{1}, \omega_{2}\right)=\frac{u^{2}}{\omega_{1} \omega_{2}}-\frac{u}{\omega_{1}}-\frac{u}{\omega_{2}}+\frac{\omega_{1}}{6 \omega_{2}}+\frac{\omega_{2}}{6 \omega_{1}}+\frac{1}{2} .
$$

For $\operatorname{Re}\left(\omega_{1}\right), \operatorname{Re}\left(\omega_{2}\right)>0$ and $0<\operatorname{Re}(u)<\operatorname{Re}\left(\omega_{1}+\omega_{2}\right)$ one has the following integral representation for the hyperbolic gamma function

$$
\gamma^{(2)}\left(u ; \omega_{1}, \omega_{2}\right)=\exp \left(-\mathrm{PV} \int_{\mathbb{R}} \frac{e^{u x}}{\left(1-e^{\omega_{1} x}\right)\left(1-e^{\omega_{2} x}\right)} \frac{d x}{x}\right),
$$

where 'PV' means the principal value integral.

Different notations and names for slight modifications of this function are used in the literature, most of them were explicitly described in Appendix A of [115]. In [32], the following "quantum dilogarithm" is employed

$$
\Phi(z ; \tau)=\frac{(-e(z+\tau / 2) ; e(\tau))_{\infty}}{(-e((z-1 / 2) / \tau) ; e(-1 / \tau))_{\infty}},
$$

where $e(x)=e^{2 \pi i x}$. One can easily find by comparison that

$$
\Phi(z ; \tau)=\gamma\left(\frac{\omega_{1}+\omega_{2}}{2}+z \omega_{2} ; \omega_{1}, \omega_{2}\right)^{-1}, \quad \tau=\frac{\omega_{1}}{\omega_{2}} .
$$

Consider the so-called state integral for the figure eight knot $\mathbf{4}_{\mathbf{1}}$ which was found first by Hikami in [58] and studied further in [59, 32, 28, 30]. We stick to the notation of paper [32] where this integral is given by formula (4.46) and has the form

$$
I=\frac{e^{2 \pi \mathrm{i} u / \hbar+u}}{\sqrt{2 \pi \hbar}} \int_{-\infty}^{\infty} \frac{\Phi((p-u) / 2 \pi \mathrm{i} ; \hbar / \pi \mathrm{i})}{\Phi(-(p+u) / 2 \pi \mathrm{i} ; \hbar / \pi \mathrm{i})} e^{-2 p u / \hbar} d p .
$$

This integral describes also the partition function of non-supersymmetric CS theory with the complexified gauge group $S L(2, \mathbb{C})$ living on the $3 d$ manifold $\mathcal{M}=S^{3} \backslash \mathbf{4}_{\mathbf{1}}$ [32. Denoting $\omega_{1}=b, \omega_{2}=b^{-1}, \tau=b^{2}$ and changing the variables $p \rightarrow 2 \pi \mathrm{i} p, u \rightarrow 2 \pi \mathrm{i} u, \hbar \rightarrow \pi \mathrm{i} \tau$ in (7.3), we obtain

$$
I=e^{2 \pi \mathrm{i}\left(2+b^{2}\right) u / b^{2}} \int_{-\mathrm{i} \infty}^{\mathrm{i} \infty} \frac{\Phi\left(p-u ; b^{2}\right)}{\Phi\left(-p-u ; b^{2}\right)} e^{-8 \pi \mathrm{i} p u / b^{2}} d p,
$$

where we drop the multiplier $\sqrt{2 \pi} / \mathrm{i} \sqrt{\hbar}$ in front of the integral. Using relation (7.2), we can write

$$
I=e^{2 \pi \mathrm{i}\left(2+b^{2}\right) u / b^{2}} \int_{-\mathrm{i} \infty}^{\mathrm{i} \infty} \frac{\gamma\left(\frac{b+1 / b}{2}-\frac{p+u}{b} ; b, b^{-1}\right)}{\gamma\left(\frac{b+1 / b}{2}+\frac{p-u}{b} ; b, b^{-1}\right)} e^{-8 \pi \mathrm{i} p u / b^{2}} d p .
$$


We apply the inversion formula $\gamma\left(u, b+1 / b-u ; b, b^{-1}\right)=e^{\pi \mathrm{i} B_{2,2}\left(u ; b, b^{-1}\right)}$ to move the denominator $\gamma$-function to the numerator and pass from the $\gamma$-function to the $\gamma^{(2)}$-function. This yields another form of the integral:

$$
I=e^{2 \pi \mathrm{i}\left(2+b^{2}\right) u / b^{2}} \int_{-\mathrm{i} \infty}^{\mathrm{i} \infty} \gamma^{(2)}\left(\frac{b+1 / b}{2}-\frac{p \pm u}{b} ; b, b^{-1}\right) e^{-6 \pi \mathrm{i} p u / b^{2}} d p .
$$

Consider now integral (6.77) from [28] (in the suggested there normalization without the multiplier $\left.2^{-1 / 2} e^{\left(4 \pi^{2}-\hbar^{2}\right) / 24 \hbar^{2}}\right)$. After changing the notation in it similar to the integral $I$, we come to the following expression

$$
\widetilde{I}=e^{-2 \pi \mathrm{i} u} \int_{-\mathrm{i} \infty}^{\mathrm{i} \infty} \gamma^{(2)}\left(\frac{b+1 / b}{2}-\frac{p \pm u}{b} ; b, b^{-1}\right) e^{6 \pi \mathrm{i} p u / b^{2}} d p .
$$

One can see that the difference between expressions (17.6) and (7.7) is in the coefficients in front of the integrals and in the sign of the exponent of the integrand.

Let us take now the $n=1$ case of the integral $I I_{n,(3,3) * a}^{1}(\mu ;-; \lambda ; \tau)$ defined on page 218 of [15. Replacing the integration variable $x \rightarrow p / b$ in it and changing slightly its normalizing multiplier, we come to the integral

$$
Z_{E}\left(\mu_{1}, \mu_{2}, \sigma\right)=\int_{-\mathrm{i} \infty}^{\mathrm{i} \infty} \prod_{i=1}^{2} \gamma^{(2)}\left(\mu_{i}-p / b ; b, b^{-1}\right) e^{\pi \mathrm{i} \sigma p / b} d p
$$

where $\mu_{1}, \mu_{2}$, and $\sigma$ are some free parameters.

Remarkably, our original integral of interest $I$ (7.6) is a special subcase of (7.8), which is obtained after imposing the constraints

$$
\mu_{1}=(b+1 / b) / 2-u / b, \quad \mu_{2}=(b+1 / b) / 2+u / b, \quad \sigma=-6 u / b .
$$

Using the results of [37], we see that expression (7.7) with arbitrary $\mu_{1}, \mu_{2}, \sigma$ describes the partition function (that is why it is denoted as $Z_{E}$ ) of $3 d \mathcal{N}=2$ theory living on the squashed three-sphere with the $U(1)$ gauge group and two quarks, which is referred to as the "electric theory". The global symmetry group is $S U(2) \times U(1)_{A} \times U(1)_{R}$. We do not discuss the vector superfield having well known properties. The matter content with the corresponding charges is presented in the following table

\begin{tabular}{|c|c|c|c|c|}
\hline & $U(1)$ & $S U(2)$ & $U(1)_{A}$ & $U(1)_{R}$ \\
\hline$q$ & -1 & $f$ & 1 & $\frac{1}{2}$ \\
\hline
\end{tabular}

Integral (7.8) has the transformation formula described in Theorem 5.6.20 of [15]:

$$
e^{\pi \mathrm{i}\left(4 \mu_{1} \mu_{2}-\mu_{3}^{2}+(b+1 / b) \mu_{3}-(b+1 / b)^{2} / 4\right) / 2+\pi \mathrm{i}\left(b^{2}+1 / b^{2}\right) / 24} Z_{E}\left(\mu_{1}, \mu_{2}, 2 \mu_{3}-\mu_{1}-\mu_{2}\right)=Z_{M}(\underline{\mu} ; \lambda),
$$

where

$$
Z_{M}\left(\mu_{1}, \mu_{2}, \mu_{3} ; \lambda\right)=\int_{-\mathrm{i} \infty}^{\mathrm{i} \infty} \prod_{i=1}^{3} \gamma^{(2)}\left(\mu_{i}-p / b ; b, b^{-1}\right) e^{\pi \mathrm{i} \lambda p / b-3 \pi \mathrm{i} p^{2} / 2 b^{2}} d p
$$

with $\mu_{3}$ being a new parameter introduced through the balancing condition $\mu_{1}+\mu_{2}+\mu_{3}=$ $\lambda-(b+1 / b) / 2$. This condition relates fugacities associated with the $S U(3)$ flavor group acting on quarks and the Fayet-Illiopoulos term $\lambda$.

Expression (17.11) represents the partition function of a "magnetic theory" defined as the $3 d$ $\mathcal{N}=2$ CS theory with $U(1)_{3 / 2}$ gauge group and 3 quarks. The global symmetry group of the magnetic theory is $S U(3) \times U(1)_{A} \times U(1)_{R}$. Note that the flavor groups of the electric and magnetic theories differ although the number of independent variables is the same for both 
statistical sums. The matter fields together with the corresponding charges are presented in the table below

\begin{tabular}{|c|c|c|c|c|}
\hline & $U(1)_{3 / 2}$ & $S U(3)$ & $U(1)_{A}$ & $U(1)_{R}$ \\
\hline$q$ & -1 & $f$ & 1 & $\frac{1}{2}$ \\
\hline
\end{tabular}

The duality between these two $3 d$ theories is one of very many dualities not considered in [37] due to their abundance.

Now we can easily prove the equality of two forms of the figure-eight knot state integrals (7.6) and (17.7),$I=\widetilde{I}$. Evidently, expression (7.11) is symmetric in parameters $\mu_{1}, \mu_{2}$, and $\mu_{3}$. If we substitute in the left-hand side of (7.10) restrictions (7.9), we obtain the integral $I$ up to some factor. Now we permute the parameters in the left-hand side $\left(\mu_{1}, \mu_{2}, \mu_{3}\right) \rightarrow\left(\mu_{3}, \mu_{1}, \mu_{2}\right)$ (which is permitted because of the identity) and substitute anew the same restrictions (7.9). As a result we obtain the integral $\tilde{I}$ up to the same multiplier as before. Equating both expressions, we prove that $I=\tilde{I}$.

Moreover, we can use further this permutational symmetry and replace in the left-hand side of (7.10) $\left(\mu_{1}, \mu_{2}, \mu_{3}\right) \rightarrow\left(\mu_{2}, \mu_{3}, \mu_{1}\right)$, and impose constraints (7.9). As a result we come to one more form of the figure-eight knot state integral

$$
I=\tilde{I}=\widehat{I}:=e^{2 \pi \mathrm{i} u(1-6 u) / b^{2}} \int_{-\mathrm{i} \infty}^{\mathrm{i} \infty} \gamma^{(2)}\left(\frac{b+1 / b}{2}-\frac{3 u+p}{b}, \frac{b+1 / b}{2}+\frac{u-p}{b} ; b, b^{-1}\right) d p
$$

which was not considered in [28, 32, 58, 59].

As observed in [30, 38], there is an extension of the AGT duality [2] to the situation when the 6-dimensional space-time is descomposed as a $(3+3) d$ manifold with the duality relation between the complexified CS theories living on some $3 d$ manifold $\mathcal{M}$ and $3 d$ supersymmetric field theories. Our equality of partition functions (7.10) gives an explicit example of such a duality. In it the CS theory with $S L(2, \mathbb{C})$ gauge group on $\mathcal{M}=S^{3} \backslash \mathbf{4}_{\mathbf{1}}$ is dual to the $3 d$ theory with $U(1)$ gauge group and two flavors, which is also dual to the $3 d$ CS theory with $U(1)_{3 / 2}$ gauge group and three flavors, as described above.

Now we are coming to the main point of this section, namely, to derivation of the identities presented above from the theory of EHIs. Identity (17.10) arises from the reduction of a transformation formula of [11] for the elliptic extension of Euler-Gauss hypergeometric function (2.18). From the physical point of view EHIs describe SCIs for $4 d$ supersymmetric field theories and, analogously to [50], we can claim that important ingredients of the knot theory are coming from the $4 d$ space-time. In the considered example, the state integral model for the figure-eight knot is obtained from $4 d \mathcal{N}=1 \mathrm{SYM}$ theory with $S P(2)$ gauge group and 8 quarks, which was studied in detail in [116].

The $V$-function obeys symmetry transformation (2.19). First, we reduce it to the level of hyperbolic $q$-hypergeometric integrals by means of the reparametrization of variables

$$
y=e^{2 \pi \mathrm{i} r z}, \quad t_{j}=e^{2 \pi \mathrm{i} r \mu_{j}}, \quad j=1, \ldots, 8, \quad p=e^{2 \pi \mathrm{i} b r}, \quad q=e^{2 \pi \mathrm{i} r / b},
$$

(here the base parameter $p$ should not be mixed up with the integration variable $p$ in (7.3)) and the subsequent limit $r \rightarrow 0$. In this limit the elliptic gamma function has the asymptotics 101

$$
\Gamma\left(e^{2 \pi \mathrm{i} r z} ; e^{2 \pi \mathrm{i} r b}, e^{2 \pi \mathrm{i} r / b}\right) \underset{r \rightarrow 0}{=} e^{-\pi \mathrm{i}(2 z-b-1 / b) / 12 r} \gamma^{(2)}\left(z ; b, b^{-1}\right) .
$$

Using it in the reduction, one obtains an integral lying on the top of a list of integrals emerging as degenerations of the $V$-function (we omit some simple diverging exponential multiplier 
appearing in this limit),

$$
I_{h}\left(\mu_{1}, \ldots, \mu_{8}\right)=\int_{-\mathrm{i} \infty}^{\mathrm{i} \infty} \frac{\prod_{i=1}^{8} \gamma^{(2)}\left(\mu_{i} \pm z ; b, b^{-1}\right)}{\gamma^{(2)}\left( \pm 2 z ; b, b^{-1}\right)} d z
$$

with the balancing condition $\sum_{i=1}^{8} \mu_{i}=2(b+1 / b)$. It has the following symmetry transformation formula descending from the elliptic one

$$
I_{h}\left(\mu_{1}, \ldots, \mu_{8}\right)=\prod_{1 \leq i<j \leq 4} \gamma^{(2)}\left(\mu_{i}+\mu_{j}, \mu_{i+4}+\mu_{j+4} ; b, b^{-1}\right) I_{h}\left(\nu_{1}, \ldots, \nu_{8}\right),
$$

where $\nu_{i}=\mu_{i}+\xi, \nu_{i+4}=\mu_{i+4}-\xi, i=1,2,3,4$, and the parameter $\xi$ is

$$
2 \xi=\sum_{i=5}^{8} \mu_{i}-b-1 / b=b+1 / b-\sum_{i=1}^{4} \mu_{i}
$$

To get the desired transformation formula (7.10) one should use the following asymptotic formulas when some of the parameters go to infinity

$$
\begin{aligned}
\lim _{u \rightarrow \infty} e^{\frac{\pi \mathrm{i}}{2} B_{2,2}(u)} \gamma^{(2)}(u) & =1, \quad \text { for } \arg b<\arg u<\arg 1 / b+\pi, \\
\lim _{u \rightarrow \infty} e^{-\frac{\pi \mathrm{i}}{2} B_{2,2}(u)} \gamma^{(2)}(u) & =1, \quad \text { for } \arg b-\pi<\arg u<\arg 1 / b .
\end{aligned}
$$

The proof of formula (7.10) by van de Bult presented in [15] is rather bulky. Starting from the key transformation formula (17.16) one has to pass step by step from one level of complexity to another one in the list of integrals obtained from $I_{h}$ by diminishing the number of independent parameters. Therefore we are not presenting it here explicitly although it is very straightforward.

7.2. The trefoil knot. Let us apply the same procedure to the state integral model of the trefoil knot described by formula (6.59) in [28] (where we omit a coefficient in front of the integral):

$$
J=\int_{-\infty}^{\infty} \Phi\left(-\frac{p}{2 \pi \mathrm{i}} ; \frac{\hbar}{\pi \mathrm{i}}\right) \Phi\left(\frac{p-c}{2 \pi \mathrm{i}} ; \frac{\hbar}{\pi \mathrm{i}}\right) e^{p u / 2 \hbar} d p
$$

After rewriting this expression as in the figure-eight knot case (replacing $p \rightarrow 2 \pi \mathrm{i} p, c \rightarrow$ $2 \pi \mathrm{i} c, \hbar \rightarrow \pi \mathrm{i} \tau$, etc), we come to the integral

$$
J=e^{\pi \mathrm{i}\left(1+b^{4}-6 c^{2}\right) / 12 b^{2}} \int_{-\mathrm{i} \infty}^{\mathrm{i} \infty} \gamma^{(2)}\left(\frac{b+1 / b}{2}+\frac{p}{b}, \frac{b+1 / b}{2}-\frac{p-c}{b} ; b, b^{-1}\right) e^{\pi \mathrm{i} p(3 c-p) / b^{2}} d p .
$$

Consider now the integral $I I_{1,(3,3) a}^{1}(\mu, \nu ; \lambda)$ on page 218 in [15]. We choose the integration variable in it $z=p / b$, impose the constraints $\mu=(b+1 / b) / 2, \nu=(b+1 / b) / 2+c / b, \lambda=3 c / b$, and denote the resulting function as $\widetilde{Z}_{E}(\mu, \nu, \lambda)$ :

$$
\widetilde{Z}_{E}(\mu, \nu, \lambda)=\int_{-\mathrm{i} \infty}^{\mathrm{i} \infty} \gamma^{(2)}\left(\mu-z, \nu+z ; b, b^{-1}\right) e^{\pi \mathrm{i} \lambda z-\pi \mathrm{i} z^{2}} d z
$$

which describes the partition function of a $3 d \mathcal{N}=2$ SYM theory with $U(1)$ gauge group and two quarks. According to Theorem 5.6.19 of [15], it obeys the following transformation formula:

$$
\widetilde{Z}_{E}(\mu, \nu, \lambda)=\widetilde{Z}_{M}\left(\mu+\sigma^{\prime}, \nu-\sigma^{\prime}\right) e^{\pi \mathrm{i}\left(\lambda^{2}+(\mu+\nu)^{2}-2(b+1 / b)(\mu+\nu)\right) / 4},
$$


where $4 \sigma^{\prime}=\nu-\mu-\lambda$ and

$$
\widetilde{Z}_{M}(\alpha, \beta)=\frac{1}{2} \int_{-\mathrm{i} \infty}^{\mathrm{i} \infty} \frac{\gamma^{(2)}\left(\alpha \pm y, \beta \pm y ; b, b^{-1}\right)}{\gamma^{(2)}\left( \pm 2 y ; b, b^{-1}\right)} e^{-4 \pi \mathrm{i} y^{2}} d y
$$

which is the partition function of a $3 d \mathcal{N}=2 \mathrm{CS}$ theory with $S U(2)_{1 / 2}$ gauge group and two quarks. Comparing with [28, we see that integral (7.20) coincides with the product wavefunction in the transformed basis. To get the state integral model for the trefoil knot one has to specify $\mu+\nu=b+1 / b$. Then expression (7.18) simplifies (set $c=0$ in it and apply the inversion formula) becoming a Gaussian integral which is easily evaluated. Again, one can use equality (7.21) for the connection of $3 d$ complexified CS theory living on $\widetilde{\mathcal{M}}=S^{3} \backslash \mathbf{3}_{\mathbf{1}}$ with $3 d$ supersymmetric field theories.

7.3. Some other integrals. In the rest of this section we would like to consider some other hyperbolic integrals which appear in this context [18, 19, 41] and describe their connection to EHIs. There is nice Fourier transformation formula for the hyperbolic gamma function [18, 41] (in particular, in [28] it is given by formula (6.54)). Let us define

$$
J_{E}=\int_{-\mathrm{i} \infty}^{\mathrm{i} \infty} \gamma^{(2)}\left(\mu-z / b ; b, b^{-1}\right) e^{\pi \mathrm{i}\left(2 \lambda z / b-z^{2} / b^{2}\right) / 2} d z .
$$

To match the definition of [28] one should fix the parameters as $\mu=(b+1 / b) / 2, \lambda=2 x$. Expression (7.23) can be found in [15], where it is defined as integral $I I_{1,(3,2) a}^{0}(\mu ; \lambda)$. This integral is computable exactly, as described in Theorem 5.6.8 of [15],

$$
\begin{aligned}
J_{E}=J_{M} & :=\gamma^{(2)}\left((b+1 / b) / 4+\lambda / 2-\mu / 2 ; b, b^{-1}\right) \\
& \times e^{\pi \mathrm{i}\left(-3 \mu^{2}+(\lambda-(b+1 / b) / 2)^{2}+2 \mu(3 \lambda+(b+1 / b) / 2)\right) / 4-\pi \mathrm{i}\left(b^{2}+1 / b^{2}\right) / 24} .
\end{aligned}
$$

To see the coincidence with formula (6.54) from [28] one should take into account the inversion formula for the hyperbolic gamma functions. Physically, the equality $J_{E}=J_{M}$ is obtained from the reduction of SCIs for $4 d \mathcal{N}=1 \mathrm{SYM}$ theory with $S U(2)$ gauge group and 6 quarks and its dual, and, mathematically, it emerges as a reduction of the elliptic beta integral [110].

The equality $J_{E}=J_{M}$ defines one of the simplest examples of dualities between two $3 d$ supersymmetric field theories. The electric theory is a $3 d \mathcal{N}=2$ CS theory with $U(1)_{1 / 2}$ gauge group and one quark $Q$, while the magnetic theory is just a free $3 d \mathcal{N}=2$ theory of one chiral field $X$. Again, such dualities were skipped in [37] because of their abundance, where for brevity only the first steps of the reduction procedure from $4 d$ SCIs to $3 d$ partition functions were considered explicitly. The identities presented in this section lie further in the reduction hierarchy of EHIs to the hyperbolic level.

The equality of partition functions considered in [61] (later also discussed in [30, 121]) is obtained as a reduction of the $V$-function identities as well [15]. The equality of statistical sums of the initial theory and the mirror dual is taken from [19], where it was proven using the Fourier transformation formula [41]. The partition function of the $3 d$ mass-deformed $T[S U(2)]$ SYM theory coincides with the integral $I I_{1,(2,2)}^{1}\left(\mu_{1}, \mu_{2}, \nu_{1}, \nu_{2} ; \lambda\right)$ from [15] (again we take $\omega_{1}=$ $\left.b, \omega_{2}=1 / b\right)$ :

$$
K\left(\mu_{1}, \mu_{2}, \nu_{1}, \nu_{2}, \lambda\right)=\int_{-\mathrm{i} \infty}^{\mathrm{i} \infty} \prod_{i=1}^{2} \gamma^{(2)}\left(\mu_{i}-z, \nu_{i}+z ; b, b^{-1}\right) e^{\pi \mathrm{i} \lambda z} d z
$$


where one should restrict the parameters to obtain the expression from [61] as follows

$$
\mu_{1}=\nu_{1}=\frac{b+1 / b}{4}-\frac{m}{2}+\mu, \quad \mu_{2}=\nu_{2}=\frac{b+1 / b}{4}-\frac{m}{2}-\mu, \quad \lambda=-4 \xi .
$$

Integral (7.25) has the transformation formula described in Theorem 5.6.17 of [15]:

$$
\begin{aligned}
& K\left(\mu_{1}, \mu_{2}, \nu_{1}, \nu_{2}, \lambda\right)=\widetilde{K}\left(\sigma_{1}, \ldots, \sigma_{4}\right) e^{\pi \mathrm{i}\left(4 \widetilde{\sigma}^{2}-2 \mu_{1} \mu_{2}-2 \nu_{1} \nu_{2}\right) / 2} \\
& \quad \times \gamma^{(2)}\left(\left( \pm \lambda-\mu_{1}-\mu_{2}-\nu_{1}-\nu_{2}\right) / 2+b+1 / b ; b, b^{-1}\right),
\end{aligned}
$$

where

$$
\begin{aligned}
& \widetilde{K}\left(\sigma_{1}, \ldots, \sigma_{4}\right)=\frac{1}{2} \int_{-\mathrm{i} \infty}^{\mathrm{i} \infty} \frac{\prod_{i=1}^{4} \gamma^{(2)}\left(\sigma_{i} \pm y ; b, b^{-1}\right)}{\gamma^{(2)}\left( \pm 2 y ; b, b^{-1}\right)} e^{-2 \pi \mathrm{i} y^{2}} d y, \\
& \sigma_{1,2}=\mu_{1,2}+\widetilde{\sigma}, \quad \sigma_{3,4}=\nu_{1,2}-\widetilde{\sigma}, \quad 4 \widetilde{\sigma}=\nu_{1}+\nu_{2}-\mu_{1}-\mu_{2}-\lambda .
\end{aligned}
$$

There is a transformation formula for the integral $\widetilde{K}$ described in Theorem 5.6.14 (for $n=1$ ) in 15$]$ :

$$
\widetilde{K}\left(\sigma_{1}, \ldots, \sigma_{4}\right)=\widetilde{K}\left(\rho_{1}, \ldots, \rho_{4}\right) \prod_{1 \leq i<j \leq 4} \gamma^{(2)}\left(\sigma_{i}+\sigma_{j} ; b, b^{-1}\right) e^{-\pi \mathrm{i}(b+1 / b) \xi}
$$

where $2 \xi=b+1 / b-\sum_{i=1}^{4} \sigma_{i}, \rho_{i}=\sigma_{i}+\xi, i=1,2,3,4$.

Combining together formula (7.26), symmetry transformation (7.28) and, finally, again (7.26) (taking into account that (17.27) is symmetric in all the parameters $\sigma_{i}$ ), one gets the symmetry transformation

$$
\begin{gathered}
K((b+1 / b) / 4-m / 2 \pm \mu,(b+1 / b) / 4-m / 2 \pm \mu,-4 \xi) \gamma^{(2)}\left(-m ; b, b^{-1}\right) \\
=K(m / 2 \pm \xi, m / 2 \pm \xi,-4 \mu) \gamma^{(2)}\left(m ; b, b^{-1}\right) .
\end{gathered}
$$

Generalizing to arbitrary parameters $\mu_{1}, \mu_{2}, \nu_{1}, \nu_{2}$ one obtains formula (A.31) from [19]. Described symmetry transformation formulas allow one to derive more identities apart from (7.29), which should be explored separately. Here our aim was to show that all known examples of the equalities of partition functions from the literature are obtained as reductions of the identities for EHIs (actually, here we have discussed only the reduction of the elliptic beta integral and the $V$-function). There is also an interesting connection of the partition function of mass-deformed $T[S U(2)]$ theory with the Liouville theory [61], where it coincides with the $S$-duality kernel connecting conformal blocks [122]. Note also that it can be derived from SCI of $4 d \mathcal{N}=2 \mathrm{SYM}$ theory with $S U(2)$ gauge group and 4 hypermultiplets [49].

We conclude this section by stating that the arguments given above are quite general and applied to any state integral model. Other examples for different knots presented in [59] are obtained from the reduction of SCIs of $4 d \mathcal{N}=1$ quiver supersymmetric field theories and coincide with the partition functions of $3 d \mathcal{N}=2$ theories in which one restricts fugacities associated with the matter content of the theory. The results of this section may be useful for a better understanding of a generalization of the AGT duality [2], connecting $4 d$ and $2 d$ theories, to the duality connecting $3 d \mathrm{CS}$ and $3 d \mathcal{N}=2$ supersymmetric field theories [29, 30, 38, 61].

\section{REDUCTION TO THE $2 d$ VORTEX PARTition FUnCtion}

Dimensional reductions of field theories are usually considered directly at the level of physical degrees of freedom. As discussed in the previous section, often it is easier to make such reductions at the level of collective objects such as partition or correlation functions and topological indices. In particular, partition functions of the field theories on the squashed threesphere $S_{b}^{3}$ can be derived from $4 d$ SCIs [37] (the case of ordinary $S^{3}$ corresponds to the limit 
$\left.\omega_{1}=\omega_{2}^{-1} \rightarrow 1\right)$. An obvious question is whether one can proceed further and reduce $3 d$ partition functions to $2 d$ statistical sums? The squashed three-sphere is isomorphic to $S^{2} \times S^{1}$ and by shrinking the radius of $S^{1}$ to zero one reduces this manifold to $S^{2}$, which is a two-dimensional space-time. One obtains in this way the vortex partition function for a $2 d$ supersymmetric sigma-model. This partition function is the object of recent active studies [13, 31, 51, 107, 126]. Its relation to the $3 d$ Omega background is discussed in [30]. From the mathematical point of view the $4 d / 3 d$ correspondence of [37] is described by the reduction of EHIs to the hyperbolic $q$-hypergeometric integrals (see, e.g., [27, 98]). Here we proceed with further reduction to the rational level [98] described by the integrals employing elementary functions and the standard gamma function. In [86, it was found that introducing into $4 d$ SCI of the surface operators leads to the $2 d(4,4)$ SCFT coupled to the $4 d$ theory; here we obtain a more complete $2 d$ picture. A different type of $2 d$ partition function associated with SCIs of $\mathcal{N}=2$ theories was considered recently in [48]. A new $2 d / 3 d / 4 d$ correspondence has been discovered in [115], where it was shown that both $4 d$ SCIs and $3 d$ partition functions of supersymmetric quiver theories describe statistical sums of certain integrable models of $2 d$ Ising-like spin systems with continuous values of spins.

Let us discuss first the reduction of $4 d$ SCIs to $3 d$ partition functions on the example of Intriligator-Pouliot duality [65]. As shown in Sect. 2 above and in [36], one can derive SCIs of $4 d \mathcal{N}=1 \mathrm{SYM}$ theories with the orthogonal gauge groups from the corresponding $S P(2 N)$ SCIs. But we can reduce the latter $4 d$ SCIs to $3 d$ partition functions along the lines of [37]. This results in $3 d$ dualities for both SYM [1] and CS [52] theories and both $S P(2 N)$ and $U(N)$ gauge groups. We stress that $4 d$ SCIs and dualities are defined as a rule by unique relations for EHIs, and at the $3 d$-level one obtains the whole web of dualities/SCIs both for SYM and CS theories based on different gauge groups.

More technically, we start from integral (2.1) describing SCI of the electric theory of [65] [36, 117]. Reducing it to the hyperbolic level [27, 98, one finds the following integral (2.1) [15]:

$$
\begin{aligned}
Z= & \frac{1}{N !} \int_{\mathcal{C}^{N}} \prod_{1 \leq i<j \leq N} \frac{1}{\gamma^{(2)}\left( \pm\left(z_{i}-z_{j}\right) ; \omega_{1}, \omega_{2}\right)} \\
& \times e^{2 \pi \mathrm{i}(\lambda+1 / 2)\left(\omega_{1}+\omega_{2}\right) \sum_{j=1}^{N} z_{j} / \omega_{1} \omega_{2}} \prod_{i, j=1}^{N} \gamma^{(2)}\left(\mu_{i}-z_{j}, \nu_{i}+z_{j} ; \omega_{1}, \omega_{2}\right) \prod_{j=1}^{N} \frac{d z_{j}}{\mathrm{i} \sqrt{\omega_{1} \omega_{2}}},
\end{aligned}
$$

where $\mathcal{C}$ is the Mellin-Barnes type integration contour.

In [124, Willett and Yakov showed that this integral describes the partition function [56, 68] of the electric theory for Aharony duality [1], which is a $3 d \mathcal{N}=2 \mathrm{SYM}$ theory living on the squashed three-sphere with $U(N)$ gauge group, $N_{f}=N$ left quarks forming the fundamental representation of $U(N), N_{f}=N$ right quarks forming the antifundamental representation of $U(N)$, and additional singlets $V_{ \pm}$. In (8.1), parameters $z_{j}, j=1, \ldots, N$, are the fugacities associated with the gauge group $U(N), \lambda$ is associated with the Fayet-Illiopoulos term (the coefficient $4(\lambda+1 / 2)\left(\omega_{1}+\omega_{2}\right)$ is introduced for convenience). Parameters $\mu_{i}, \nu_{i}, i=1, \ldots, N$, are the fugacities of $S U(N) \times S U(N)$ non-abelian global symmetry group, which are normalized by taking into account the abelian part of the global symmetry $U(1)_{A} \times U(1)_{J} \times U(1)_{R}$.

Consider the limit $\omega_{2} \rightarrow \infty$ using the hyperbolic gamma function asymptotics

$$
\gamma^{(2)}\left(z ; \omega_{1}, \omega_{2}\right) \underset{\omega_{2} \rightarrow \infty}{=}\left(\frac{\omega_{2}}{2 \pi \omega_{1}}\right)^{\frac{1}{2}-z} \frac{\Gamma_{r a t}\left(z / \omega_{1}\right)}{\sqrt{2 \pi}} .
$$


The $3 d$ partition function (8.1) then reduces to

$$
Z^{l i m}=\frac{\omega_{2}^{N / 2}}{N ! \omega_{1}^{3 N / 2}}\left(\frac{\omega_{2}}{\omega_{1}}\right)^{-\sum_{i=1}^{N}\left(\mu_{i}+\nu_{i}\right)} Z^{\text {vortex }}
$$

where $Z^{\text {vortex }}$ is the function appearing after formula (2.6) in [51] for $N_{f}=N$ :

$$
Z^{\text {vortex }}=\int_{\mathcal{C}^{N}} \frac{e^{2 \pi \mathrm{i}(\lambda+1 / 2) \sum_{j=1}^{N} \frac{z_{j}}{\omega_{1}}}}{\prod_{1 \leq i<j \leq N} \Gamma_{\text {rat }}\left(\frac{z_{i}-z_{j}}{\omega_{1}}, \frac{z_{j}-z_{i}}{\omega_{1}}\right)} \prod_{i, j=1}^{N} \Gamma_{\text {rat }}\left(\frac{\mu_{i}-z_{j}}{\omega_{1}}, \frac{\nu_{i}+z_{j}}{\omega_{1}}\right) \prod_{j=1}^{N} \frac{d z_{j}}{2 \pi \mathrm{i}} .
$$

The multiplier $\prod_{i \neq j} \Gamma_{r a t}\left(\left(a_{i}-a_{j}\right) / \omega_{1}\right)$ standing in front of the integral in [51] is not relevant for our discussion and is omitted.

Expression (8.3) defines the vortex partition function for $2 d(2,2)$ supersymmetric field theory with $U(N)$ gauge group and $N_{f}=N$ flavors. Its representation as a sum over Young diagrams can be obtained from the partition function of $4 d \mathcal{N}=2$ SYM theory [88, 89] in the limit $\omega_{2} \rightarrow \infty$ [107]. More precisely, in this limit one should also normalize the variable associated with the instanton parameter to compensate additional divergences emerging for $\omega_{2} \rightarrow \infty$. In [51, it was realized that the latter sum over Young diagrams (instantons) can be rewritten as a single contour integral (8.3), which leads to a better understanding of this function from the mathematical point of view.

This observation can be generalized to any number of flavors $N_{f}$ appearing in [51] by starting from the partition function for $3 d \mathcal{N}=2 \mathrm{SYM}$ theory with $U(N)$ gauge group, $N_{f} \neq N$ flavors, and looking at the same limit $\omega_{2} \rightarrow \infty$ accompanied by pulling some of the parameters to infinity (i.e., by integrating out some of the quarks). Technically, one should use the asymptotic expansion of the gamma function $\Gamma_{\text {rat }}(x) \rightarrow \sqrt{2 \pi} e^{-x} x^{x-1 / 2}$ for $x \rightarrow \infty$. In principle one can get in the same manner the vortex partition functions for $2 d$ supersymmetric field theories with symplectic and orthogonal gauge groups and different matter fields (the contribution of adjoint matter field was considered in [13]).

We conclude by several remarks on the importance of the observation made in this section. First, it may be very useful for checking a $2 d$ analog of Seiberg's duality which was recently proposed and studied in [60, 109]. Second, this reduction is close to the one studied in the literature on connections of $3 d$ Chern-Simons theories with $2 d$ supersymmetric field theories [31] linking vortex partition function to the BPS invariants of dual geometries. Finally, perhaps the most important, $4 d$ SCIs for $\mathcal{N}=1$ SYM theories are connected to $4 d$ partition functions for $\mathcal{N}=2 \mathrm{SYM}$ theories in the discussed above limit.

\section{Conclusion}

In [116, 117], we initiated the classification of EHIs on different root systems and described all known examples of such integrals for $A_{N}, B C_{N}$, and $G_{2}$ root systems in association with $\mathcal{N}=1$ supersymmetric dualities. In [119], for all irreducible root systems we described such integrals associated with $\mathcal{N}=4 \mathrm{SYM}$ theories; there are also two more particular examples associated with $\mathcal{N}=1 \mathrm{SYM} E_{6}$ and $F_{4}$ gauge group theories.

In the present paper we have described all known cases when $B C_{n}$-EHIs and corresponding physical dualities with the symplectic gauge groups are reduced to SCIs/dualities for orthogonal groups by a restriction of parameters entering the integrals. Remarkably, there are EHIs for the $B_{N}$ and $D_{N}$ root systems which (currently) cannot be obtained from integrals on the $B C_{N}$ root system - they come from SCIs for $\mathcal{N}=1 \mathrm{SYM} S O(2 N+1)$ or $S O(2 N)$ gauge group theories with the matter fields in spinor representation. Description of this type of integrals is one of the 
main results of the present paper. Physical dualities of the corresponding gauge theories lead to the conjectures on the equality of respective SCIs. The latter conjectural identities for EHIs use characters of the spinor representations, and they were not predicted by the mathematical developments prior to the supersymmetric duality ideas intervention. All of them require now rigorous mathematical proofs.

In addition to SCIs for $\mathcal{N}=1$ dualities considered in this paper, one can investigate SCIs for electric-magnetic dualities for extended supersymmetric field theories: the quiver $\mathcal{N}=2$ SYM theories with $S O / S P$ gauge groups [119] or the $S P / S O$-groups duality [54] in $\mathcal{N}=4$ SYM theory [46, 119]. Note that SCIs for extended supersymmetric theories can be obtained from SCIs of $\mathcal{N}=1$ theories by adjusting the matter content appropriately together with the hypercharges, as described in [117, 119]. In the field theory lagrangians one should fix also appropriately the superpotentials.

As described in [117], one of the physical applications of the EHI identities uses the reduction $p=q=0$, which yields the Hilbert series counting gauge invariant operators [57, 96]. Another interesting application of our identities is connected with the Seiberg type dualities for $3 d$ super-Yang-Mills and Chern-Simons theories with orthogonal gauge groups. Derivation of $3 d$ partition functions out of $4 d$ SCIs of [37] yields the most efficient way of obtaining $3 d$-dualities. Technically, the reduction to $3 d$ theories is obtained after the parametrization in $4 d$ SCIs of the integration variables, global symmetry fugacities, and bases $p$ and $q$ similar to (7.13), with the subsequent limit $r \rightarrow 0$. As a result, $4 d$ SCIs defined on $S^{3} \times S^{1}$ reduce to partition functions on the squashed three-sphere $S_{b}^{3}$ [56, 68]. In this limit the elliptic gamma function is reduced to the hyperbolic gamma function. It is thus natural to expect that all the dualities considered in [69] can be recovered by a reduction from the $4 d$ SCIs considered in the present paper. A more detailed description of the resulting hyperbolic integrals was given in Sect. 7. The reduction procedure for $3 d$ theories from $S P(2 N)$ to $S O(n)$ gauge group is similar to the one in $4 d$ theories without spinor matter. For that one needs the duplication formula for the hyperbolic gamma function

$$
\gamma^{(2)}\left(2 z ; \omega_{1}, \omega_{2}\right)=\gamma^{(2)}\left(z, z+\omega_{1} / 2, z+\omega_{2} / 2, z+\left(\omega_{1}+\omega_{2}\right) / 2 ; \omega_{1}, \omega_{2}\right) .
$$

To get $S O(2 N+1)$ partition functions it is necessary to restrict three chemical potentials to $\omega_{1} / 2, \omega_{2} / 2,\left(\omega_{1}+\omega_{2}\right) / 2$ (or two chemical potentials to $\left.\omega_{1} / 2, \omega_{2} / 2\right)$ and for $S O(2 N)$ case one should fix four chemical potentials equal to $0, \omega_{1} / 2, \omega_{2} / 2,\left(\omega_{1}+\omega_{2}\right) / 2$ (or three chemical potentials equal to $\left.0, \omega_{1} / 2, \omega_{2} / 2\right)$. This leads to a variety of $3 d \mathcal{N}=2$ supersymmetric dual theories (both SYM and CS theories) without spinor matter. To construct $3 d$ dualities for theories with the spinor matter one should follow the algorithm suggested in [37]. As a final mathematical remark, we stress that all our computations are performed analytically, i.e. we described exact (conjectural or proven) equalities of the compared functions in all admissible domains of values of the parameters.

Acknowledgments. We dedicate this work to D. I. Kazakov on the occasion of his 60th birthday with the wishes of further scientific successes. We are indebted to G. E. Arutyunov, A. A. Belavin, T. Dimofte, F. A. H. Dolan, S. A. Frolov, S. Gukov, A. V. Litvinov, I. V. Melnikov, and A. F. Oskin for valuable discussions. We thank the referee for drawing our attention to papers [82, 90]. G. V. would like to thank H. Nicolai for general support and the Universities of Minnesota, Chicago, and Utrecht, CERN, DESY, Nordita, and the Niels Bohr Institute in Copenhagen for invitations and warm hospitality during visits to these Institutes. V. S. was partially supported by the RFBR grants 09-01-93107-NCNIL-a and 11-01-00980, 
NRU HSE Academic Fund Program (grant no. 12-09-0064) and by the Max Planck Institute for mathematics. This work is supported also by the Heisenberg-Landau program.

\section{Appendix A. Characters of Representations of orthogonal groups}

In this Appendix we describe characters of representations of orthogonal groups used in the paper. For needed $S U(N)$ and $S P(2 N)$ group characters, see Appendix A of [117], and invariant measures for all classical groups are listed in Appendix B of that paper.

$S O(N)$-Groups with even and odd $N$ have substantially different properties and should be considered separately. The characters for their spinor representations are described most conveniently by the expressions involving square roots of $z_{j}$-variables which are not analytical. To overcome this obstacle we just double the root lengths which results in the replacement in characters variables $z_{j}$ by $z_{j}^{2}$ and assume in the integrals that $z_{j}$ lie on the unit circle with positive orientation. We remark that the adjoint representation for orthogonal groups coincides with the $T_{A}$-representation.

$S O(2 N)$ group. The characters are expressed in terms of $N$ independent variables $z_{i}, i=$ $1, \ldots, N$. For the fundamental representation one has

$$
\chi_{f, S O(2 N)}=\sum_{i=1}^{N} z_{i}^{ \pm 1} \equiv \sum_{i=1}^{N}\left(z_{i}+z_{i}^{-1}\right) .
$$

The $T_{S}$-representation character is

$$
\chi_{T_{S}, S O(2 N)}=\sum_{1 \leq i<j \leq N} z_{i}^{ \pm 1} z_{j}^{ \pm 1}+\sum_{i=1}^{N} z_{i}^{ \pm 2}+N-1,
$$

the $T_{A}$-representation character is

$$
\chi_{T_{A}, S O(2 N)}=\sum_{1 \leq i<j \leq N} z_{i}^{ \pm 1} z_{j}^{ \pm 1}+N .
$$

The needed spinor representation characters are listed case by case. For $S O(2 N)$ groups there are two types of inequivalent spinors, denoted as $s$ and $c$. For $S O(8)$, the spinor representation $s$ and $c$ are 8-dimensional, self-conjugate, and their characters have the form

$$
\chi_{s, S O(8)}=z^{ \pm 1}+z^{-1} \sum_{1 \leq i<j \leq 4} z_{i} z_{j}
$$

where $z=\sqrt{z_{1} z_{2} z_{3} z_{4}}$. For $S O(10)$, the $s$-representation is 16-dimensional, it is complex conjugate to $c$ (so that the character for $c$ can be obtained from the $s$-character by the substitution $z \rightarrow 1 / z)$. Its character is

$$
\chi_{s, S O(10)}=z+z^{-1} \sum_{j=1}^{5} z_{j}+z \sum_{1 \leq i<j \leq 5} z_{i}^{-1} z_{j}^{-1},
$$

where $z=\sqrt{z_{1} z_{2} z_{3} z_{4} z_{5}}$. For $S O(12)$, the $s$ - and $c$-representations are 32-dimensional, selfconjugate, and have the character

$$
\chi_{s, S O(12)}=z^{ \pm 1}+z^{-1} \sum_{j=1}^{6} z_{j}+z \sum_{j=1}^{6} z_{j}^{-1},
$$


where $z=\sqrt{z_{1} z_{2} z_{3} z_{4} z_{5} z_{6}}$. For $S O(14)$, the $s$-representation is 64-dimensional, it is complexconjugate to $c$, and its character is (with $z=\sqrt{z_{1} z_{2} z_{3} z_{4} z_{5} z_{6} z_{7}}$ )

$$
\chi_{s, S O(14)}=z+z^{-1} \sum_{j=1}^{7} z_{j}+z \sum_{1 \leq i<j \leq 7} z_{i}^{-1} z_{j}^{-1}+z^{-1} \sum_{1 \leq i<j<k \leq 7} z_{i} z_{j} z_{k} .
$$

$S O(2 N+1)$ group. All the characters are expressed in terms of $N$ independent variables $z_{i}, i=1, \ldots, N$. The fundamental representation character is

$$
\chi_{f, S O(2 N+1)}=\sum_{i=1}^{N} z_{i}^{ \pm 1}+1 .
$$

The character for $T_{S}$-representation is

$$
\chi_{T_{S}, S O(2 N+1)}=\sum_{1 \leq i<j \leq N} z_{i}^{ \pm 1} z_{j}^{ \pm 1}+\sum_{i=1}^{N} z_{i}^{ \pm 2}+\sum_{i=1}^{N} z_{i}^{ \pm 1}+N,
$$

the character for the $T_{A}$-representation is

$$
\chi_{T_{A}, S O(2 N+1)}=\sum_{1 \leq i<j \leq N} z_{i}^{ \pm 1} z_{j}^{ \pm 1}+\sum_{i=1}^{N} z_{i}^{ \pm 1}+N .
$$

The spinor representation characters are given for the lowest rank groups only. For $S O(7)$, the spinor representation is 8-dimensional and its character is

$$
\chi_{s, S O(7)}=z^{ \pm 1}+z^{-1} \sum_{j=1}^{3} z_{j}+z \sum_{j=1}^{3} z_{j}^{-1},
$$

where $z=\sqrt{z_{1} z_{2} z_{3}}$. For $S O(9)$, the spinor representation is 16-dimensional and its character is

$$
\chi_{s, S O(9)}=z^{ \pm 1}+z^{-1} \sum_{j=1}^{4} z_{j}+z \sum_{j=1}^{4} z_{j}^{-1}+z^{-1} \sum_{1 \leq i<j \leq 4} z_{i} z_{j}
$$

where $z=\sqrt{z_{1} z_{2} z_{3} z_{4}}$. For $S O(11)$, the spinor representation is 32-dimensional and its character is

$$
\chi_{s, S O(11)}=z^{ \pm 1}+z^{-1} \sum_{j=1}^{5} z_{j}+z \sum_{j=1}^{5} z_{j}^{-1}+z^{-1} \sum_{1 \leq i<j \leq 5} z_{i} z_{j}+z \sum_{1 \leq i<j \leq 5}\left(z_{i} z_{j}\right)^{-1}
$$

where $z=\sqrt{z_{1} z_{2} z_{3} z_{4} z_{5}}$. For $S O(13)$, the spinor representation is 64-dimensional and its character is (with $z=\sqrt{z_{1} z_{2} z_{3} z_{4} z_{5} z_{6}}$ )

$\chi_{s, S O(13)}=z^{ \pm 1}+z^{-1} \sum_{j=1}^{6} z_{j}+z \sum_{j=1}^{6} z_{j}^{-1}+z^{-1} \sum_{1 \leq i<j \leq 6} z_{i} z_{j}+z \sum_{1 \leq i<j \leq 6}\left(z_{i} z_{j}\right)^{-1}+z^{-1} \sum_{1 \leq i<j<k \leq 6} z_{i} z_{j} z_{k}$. 


\section{REFERENCES}

[1] O. Aharony, IR duality in $d=3 \mathcal{N}=2$ supersymmetric $U S p\left(2 N_{c}\right)$ and $U\left(N_{c}\right)$ gauge theories, Phys. Lett. B404 (1997), 71-76.

[2] L. F. Alday, D. Gaiotto, and Y. Tachikawa, Liouville Correlation Functions from Four-dimensional Gauge Theories, Lett. Math. Phys. 91 (2010), 167-197.

[3] G. E. Andrews, R. Askey, and R. Roy, Special Functions, Encyclopedia of Math. Appl. 71, Cambridge Univ. Press, Cambridge, 1999.

[4] H. Awata, B. Feigin, A. Hoshino, M. Kanai, J. Shiraishi, and S. Yanagida, Notes on Ding-Iohara algebra and AGT conjecture, RIMS Kokyuroku 1765 (2011), 12-32.

[5] H. Awata, H. Kubo, S. Odake, and J. Shiraishi, A quantum deformation of the Virasoro algebra and the Macdonald symmetric functions, Lett. Math. Phys. 38 (1996), 33-51.

[6] H. Awata and Y. Yamada, Five-dimensional AGT Conjecture and the Deformed Virasoro Algebra, JHEP 1001 (2010) 125.

[7] D. Bashkirov and A. Kapustin, Dualities between $\mathcal{N}=8$ superconformal field theories in three dimensions, JHEP 1105 (2011) 074.

[8] V. V. Bazhanov and S. M. Sergeev, A master solution of the quantum Yang-Baxter equation and classical discrete integrable equations, ATMP 16 (2012), 65-95.

[9] S. Benvenuti, B. Feng, A. Hanany, and Y. H. He, Counting BPS Operators in Gauge Theories: Quivers, Syzygies and Plethystics, JHEP 0711 (2007) 050.

[10] S. Benvenuti and S. Pasquetti, 3D-partition functions on the sphere: exact evaluation and mirror symmetry, JHEP 1205 (2012) 099.

[11] M. Berkooz, P. L. Cho, P. Kraus, and M. J. Strassler, Dual descriptions of SO(10) SUSY gauge theories with arbitrary numbers of spinors and vectors, Phys. Rev. D56 (1997), 7166-7182.

[12] M. Bianchi, F. A. Dolan, P. J. Heslop, and H. Osborn, $\mathcal{N}=4$ superconformal characters and partition functions, Nucl. Phys. B767 (2007), 163-226.

[13] G. Bonelli, A. Tanzini, and J. Zhao, Vertices, Vortices and Interacting Surface Operators, JHEP 1206 (2012) 178; The Liouville side of the Vortex, JHEP 1109 (2011) 096.

[14] J. H. Brodie and M. J. Strassler, Patterns of duality in $\mathcal{N}=1$ SUSY gauge theories, or: Seating preferences of theater going nonAbelian dualities, Nucl. Phys. B524 (1998), 224-250.

[15] F. J. van de Bult, Hyperbolic hypergeometric functions, Ph. D. thesis, University of Amsterdam, 2007.

[16] F. J. van de Bult, An elliptic hypergeometric integral with $W\left(F_{4}\right)$ symmetry, Ramanujan J. 25 (1) (2011), $1-20$.

[17] F. J. van de Bult, E. M. Rains, and J. V. Stokman, Properties of generalized univariate hypergeometric functions, Commun. Math. Phys. 275 (2007), 37-95.

[18] A. Bytsko and J. Teschner, R-operator, co-product and Haar-measure for the modular double of $U_{q}($ sl $(2, R))$, Commun. Math. Phys. 240 (2003), 171-196.

[19] A. G. Bytsko and J. Teschner, Quantization of models with non-compact quantum group symmetry: Modular XXZ magnet and lattice sinh-Gordon model, J. Phys. A39 (2006), 12927-12981.

[20] P. L. Cho, More on chiral-nonchiral dual pairs, Phys. Rev. D56 (1997), 5260-5271.

[21] N. Craig, R. Essig, A. Hook, and G. Torroba, New dynamics and dualities in supersymmetric chiral gauge theories, JHEP 09 (2011) 046.

[22] C. Csáki and H. Murayama, New confining $\mathcal{N}=1$ supersymmetric gauge theories, Phys. Rev. D59 (1999) 065001.

[23] C. Csaki, M. Schmaltz, and W. Skiba, Confinement in $\mathcal{N}=1$ SUSY gauge theories and model building tools, Phys. Rev. D55 (1997), 7840-7858.

[24] C. Csáki, M. Schmaltz, W. Skiba, and J. Terning, Selfdual $\mathcal{N}=1$ SUSY gauge theories, Phys. Rev. D56 (1997), 1228-1238.

[25] J. F. van Diejen and V. P. Spiridonov, An elliptic Macdonald-Morris conjecture and multiple modular hypergeometric sums, Math. Res. Lett 7 (2000), 729-746.

[26] J. F. van Diejen and V. P. Spiridonov, Elliptic Selberg integrals, Internat. Math. Res. Notices, no. 20 (2001), $1083-1110$.

[27] J. F. van Diejen and V. P. Spiridonov, Unit circle elliptic beta integrals, Ramanujan J. 10 (2005), $187-204$.

[28] T. Dimofte, Quantum Riemann Surfaces in Chern-Simons Theory, arXiv:1102.4847 [hep-th]. 
[29] T. Dimofte, D. Gaiotto, and S. Gukov, Gauge Theories Labelled by Three-Manifolds, arXiv:1108.4389 [hep-th].

[30] T. Dimofte and S. Gukov, Chern-Simons Theory and S-duality, arXiv:1106.4550 [hep-th].

[31] T. Dimofte, S. Gukov, and L. Hollands, Vortex Counting and Lagrangian 3-manifolds, Lett. Math. Phys. 98 (2011) 225-287.

[32] T. Dimofte, S. Gukov, J. Lenells, and D. Zagier, Exact Results for Perturbative Chern-Simons Theory with Complex Gauge Group, Commun. Num. Theor. Phys. 3 (2009), 363-443.

[33] J. Distler and A. Karch, $\mathcal{N}=1$ dualities for exceptional gauge groups and quantum global symmetries, Fortsch. Phys. 45 (1997), 517-533.

[34] V. K. Dobrev and V. B. Petkova, On The Group Theoretical Approach To Extended Conformal Supersymmetry: Classification Of Multiplets, Lett. Math. Phys. 9 (1985), 287-298.

[35] F. A. Dolan, Counting BPS operators in $\mathcal{N}=4 S Y M$, Nucl. Phys. B790 (2008), 432-464.

[36] F. A. Dolan and H. Osborn, Applications of the Superconformal Index for Protected Operators and qHypergeometric Identities to $\mathcal{N}=1$ Dual Theories, Nucl. Phys. B818 (2009), 137-178.

[37] F. A. H. Dolan, V. P. Spiridonov, and G. S. Vartanov, From 4d superconformal indices to $3 d$ partition functions, Phys. Lett. B704 (2011), 234-241.

[38] N. Drukker, D. Gaiotto, and J. Gomis, The Virtue of Defects in $4 D$ Gauge Theories and 2D CFTs, JHEP 1106 (2011), 025.

[39] N. Drukker, M. Marino, and P. Putrov, From weak to strong coupling in ABJM theory, Commun. Math. Phys. 306 (2011), 511-563.

[40] L. D. Faddeev, Discrete Heisenberg-Weyl group and modular group, Lett. Math. Phys. 34 (1995), 249-254.

[41] L. D. Faddeev, R. M. Kashaev, and A. Y. Volkov, Strongly coupled quantum discrete Liouville theory. 1. Algebraic approach and duality, Commun. Math. Phys. 219 (2001), 199-219.

[42] L. Faddeev and A. Yu. Volkov, Abelian current algebra and the Virasoro algebra on the lattice, Phys. Lett. B315 (1993), 311-318.

[43] B. Feigin, K. Hashizume, A. Hoshino, J. Shiraishi, and S. Yanagida, A commutative algebra on degenerate $C P^{1}$ and Macdonald polynomials, J. Math. Phys. 50 (2009) 095215.

[44] B. Feng, A. Hanany, and Y. H. He, Counting gauge invariants: the plethystic program, JHEP 0703 (2007) 090.

[45] G. Festuccia and N. Seiberg, Rigid Supersymmetric Theories in Curved Superspace, JHEP 1106 (2011) 114.

[46] A. Gadde, E. Pomoni, L. Rastelli, and S. S. Razamat, S-duality and 2d Topological QFT, JHEP 03 (2010) 032.

[47] A. Gadde, L. Rastelli, S. S. Razamat, and W. Yan, The Superconformal Index of the $E_{6} S C F T$, JHEP 08 (2010) 107.

[48] A. Gadde, L. Rastelli, S. S. Razamat and W. Yan, The 4d Superconformal Index from q-deformed $2 d$ Yang-Mills, Phys. Rev. Lett. 106 (2011) 241602.

[49] A. Gadde and W. Yan, Reducing the 4d Index to the $S^{3}$ Partition Function, JHEP 2012 (2012) 003.

[50] D. Gaiotto and E. Witten, Knot Invariants from Four-Dimensional Gauge Theory, arXiv:1106.4789 [hep-th].

[51] A. A. Gerasimov and D. R. Lebedev, On topological field theory representation of higher analogs of classical special functions, JHEP 1109 (2011) 076.

[52] A. Giveon and D. Kutasov, Seiberg Duality in Chern-Simons Theory, Nucl. Phys. B812 (2009), 1-11.

[53] D. Green, Z. Komargodski, N. Seiberg, Y. Tachikawa, and B. Wecht, Exactly Marginal Deformations and Global Symmetries, JHEP 1006 (2010) 106.

[54] P. Goddard, J. Nuyts, and D. I. Olive, Gauge Theories And Magnetic Charge, Nucl. Phys. B125 (1977), $1-28$.

[55] R. A. Gustafson, Some q-beta integrals on $S U(n)$ and $S p(n)$ that generalize the Askey-Wilson and Nassrallah-Rahman integrals, SIAM J. Math. Anal. 25 (1994), 441-449.

[56] N. Hama, K. Hosomichi, and S. Lee, Notes on SUSY gauge theories on three-sphere, JHEP 1103 (2011) 127; SUSY gauge theories on squashed three-spheres, JHEP 1105 (2011) 014.

[57] A. Hanany and N. Mekareeya, Counting gauge invariant operators in SQCD with classical gauge groups, JHEP 0810 (2008) 012.

[58] K. Hikami, Hyperbolic Structure Arising from a Knot Invariant, Int. J. Mod. Phys. A16 (2001), 3309-3333. 
[59] K. Hikami, Generalized Volume Conjecture and the A-Polynomials - the Neumann-Zagier Potential Function as a Classical Limit of Quantum Invariant, J. Geom. Phys. 57 (2007), 1895-1940.

[60] K. Hori, Duality In Two-Dimensional (2,2) Supersymmetric Non-Abelian Gauge Theories, arXiv:1104.2853 [hep-th].

[61] K. Hosomichi, S. Lee, and J. Park, AGT on the S-duality Wall, JHEP 1012 (2010) 079.

[62] Y. Imamura, Relation between the $4 d$ superconformal index and the $S^{3}$ partition function, JHEP 1109 (2011) 133.

[63] Y. Imamura and S. Yokoyama, Index for three dimensional superconformal field theories with general $R$ charge assignments, JHEP 1104 (2011) 007.

[64] K. Intriligator, New RG fixed points and duality in supersymmetric $S P(N)$ and $S O(N)$ gauge theories, Nucl. Phys. B448 (1995), 187-198.

[65] K. A. Intriligator and P. Pouliot, Exact superpotentials, quantum vacua and duality in supersymmetric $S P(N)$ gauge theories, Phys. Lett. B353 (1995), 471-476.

[66] K. A. Intriligator and N. Seiberg, Duality, monopoles, dyons, confinement and oblique confinement in supersymmetric $S O(N)$ gauge theories, Nucl. Phys. B444 (1995), 125-160.

[67] K. A. Intriligator and N. Seiberg, Lectures on supersymmetric gauge theories and electric-magnetic duality, Nucl. Phys. Proc. Suppl. 45BC (1996), 1-28.

[68] D. L. Jafferis, The exact superconformal R-symmetry extremizes $Z$, JHEP 1205 (2012) 159.

[69] A. Kapustin, Seiberg-like duality in three dimensions for orthogonal gauge groups, arXiv:1104.0466 [hep-th].

[70] A. Kapustin and B. Willett, Generalized Superconformal Index for Three Dimensional Field Theories, arXiv:1106.2484 [hep-th].

[71] A. Kapustin, B. Willett, and I. Yaakov, Exact results for Wilson loops in superconformal Chern-Simons theories with matter, JHEP 1003 (2010) 089.

[72] A. Karch, More on $\mathcal{N}=1$ selfdualities and exceptional gauge groups, Phys. Lett. B405 (1997), 280-286.

[73] T. Kawano, Duality of $\mathcal{N}=1$ Supersymmetric $S O(10)$ Gauge Theory with Matter in the Spinorial Representation, Prog. Theor. Phys. 95 (1996), 963-968.

[74] A. Khmelnitsky, Interpreting multiple dualities conjectured from superconformal index identities, JHEP 1003 (2010) 065.

[75] S. Kim, The complete superconformal index for $\mathcal{N}=6$ Chern-Simons theory, Nucl. Phys. B821 (2009), $241-284$.

[76] J. Kinney, J. M. Maldacena, S. Minwalla, and S. Raju, An index for 4 dimensional super conformal theories, Commun. Math. Phys. 275 (2007), 209-254.

[77] M. Klein, More confining $\mathcal{N}=1$ SUSY gauge theories from nonAbelian duality, Nucl. Phys. B553 (1999), $155-204$.

[78] C. Krattenthaler, V. P. Spiridonov, and G. S. Vartanov, Superconformal indices of three-dimensional theories related by mirror symmetry, JHEP 06 (2011) 008.

[79] R. G. Leigh and M. J. Strassler, Duality of $S p\left(2 N_{c}\right)$ and $S O\left(N_{c}\right)$ supersymmetric gauge theories with adjoint matter, Phys. Lett. B356 (1995), 492-499.

[80] S. L. Lukyanov and Y. Pugai, Bosonization of ZF algebras: Direction toward deformed Virasoro algebra, J. Exp. Theor. Phys. 82 (1996), 1021-1045.

[81] N. Maru, Confining phase in SUSY SO(12) gauge theory with one spinor and several vectors, Mod. Phys. Lett. A13 (1998), 1361-1370.

[82] G. W. Moore, N. Nekrasov, and S. Shatashvili, D-particle bound states and generalized instantons, Commun. Math. Phys. 209 (2000), 77-95 .

[83] Y. Nakayama, Index for orbifold quiver gauge theories, Phys. Lett. B636 (2006), 132-136.

[84] Y. Nakayama, Index for supergravity on $A d S_{5} \times T^{1,1}$ and conifold gauge theory, Nucl. Phys. B755 (2006), 295-312.

[85] Y. Nakayama, Finite $N$ index and angular momentum bound from gravity, Gen. Rel. Grav. 39 (2007), $1625-1638$.

[86] Y. Nakayama, $4 D$ and $2 D$ superconformal index with surface operator, JHEP 1108 (2011) 084.

[87] S. Nawata, Localization of $\mathcal{N}=4$ Superconformal Field Theory on $S^{1} \times S^{3}$ and Index, JHEP 2011 (2011) 144.

[88] N. A. Nekrasov, Seiberg-Witten prepotential from instanton counting, Adv. Theor. Math. Phys. 7 (2003), $831-864$. 
[89] N. Nekrasov and A. Okounkov, Seiberg-Witten theory and random partitions, The Unity of Mathematics, Progr. Math. 244, Birkhauser, Boston, MA, 2006, pp. 525-596.

[90] N. Nekrasov and S. Shadchin, ABCD of instantons, Commun. Math. Phys. 252 (2004), 359-391.

[91] H. Osborn, Topological Charges For $\mathcal{N}=4$ Supersymmetric Gauge Theories And Monopoles Of Spin 1, Phys. Lett. B83 (1979), 321-326.

[92] V. Pestun, Localization of gauge theory on a four-sphere and supersymmetric Wilson loops, Commun. Math. Phys. 313 (2012), 71-129.

[93] P. Pouliot, Chiral duals of nonchiral SUSY gauge theories, Phys. Lett. B359 (1995), 108-113.

[94] P. Pouliot and M. J. Strassler, A Chiral SU(N) Gauge Theory and its Non-Chiral Spin(8) Dual, Phys. Lett. B370 (1996), 76-82.

[95] P. Pouliot and M. J. Strassler, Duality and Dynamical Supersymmetry Breaking in Spin(10) with a Spinor, Phys. Lett. B375 (1996), 175-180.

[96] P. Pouliot, Molien function for duality, JHEP 9901 (1999) 021.

[97] E. M. Rains, Transformations of elliptic hypergeometric integrals, Ann. Math. 171 (2010), 169-243.

[98] E. M. Rains, Limits of elliptic hypergeometric integrals, Ramanujan J. 18 (3) (2009), 257-306.

[99] C. Römelsberger, Counting chiral primaries in $\mathcal{N}=1, d=4$ superconformal field theories, Nucl. Phys. B747 (2006), 329-353.

[100] C. Römelsberger, Calculating the superconformal index and Seiberg duality, arXiv:0707.3702 [hep-th].

[101] S. N. M. Ruijsenaars, First order analytic difference equations and integrable quantum systems, J. Math. Phys. 38 (1997), 1069-1146.

[102] R. Schiappa and N. Wyllard, An A threesome: Matrix models, $2 d$ CFTs and $4 d \mathcal{N}=2$ gauge theories, J. Math. Phys. 51 (2010) 082304.

[103] N. Seiberg, Exact results on the space of vacua of four-dimensional SUSY gauge theories, Phys. Rev. D49 (1994), 6857-6863.

[104] N. Seiberg, Electric-magnetic duality in supersymmetric non-Abelian gauge theories, Nucl. Phys. B435 (1995), 129-146.

[105] N. Seiberg, Recent advances in supersymmetry, talk at the conference "Strings-2011" (Upsalla, June 2011), http://media.medfarm.uu.se/flvplayer/strings2011/video13

[106] N. Seiberg and E. Witten, Monopoles, duality and chiral symmetry breaking in $\mathcal{N}=2$ supersymmetric QCD, Nucl. Phys. B431 (1994), 484-550.

[107] S. Shadchin, On F-term contribution to effective action, JHEP 0708 (2007) 052.

[108] M. A. Shifman, Nonperturbative dynamics in supersymmetric gauge theories, Prog. Part. Nucl. Phys. 39 (1997), 1-116.

[109] M. Shifman and A. Yung, Non-Abelian Confinement in $\mathcal{N}=2$ Supersymmetric QCD: Duality and Kinks on Confining Strings, Phys. Rev. D81 (2010) 085009.

[110] V. P. Spiridonov, On the elliptic beta function, Uspekhi Mat. Nauk 56 (1) (2001), 181-182 (Russian Math. Surveys 56 (1) (2001), 185-186).

[111] V. P. Spiridonov, Theta hypergeometric integrals, Algebra i Analiz 15 (6) (2003), 161-215 (St. Petersburg Math. J. 15 (6) (2004), 929-967).

[112] V. P. Spiridonov, Elliptic hypergeometric functions, Habilitation Thesis (Dubna, 2004), 218 pp.

[113] V. P. Spiridonov, Elliptic hypergeometric functions and Calogero-Sutherland type models, Teor. Mat. Fiz, 150 (2) (2007), 311-324 (Theor. Math. Phys. 150 (2) (2007), 266-277).

[114] V. P. Spiridonov, Essays on the theory of elliptic hypergeometric functions, Uspekhi Mat. Nauk 63 (3) (2008), 3-72 (Russian Math. Surveys 63 (3) (2008), 405-472).

[115] V. P. Spiridonov, Elliptic beta integrals and solvable models of statistical mechanics, Contemp. Math. 563 (2012), 181-211, arXiv:1011.3798 [hep-th].

[116] V. P. Spiridonov and G. S. Vartanov, Superconformal indices for $\mathcal{N}=1$ theories with multiple duals, Nucl. Phys. B824 (2010), 192-216.

[117] V. P. Spiridonov and G. S. Vartanov, Elliptic hypergeometry of supersymmetric dualities, Commun. Math. Phys. 304 (2011), 797-874.

[118] V. P. Spiridonov and G. S. Vartanov, Supersymmetric dualities beyond the conformal window, Phys. Rev. Lett. 105 (2010) 061603.

[119] V. P. Spiridonov and G. S. Vartanov, Superconformal indices of $\mathcal{N}=4$ SYM field theories, Lett. Math. Phys. 100 (2012), 97-118. 
[120] V. P. Spiridonov and G. S. Vartanov, Elliptic hypergeometric integrals and 't Hooft anomaly matching conditions, JHEP 1206 (2012) 016.

[121] Y. Terashima and M. Yamazaki, $S L(2, \mathbb{R})$ Chern-Simons, Liouville, and Gauge Theory on Duality Walls, arXiv:1103.5748 [hep-th]; Semiclassical Analysis of the 3d/3d Relation, arXiv:1106.3066 [hep-th].

[122] J. Teschner, On the relation between quantum Liouville theory and the quantized Teichmüller spaces, Int. J. Mod. Phys. A19S2 (2004), 459-477.

[123] G. S. Vartanov, On the ISS model of dynamical SUSY breaking, Phys. Lett. B696 (2011), 288-290.

[124] B. Willett and I. Yaakov, $\mathcal{N}=2$ Dualities and $Z$ Extremization in Three Dimensions, arXiv:1104.0487 [hep-th].

[125] E. Witten, An SU(2) anomaly, Phys. Lett. B117 (1982), 324-328.

[126] Y. Yoshida, Localization of Vortex Partition Functions in $\mathcal{N}=(2,2)$ Super Yang-Mills theory, arXiv:1101.0872 [hep-th].

Bogoliubov Laboratory of Theoretical Physics, JinR, Dubna, Moscow Region 141980, RusSIA; E-MAIL ADDRESS: SPIRIDON@THEOR.JINR.RU

Max-Planck-Institut für Gravitationsphysik, Albert-Einstein-Institut 14476 Golm, Germany; e-mail address: vartanov@aei.mpg.de. Current address: DESY Theory, Notkestr. 85, 22603 Hamburg, Germany 